\title{
COMMUNICATIONS
}

\section{THE EYE IN GENERAL VASCULAR DISEASE* HYPERTENSION AND ARTERIOSCLEROSIS}

\author{
BY \\ ROBERT LEISHMAN \\ Department of Ophthalmology, University of Glasgow
}

THE HUMAN eye is a natural transparent chamber and its retinal vascular system is easily accessible to direct and repeated observations in vivo. The behaviour of its arterioles, capillaries, and venules in health and disease should provide data applicable to the study of systemic vascular disorders.

With the introduction of the ophthalmoscope, a little over a hundred years ago, abnormal fundus pictures were soon observed to occur in conditions of generalized disease, and, since then, many clinical observations and histological studies have been contributed to the ophthalmic literature. It is, however, a serious limitation of ophthalmoscopy that, even with the most modern instruments, a direct study of the retinal blood vessels at a cellular level is not possible, and it has proved most difficult to translate the descriptive terminology derived from ophthalmoscopic studies into terms of basic pathology.

It has been said, with perhaps some justification, that "specialism, though favourable to the accumulation of facts, is bad for the philosophy of knowledge". Most ophthalmologists would claim to recognize "copper-wire" and "silver-wire" arteries in the retina, but few would profess to have a clear conception of the meaning of these appearances in detailed terms of morbid histology. There is a danger that the faithful recording of clinical signs, such as "copper-wire" arteries, calibre variations, arterio-venous ratios, and the vagaries of the vascular light reflex, may become the self-imposed limit of legitimate clinical thought concerning the retinal vessels; and, having built up a series of uncommon attributes, it is easy to convey the implication that the retinal vessels are fundamentally different from other blood vessels.

In the field of general medicine, the invention of the sphygmomanometer in 1896 came at a time when physicians were preoccupied with arteriosclerosis, and, almost immediately, attention was diverted to a new factor in vascular disease, high blood pressure. This disorder has come to be known as hypertension; its study from many different aspects has led to great advances in the understanding of cardiovascular derangements. Abundant data are now available with regard to both hypertension and arteriosclerosis, but the data lack integration; it is still not generally appreciated that these disorders are to some extent complementary to one another, so that neither condition 
can be adequately assessed without considering the effect of the other. For this reason, physicians at the present time find it difficult to classify their cases of cardiovascular disease into groups which are clinically homogeneous; this is a major hindrance to the application of the new knowledge to the assessment and management of cases, and to the rational use of the many new hypotensive drugs which are now becoming available.

Since conventional ophthalmoscopic interpretations and descriptive ocular data do not appear to fit well with modern conceptions of related systemic diseases, it might be more profitable to reverse the method of approach. After consideration of the knowledge derived from research along general clinical, experimental, and pathological lines, a working hypothesis integrating hypertension with arteriosclerosis has been formulated, and this hypothesis has been tested in the ocular field.

\section{General Considerations}

The existence of arterial disease has been known for several centuries. The atheromatous lesion was observed first. Atheroma occurs in arteries of all sizes; it is a patchy, subendothelial, fatty, degenerative lesion with associated fibrosis. It occurs with, or without, high blood pressure, and its origin is not known.

Later, the name arteriosclerosis was coined to describe a diffuse hardening or stiffening of the arteries, contrasting with patchy atheroma. The common type is that which is due to high blood pressure. The initial response in the artery wall is physiological hypertonus; this is followed by hypertrophy and hyperplasia in the media, and if time is allowed, by replacement fibrosis, changes which are considered to be of a reactive nature.

It is to Allbutt (1896), that we attribute the recognition of high blood pressure as a disease entity with a course of its own. He segregated the condition during a clinical study of arteriosclerosis. His essential thesis, however, was not so much the recognition of hyperpiesia, but rather the differentiation of the decrescent from other types of arteriosclerosis. Decrescent or involutionary sclerosis is defined as an ageing process affecting arteries and arterioles, appearing histologically as a relative or replacement fibrosis of patchy distribution. The condition is not caused by, but may be associated with, high blood pressure.

It was natural, in the first instance, that arteriosclerosis and high blood pressure should be regarded as cause and effect, and, when this assumption was discredited, interest in arteriosclerosis waned. But, although arteriosclerosis does not produce high blood pressure, we have seen that continued hypertension may ultimately cause reactive sclerosis.

Hypertension is now considered to result from increased peripheral resistance, which in turn is due to generalized and widespread hypertonus. Tonus is a sustained physiological contraction and is not to be confused with spasm which implies a sudden violent contraction of limited duration. The main- 
tenance of sustained tonus is not associated with increased expenditure of energy, for the tissue, while in this condition, uses no more oxygen than when in the relaxed state, even though it may be exerting quite considerable tension (Lovatt Evans, 1941).

High diastolic pressures have been shown to cause necrotic lesions in the walls of arterioles, but arteriosclerosis is thought to provide some defence against the necrotizing effects of severe hypertension (Wilson and Byrom, 1939). This concept of defence by sclerosis is conceived in the limited sense that the reactive changes induced in a vessel wall as a result of hypertension appear to protect the wall from the more severe acute degenerative lesions of high blood pressure. The idea of "defence by sclerosis" may have a much wider application than this with regard to high blood pressure. Sclerosis may not only protect the wall from necrosis, but also, may in some cases predetermine, and in others modify, the whole course of the hypertensive disorder, a theme which we shall now proceed to develop more fully.

\section{HYPOTHESIS AND ARGUMENT}

If we accept Allbutt's differentiation of involutionary sclerosis as an ageing process in the arteries, independent of, but sometimes associated with, high blood pressure, we may divide the general population of non-hypertensive subjects into two groups in order to consider the effects of high blood pressure upon them:

(1) Those significantly affected by involutionary sclerosis.

(2) Those not affected.

Involutionary sclerosis is of almost universal occurrence after the sixth decade (Duke-Elder, 1940), and must therefore be regarded as a normal concomitant of old age. It may occur prematurely in younger persons, whereas in some elderly people it may have progressed little at an advanced age. It commonly affects the aorta and larger arteries, but may also be found in the arterioles. Fibrosis of the larger vessels will result in elevation of the systolic: pressure without corresponding elevation of the diastolic level, provided the heart muscle is sound and attempts to maintain the peripheral circúlation.

The ophthalmoscopic features of the retinal arterioles in senile involutionary sclerosis are well known(Fig. 1, overleaf). The arterioles appear relatively straight and diffusely narrow, with acute-angled branchings, and the colour of the blood column is of diminished intensity. It has been suggested that this appearance is due to contraction of maturing fibrous tissue in the walls of the vessels, but histologists have found no evidence of diffuse fibrosis or of fibrous thickening of the vessel walls. It is probable that the pale, straight, narrow retinal arterioles of normal old age, are merely a manifestation of lowered peripheral pressure resulting from relative rigidity of the larger arteries. Patchy relative fibrosis may well be present in such retinal arterioles, 


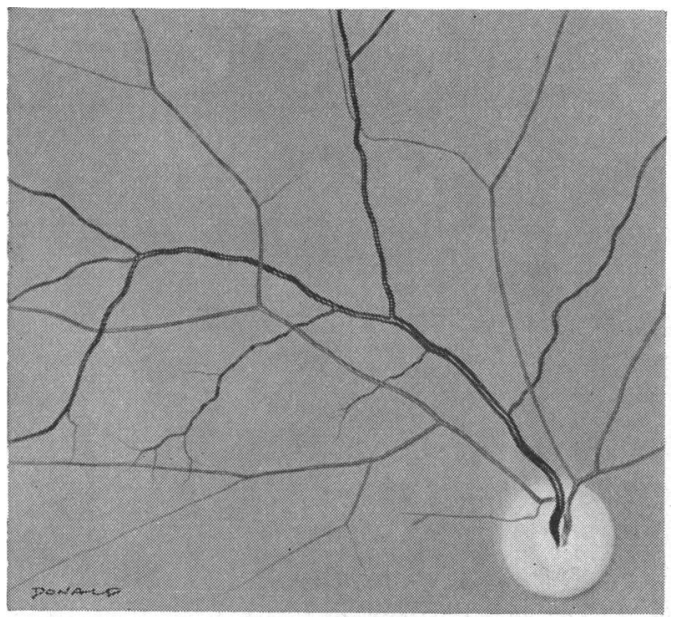

FIG. 1. - Diagram of senile retinal arteriole in a case of involutionary sclerosis without hypertension (blood pressure 225/85). The arterioles are diffusely straight and narrow, with acuteangled branchings, and the colour of the blood column is diminished.

but, since there is no increase of wall thickness and no deposition of abnormal elements, there should be no demonstrable alteration in the translucency of the arteriolar wall.

Hypertension, by definition, means a persistent elevation of both the systolic and diastolic levels of blood pressure. It is considered to result from the increased peripheral resistance offered by widespread diffuse arteriolar hypertonus. This conception assumes that the arterioles are capable of diffuse active constriction and takes no account of the state of the vessel walls with regard to fibrosis. Rigidity of the large arteries will tend to operate against elevation of the diastolic pressure, and patchy fibrosis of the arterioles will tend to reduce the area capable of effective hypertonus, so lessening the degree of increased peripheral resistance. Thus, if hypertension ensues in a person whose vessels are significantly affected by involutionary sclerosis, he will be protected from serious elevations of diastolic pressure in proportion to the degree and extent of his sclerosis.

Should hypertension occur in senile arterioles (Fig. 2), only those portions which are unaffected by fibrosis and still contractile, will become hypertonic; the blood column will become more narrow, and, since it is less bulky, will appear less red in colour when seen with the ophthalmoscope. In other portions of the wall, contractile elements will have been degraded into collagen fibres without actual increase in wall thickness. Such portions, with reduced contractility, will tend to dilate passively as a result of raised pressure; the blood column will become wider and more intense in hue, and elongation of the vessel wall will be revealed by slight localized curving of the wide portion of the column. In the course of time, as involutionary sclerosis advances, replacement fibrosis will extend into the hypertonic portions; the fibrotic segments will become confluent, and the whole vessel will then appear diffusely wide, red, and tortuous as a result of passive dilatation and elongation (Fig. 3, opposite). 


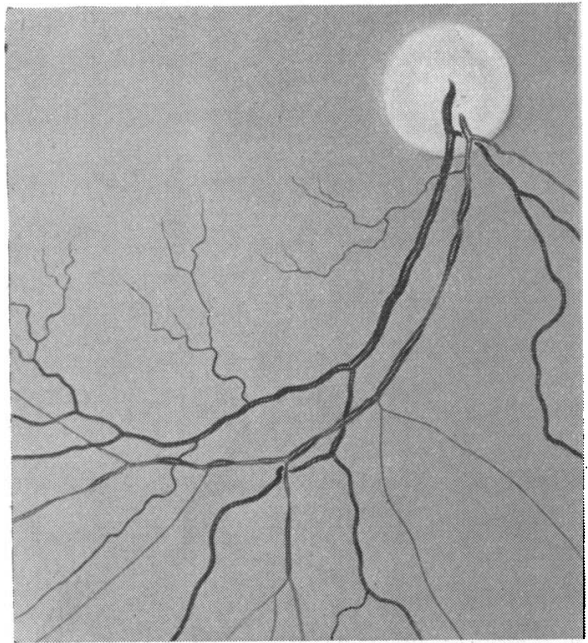

FIG. 2.-Diagram of senile retinal arteriole in a case of involutionary sclerosis with hypertension (blood pressure 200/120). Hypertonic portions of the vessel, where the wall is normal, are straight, narrow, and pale in colour. Other portions, where the wall is fibrous, are wide, deeper in colour, and more curvilinear.

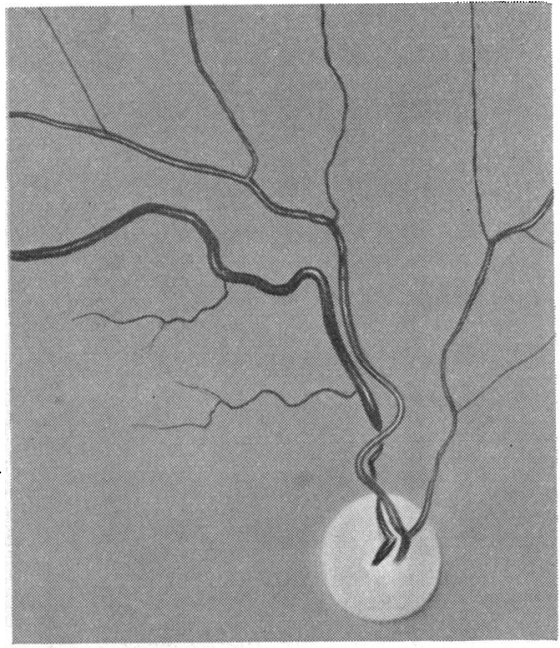

FIG. 3.-Diagram of senile retinal arteriole in a case of advanced involutionary sclerosis with hypertension (blood pressure 250/140). The main arteriole is diffusely wide, deep in colour, and tortuous.

If this reasoning is correct, aged retinal arteries which are usually pale, straight, and narrow in health, should become hypertonic in those parts of the arteriole which are still contractile; the blood column will remain pale, straight, and narrow in such portions, and the vessel wall should be of normal thickness and cell content in histological sections. Other segments which are fibrous, situated proximally, will become more red, wide and curvilinear; in these portions, with persistent rise of pressure, the walls may even become thinner than normal, and there should be a relative excess of collagen, while cell nuclei should be disproportionately few.

Since hypertonus is a physiological state, and involutionary sclerosis is a change which is on the border line between physiology and pathology, it is probable that neither condition will cause appreciable alteration in the translucency of the arteriolar wall. A vessel whose wall has undergone replacement fibrosis should become less elastic and more rigid, in the sense that it will offer greater resistance to stretching forces. But, when it is subjected to persistent abnormal pressure, it will tend to yield. In proportion to the degree of fibrosis, a vessel will lose the property of elastic recoil or resilience, and the more it is deficient in cellular elements, the more it will lose the power of active contraction.

We shall now consider the case of arteries not significantly affected by involutionary sclerosis. In the child or young person, age-fibrosis should be minimal. The normal child has well-coloured, wide, sinuous retinal 
arterioles (Fig. 4). Such vessels would be expected to respond actively and diffusely in hypertonus. Shortening of circularly and longitudinally disposed contractile elements would lead to changes in the appearance of the arteriolar blood column which would become straight, narrow, and more pale in colour in the hypertensive state (Fig. 5). The arteriole will react diffusely throughout its length, and the response will extend further in a proximal direction than in elderly subjects. The youthful resilience of the large arteries, together with the intensity and extensity of the peripheral reaction, will result in high levels of diastolic pressure. The state of hypertonus in youthful vessels in response to early hypertension is physiological in nature, and the vessel walls should appear normal in histological section.

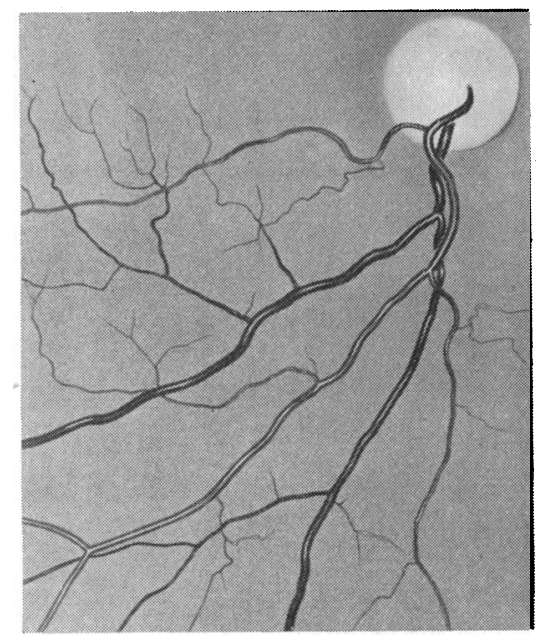

FIG. 4.-Diagram of normal young retinal arteriole in a non-hypertensive child. The arteriole is well-coloured, wide, and sinuous, with wide-angled branchings.

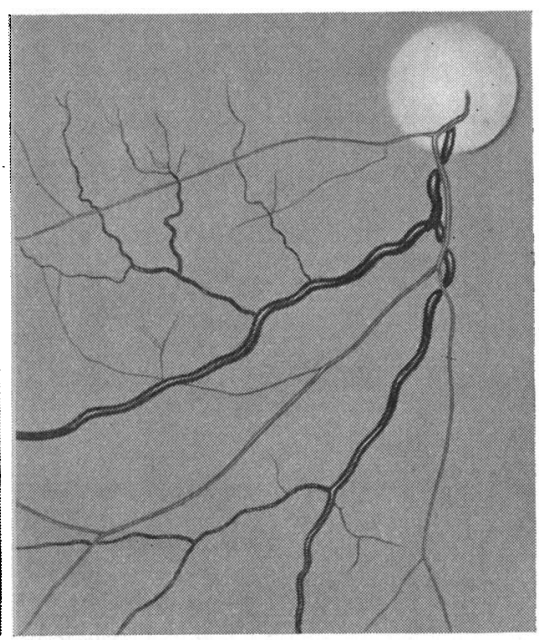

Fig. 5.-Diagram of a young retinal arteriole in a hypertensive child (blood pressure 160/100). The arteriole is straight, narrow, and more pale than normal, with acute-angled branchings, indicating diffuse hypertonus.

If the hypertensive stimulus is continued and is not too intense, reactive changes are stated to occur in the vessel wall, namely, hypertrophy and hyperplasia. Such changes are unlikely in walls already degraded by relative fibrosis, and should not be a prominent feature in the vessels of elderly hypertensive subjects. They might, however, develop more commonly in younger vessels, and by their nature, result in diffuse thickening of the arteriolar wall.

Ophthalmoscopically, a thickened wall containing many new-formed supporting elements should reflect more light and so dilute the colour of the blood column; it might also cause the arteriolar wall to become visible at an arterio-venous crossing. Hyperplasia occurs in the vessel wall while it is still hypertonic; so the blood column will remain narrow. Since formative cellular elements are irregularly placed in the wall, the blood column will 
also appear finely variable in calibre. Thus, a hyperplastic portion of an artery might appear clinically as a region which is excessively pale, with a blood column which is narrow and finely variable in calibre, and if such a portion were to cross a vein there would be concealment of the vein.

From this stage onwards, the further course of the arteriolar reaction will depend on the severity of the hypertensive stimulus.

In cases of fulminating hypertension, there will be no time for replacement fibrosis; hyperplasia will proceed to acute arteriolar necrosis.

In other cases, when the hypertensive stimulus is less severe, diffuse replacement fibrosis has time to supersede hyperplasia in the arteriole, diminishing its cellular content and hence its reactivity, and offering some protection against the necrotizing effects of high diastolic pressure. Since hypertonus merges into hyperplasia and in turn into fibrosis, all three processes may occur at the same time but in different portions of the same arteriole. Hypertonic branches will remain straight, narrow, and pale in colour; thick-walled hyperplastic portions will appear excessively pale and finely variable in calibre and will cause concealment at arterio-venous crossings; portions undergoing replacement fibrosis will be red, wide, and curvilinear (Fig. 6).

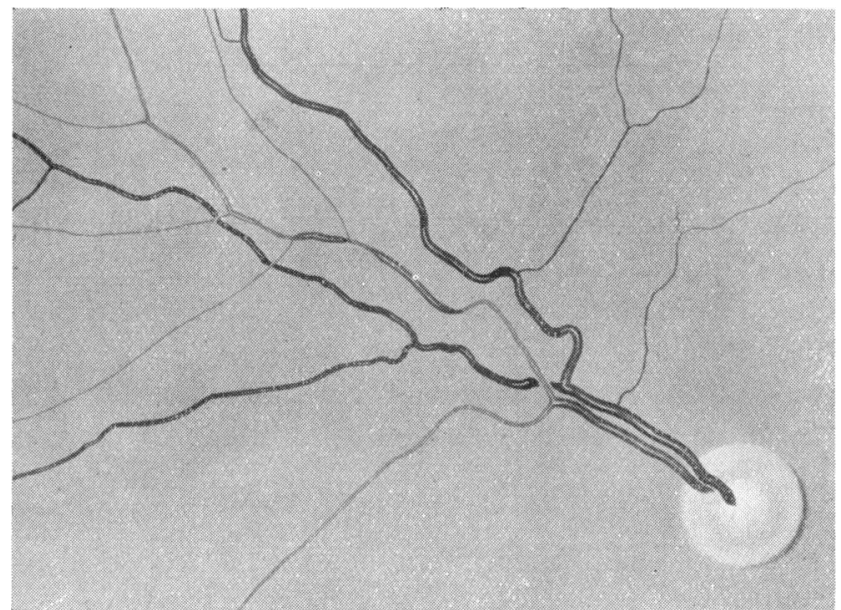

Fig.6.-Diagram of retinal arteriole in a case of hypertension with reactive sclerosis (blood pressure 250/130). Small branches are straight, narrow, and pale, indicating hypertonus; hyperplastic segments are excessively pale, finely variable in calibre, and cause concealment at arteriovenous crossings; fibrotic portions are wide and deep in colour.

If the patient survives until the stage of advanced reactive sclerosis is attained, a diffuse replacement fibrosis will have extended throughout the larger arterioles. The main arterioles will appear red, wide, and even tortuous throughout their entire length; minor branches, less capable of fibrosis, may still appear straight, narrow, and pale in colour, indicating hypertonus (Fig. 3). Thus the patient might reach a state of acquired benign hypertension resulting from reactive sclerosis, but differing in pathogenesis from benign hypertension which is predetermined by involutionary sclerosis.

From these data a hypothetical clinical classification has been constructed. This is summarized in Table I (overleaf). 


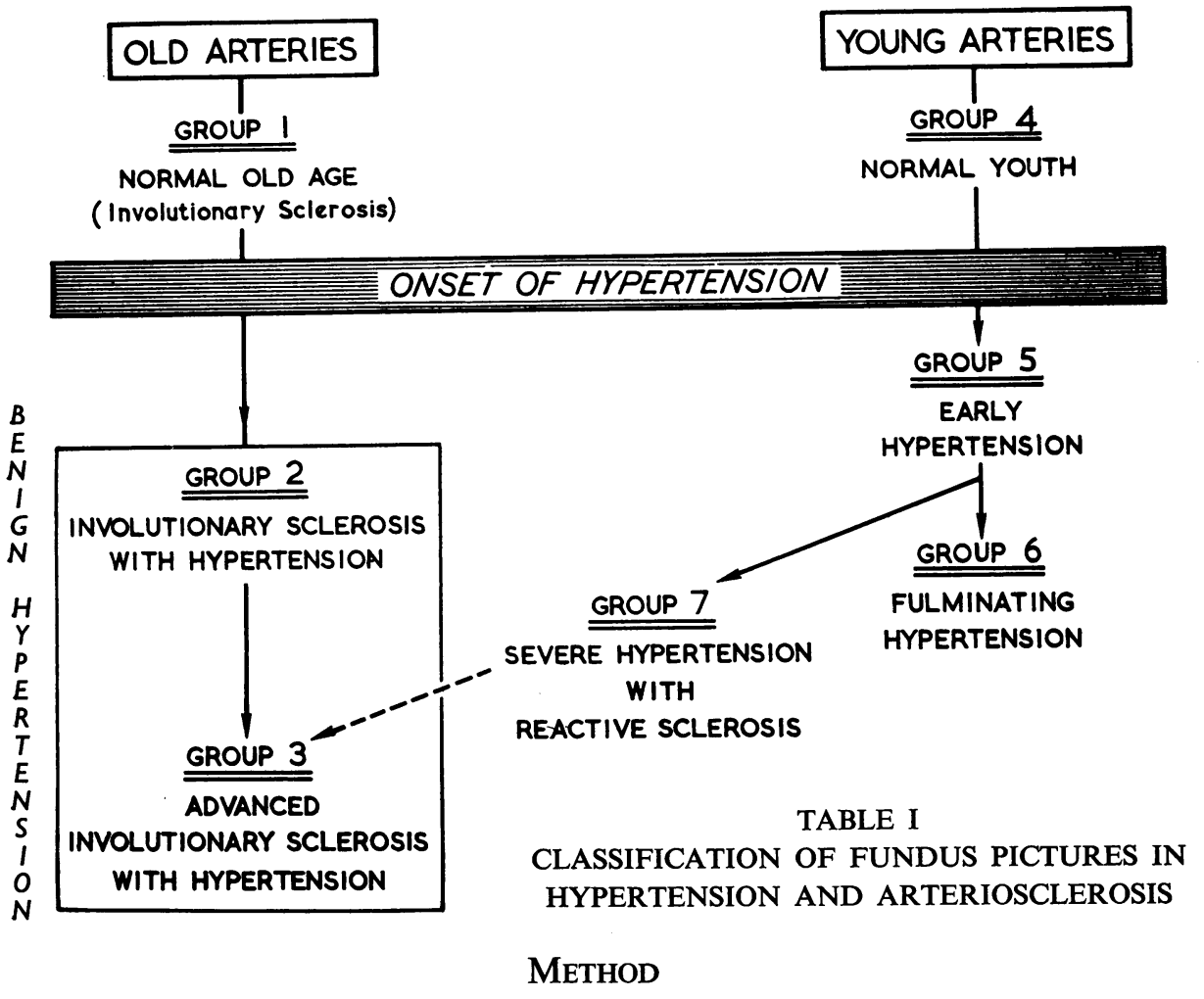

The phenomena provided by disease processes cannot be simplified artificially in order to fulfil the criteria of a good experiment, and the resulting observations must be pondered and interpreted to a point rarely necessary in a designed laboratory experiment.

The investigations to be described are mainly clinical; and it has proved extraordinarily difficult to make consecutive studies ante and post mortem in the same patient. Thus, in such studies, thought must travel ahead of pathological confirmation. On the other hand, a hypothesis which is based on physiological as well as pathological phenomena cannot be proved in all respects by pathological methods.

The steps to be taken in an ideal investigation of this type are as follows:

(1) Consecutive ophthalmoscopic studies with serial photographic records in life. These records of the fundus appearances were obtained by multiple exposures using a Zeiss-Nordensen camera. The photographic prints were fitted into each other to form a composite picture of the area of the fundus under observation.

(2) Photography of the fundus just before death.

(3) Early fixation of the eyes and removal of the posterior halves of the globes after death.

(4) Slit-lamp examination of the retina in situ. 
(5) Photography and microscopic examination of the retina in bulk after clearing in glycerine.

(6) Microscopic examination of serial sections of the retina, cut, quadrant by quadrant, in a direction at right angles to the course of the main vessels. Adjacent sections taken for study by different staining methods.

(7) Special localization and study of selected features.

Needless to say, the practical realization fell short of the ideal in most cases, but the above principles were adhered to in so far as was practicable.

\section{OphTHALMOSCOPIC GRADING}

The hypothesis was tested in the first instance by the method of clinical ophthalmoscopy.

The initial problem was two-fold. First, it was necessary to determine whether the existence of the hypertensive state could be recognized solely by ophthalmoscopic examination. This was found to be possible except in a very few cases, and the magnitude of error was well within the limits usually permitted in a clinical experiment. Secondly, it had to be discovered whether or not the various hypothetical fundus pictures could be segregated by means of the ophthalmoscope alone, without reference to other general medical data. The observed fundus pictures fell into seven groups as in Table I :

Group 1. Involutionary Sclerosis. - Since involutionary sclerosis is almost universal after 60 years of age, this represents the normal fundus in old age. In the elderly, the optic disc is usually pale and often slightly hollow like a shallow saucer. The ocular media become hazy; in the fundus background, reflexes are diminished, the hue is dull, retinal pigmentation is disturbed, and colloid bodies are usually present and often numerous. The retinal arteries run a relatively straight course, are diffusely narrow and slightly

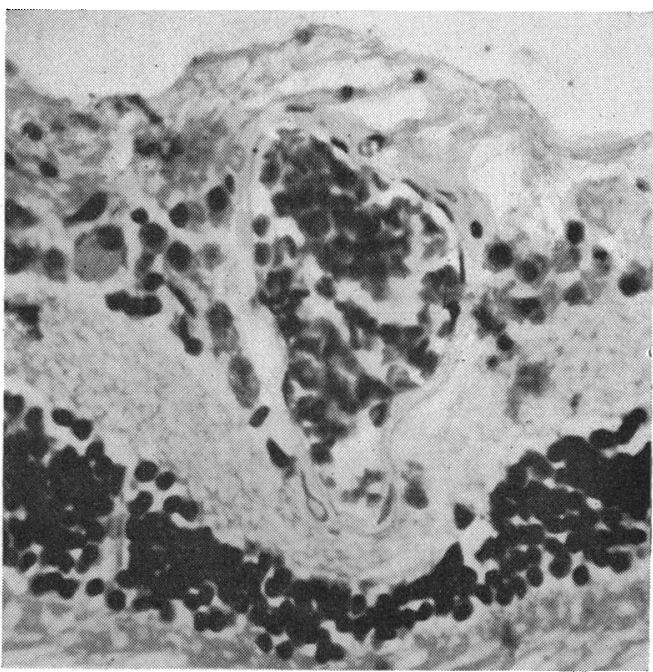

FIG. 7.-Section of normal retinal arteriole from a non-hypertensive elderly subject, showing a wall of diminished thickness with relative excess of collagen and scanty nuclei. $\times 400$. pale, and tend to branch at an acute angle; the walls, in health, are not visible. The veins are not congested distal to arterio-venous crossings.

The retinal arterioles of non-hypertensive, elderly subjects have walls of normal or diminished thickness, in which there is a relative excess of collagen and cell nuclei are disproportionately few (Fig. 7). 
This fundus picture is usually associated with raised systolic pressure without a corresponding rise in diastolic pressure, a condition which has been called systolic hypertension. Although these elderly persons usually enjoy good health for their years, the state of the general health and the blood pressure readings are consistent with extensive involutionary sclerosis, and the various features of the fundus picture are explicable as the manifestations of diminished peripheral pressure (Fig. 8).

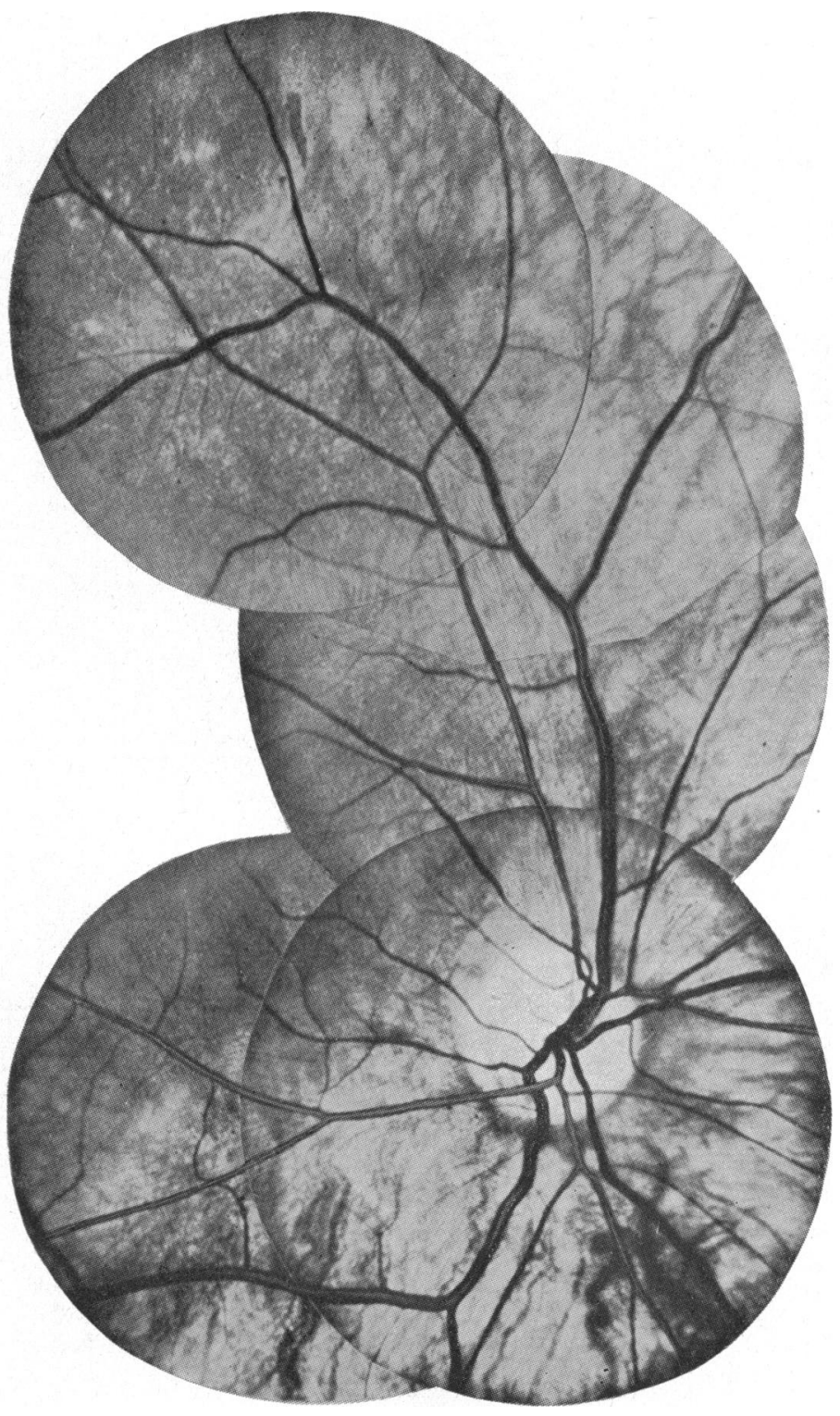

Fig. 8.-Fundus photograph in a case of involutionary sclerosis, showing a normal aged fundus (B.P. 225/85). The retinal arterioles are straight, diffusely narrow, slightly pale, and branch at acute angles, on an aged background. The veins are not congested distal to arterio - venous crossings. 
Group 2. Involutionary Sclerosis with Hypertension.-This type of picture seems to represent hypertension occurring in arterioles already partially defended by patchy sclerosis. Primary sclerosis may be the factor which predetermines the benign course of the hypertensive disorder in this group of cases.

Certain fundi, while presenting all the features of senility in the fundus background, show modifications in the arteriolar picture which differ strik-

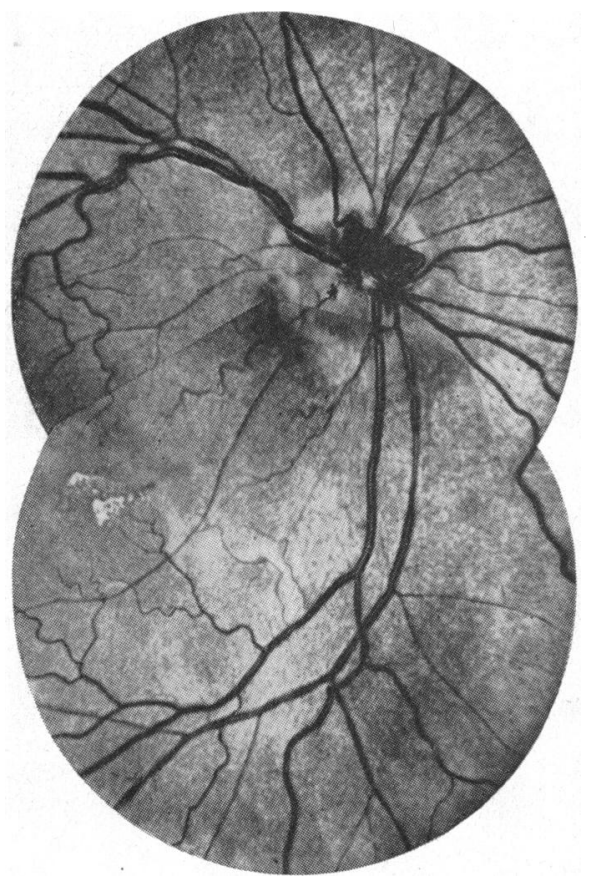

FIG. 9.-Fundus photograph in a case of involutionary sclerosis with hypertension (blood pressure 250/120). Hypertonic portions of the vessel, where the wall is normal, are straight, narrow, and pale in colour. Other portions, where the wall is fibrous, are wide, deep in colour, and more curvilinear. The veins are congested distal to arterio-venous crossings. The background shows senile changes. ingly from the expected appearance of the vessels in normal old age (Fig. 9).

Small retinal arterioles, and the distal portions of the larger arterioles, remain straight and narrow, but if one of the main retinal arteries is followed from the periphery towards the disc, it suddenly becomes more red, wide, and curvilinear, and, in this dilated portion, bears a superficial resemblance to a normal vessel in a younger age group. In the region from the midperiphery to the disc, segments of unduly wide calibre are separated by other segments which are of expected narrow calibre. There is no concealment of the vein at arterio - venous crossings. The portion of the vein distal to some of the crossings shows more intense colour, with increased calibre and tortuosity, features which indicate congestion of the vein.

The pathological picture is illustrated by the following case:

Case 1, benign hypertension, in a male, aged 68. 3 years before death, angina of effort B.P. $160 / 102$.

Cerebral haemorrhage, B.P. 250/105.

Died 5 hours after admission to hospital. General and histological post-mortem findings confirmed the clinical diagnosis of benign hypertension.

The fundus background is shown in Fig. 10 (overleaf). The retina and choroid together were cleared in glycerine and examined in bulk. The aged fundus shows a generalized disturbance of retinal pigment and a great excess of colloid bodies. The walls of the retinal arteries are not thickened in benign hypertension. Portions of the vessel have thin collagenous walls with absence of elastic fibres 
and with scanty nuclei (Fig. 11); elsewhere the wall is normal in structure (Fig. 12).

FIG. 10.-Case 1, flat preparation of retina and choroid showing an excess of colloid bodies and generalized disturbance of retinal pigment. $\times 100$.

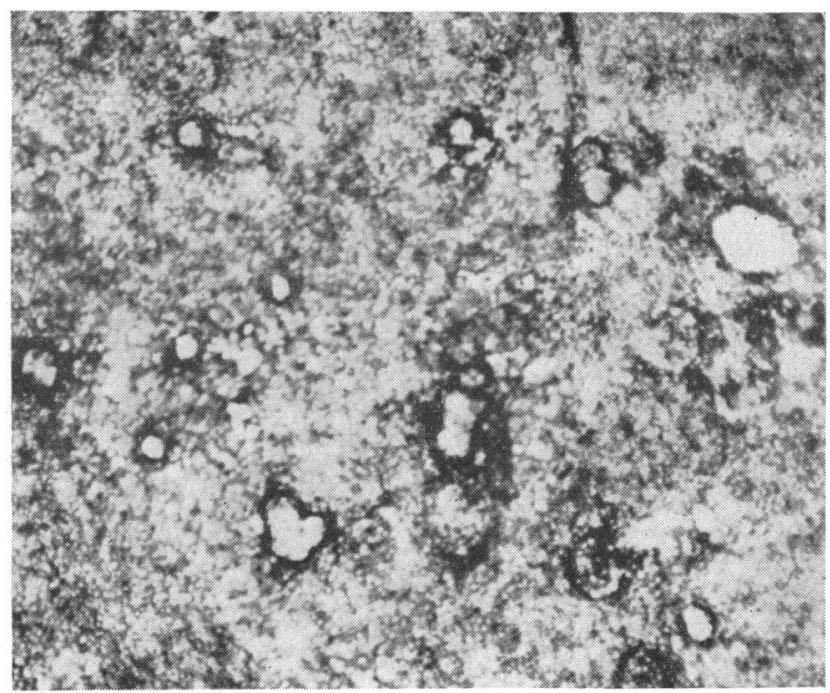

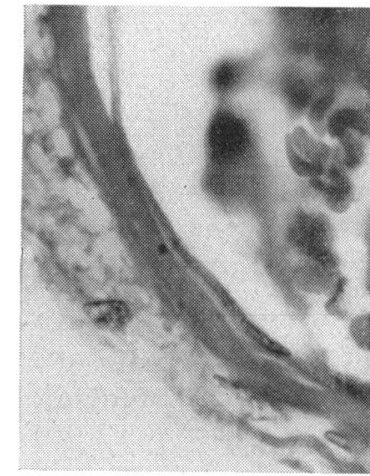

Haemalum and eosin

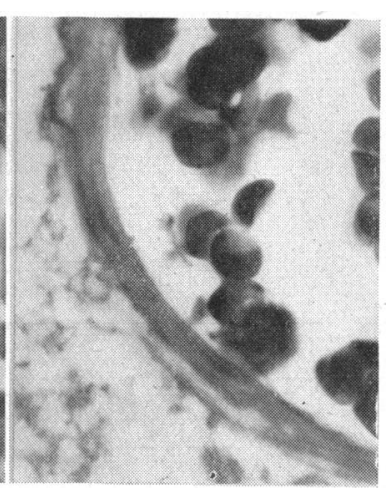

FIG. 11.-Case 1, adjacent sections from fibrous portion of arteriolar wall in involutionary sclerosis with hypertension. The walls are thin and collagenous with scanty nuclei and absence of elastic fibres. $\times 600$.

FIG. 12.-Case 1, section of normal portion of arteriole in involutionary sclerosis with hypertension. The wall shows normal thickness and cell content. $\times 400$.

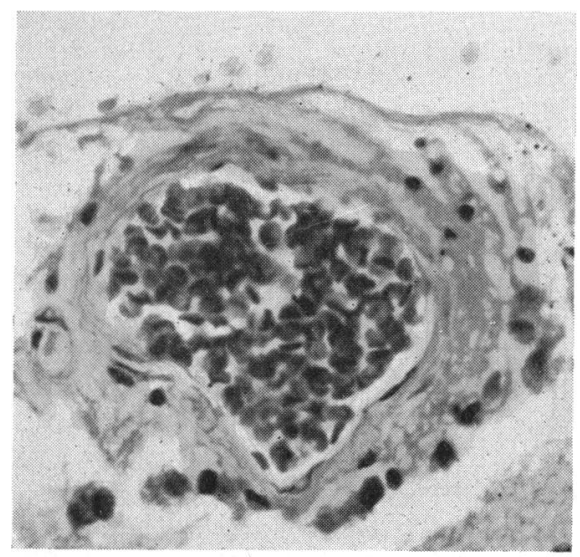


Where the wall is thin and fibrous, the lumen is wide and circular (Fig 13); such wide fibrous segments may be separated by narrower parts in which the walls have normal structure. In portions with relative fibrosis, cells are infrequent, and the wall consists mainly of collagenous fibres and may show slight hyaline change.
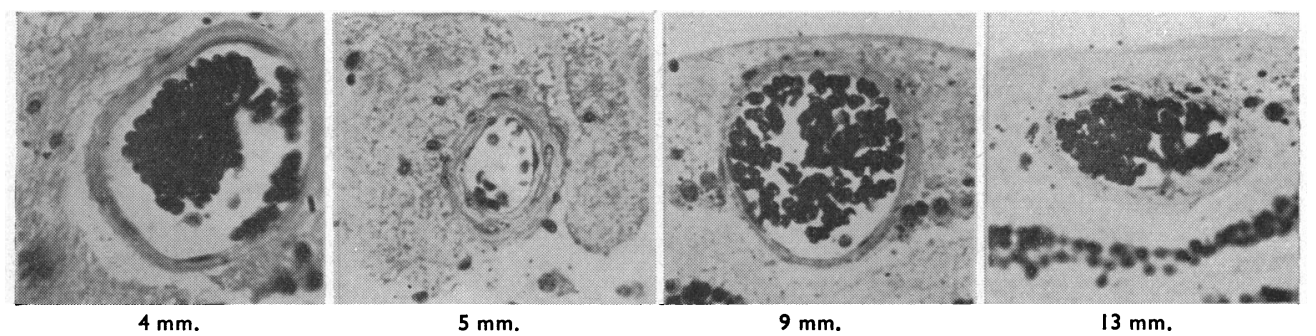

FIG. 13.-Case 1, serial sections of retinal arteriole in involutionary sclerosis with hypertension. Distance from optic disc shown in millimetres. Where the wall is thin and fibrous, the lumen is wide; where it is normal in structure, the lumen is narrow. $\times 250$.

The capillaries appear as small, circular, patent tubes of uniform internal diameter just wider than a red blood corpuscle. There is no swelling of the capillary endothelium (Fig. 14).

FIG. 14. Case 1, section of retina showing capillary bed in involutionary sclerosis with hypertension. The capillaries are patent and of uniform internal diameter just wider than a red blood corpuscle. There is no swelling of the endothelium. $\times 167$.

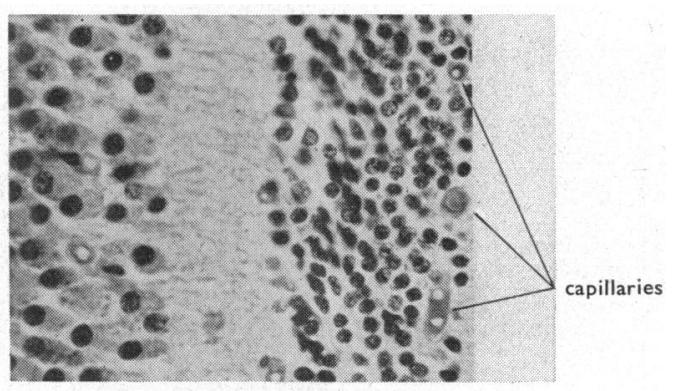

These findings are in accord with the hypothesis that hypertension has occurred in relatively fibrous vessels. Reactive change is absent, and the result of increased pressure is passive stretching of the wall in the regions of relative fibrosis.

These vascular features are to be found in elderly hypertensive subjects, or in younger subjects who show manifestations of premature senility. The majority of patients with high blood pressure seen in the ophthalmic outpatients clinic of a general hospital fall into this group. They are often symptom-free, and the condition is recognized in the course of routine fundus examination; sometimes they complain of headaches and giddiness and think they require a change of glasses. Several cases have presented on account of diplopia, with paresis of one or more of the extra-ocular muscles.

The systolic blood pressure is usually $\pm 200 \mathrm{~mm}$. $\mathrm{Hg}$, and the diastolic pressure ranges from 95 to $120 \mathrm{~mm}$. Hg. Thus the patient is hypertensive; and the combination of high systolic and moderately raised diastolic pressure suggests that there is a degree of age-fibrosis. The fundus appearances show that there are large segments of fibrosis in the retinal arterioles limiting the 
extensity of the hypertonic response. At certain of the arterio-venous crossings, where the anatomical disposition permits, a tense artery appears to cause some congestion throughout the distal distribution of the underlying vein.

With the passage of time, the arterioles in these cases show an extension of replacement fibrosis into hypertonic portions of the wall. These narrowed portions then appear to widen in calibre, with increase in colour intensity, until, finally, the main stem of the arteriole appears red, diffusely wide, and sinuous. This is well shown in fundus photographs of the same patient taken at an interval of 4 years (Fig. 15).

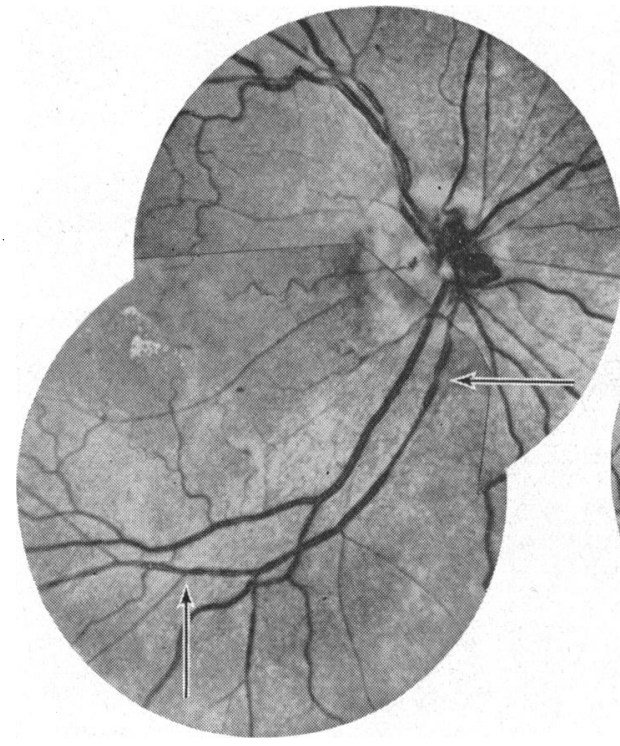

(a) February, 1953.

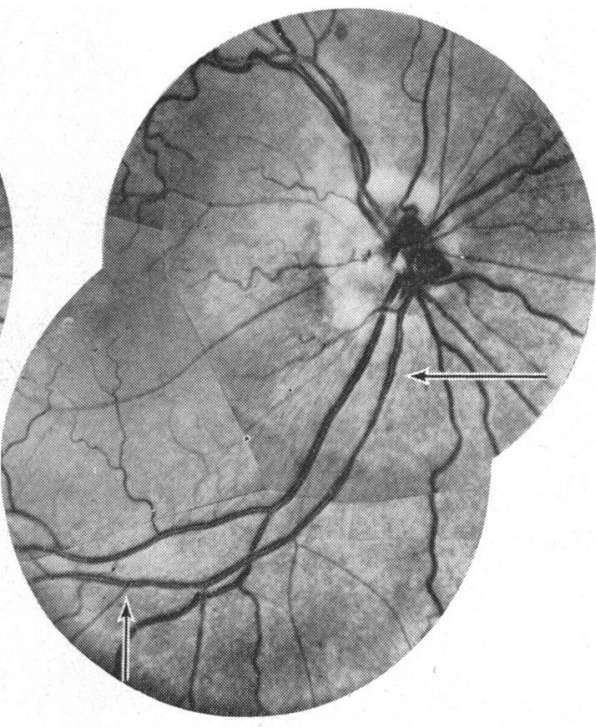

(b) March, 1957

FIG. 15.-Case 1, fundus photographs at an interval of 4 years. Narrowed portions of the arteriole have become wider in calibre with extension of fibrosis into these hypertonic segments.

Group 3. Advanced Involutionary Sclerosis with Hypertension.-The fundus background presents senile stigmata. The main retinal arterioles are unexpectedly red, wide, and tortuous, from the disc well out to the periphery, and there are no marked calibre variations in the main stem. In a superficial way, these vessels resemble the healthy vessels of a much younger age group, but they are out of place when viewed against an aged fundus background. Branches of the second and third orders are relatively pale, straight, and narrow (Fig. 16, opposite). There is no concealment of the vein at arterio-venous crossings, but congestion of the vein is present distal to some of these crossings. Nocase in this group has so far been examined histologically.

This type of picture is found in association with blood pressure levels of a higher order than those seen in Group 2. The patient may have no complaints, and may be unaware of his hypertension, although the diastolic 
pressure may reach $140 \mathrm{~mm}$. $\mathrm{Hg}$. The hypertensive disorder is usually of considerable standing in these cases, but the high levels of diastolic pressure seem to be well tolerated in vessels which are extensively fibrotic.

Group 4. The Normal Fundus in Youth.In youth (Fig. 17), the ocular media are clear, and the reflexes are brilliant. The optic disc is pale in young children, but it has a healthy pink colour in young adults. The fundus background has a glittering quality and a rich hue, retinal pigmentation is uniform, and there is an absence of colloid degeneration. Against this youthful background, the retinal arteries are wide in calibre and rich in hue. They course in wide sinuous curves over the fundus, branching at moderately wide angles, and have walls which are not visible. Histological section of a normal retinal arteriole from a young child shows that the proportion of cells to fibres is high (Fig. 18). This youthful picture is associated with normal blood pressure readings.

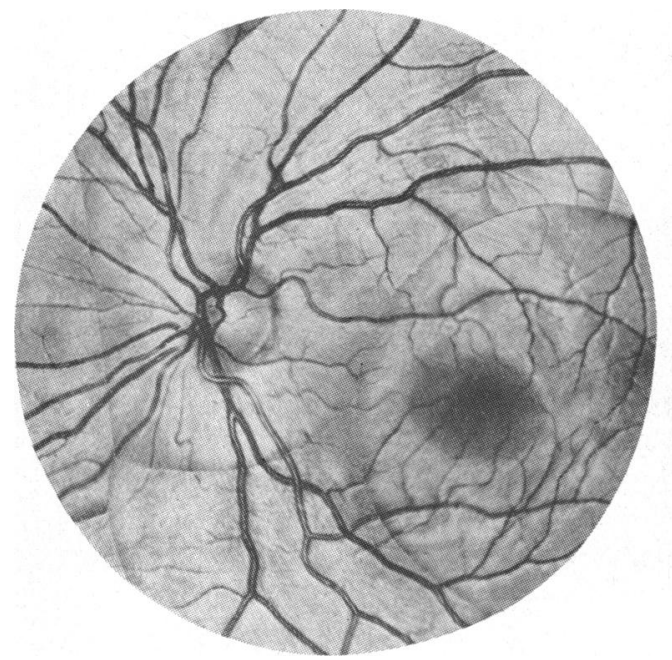

FIG. 17.-Fundus photograph in a normal youth. The retinal arterioles are wide, well-coloured, and sinuous. The fundus background has a glittering quality and the retinal pigmentation is uniform.

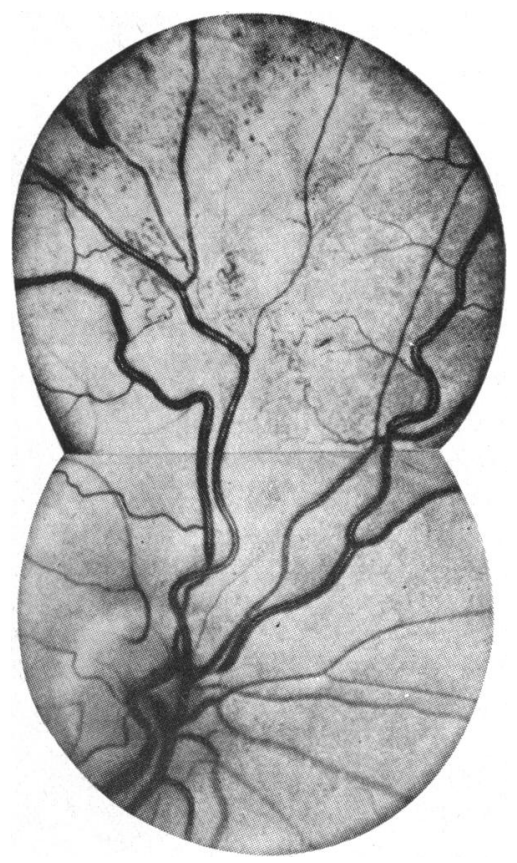

FIG. 16.-Fundus photograph in a case of advanced involutionary sclerosis with hypertension. The main arteriole is diffusely wide, deep in colour, and tortuous, while minor branches are relatively pale, straight, and narrow. Congestion of the veins is well-marked distal to arteriovenous crossings, but there is no concealment of the vein.

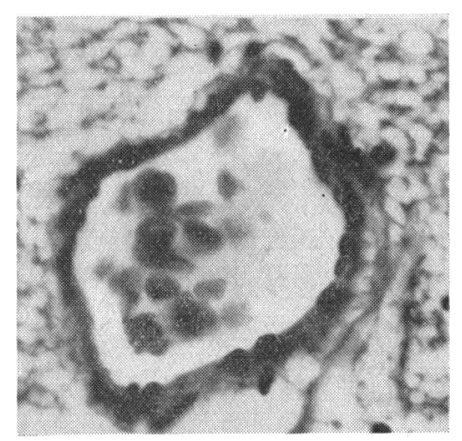

FIG. 18.-Section of normal retinal arteriole in a non-hypertensive child. The proportion of cells to fibres in the arteriolar wall is high. $\times 300$. 
Group 5. Early Hypertension in Youthful Vessels.-This type of picture represents diffuse hypertonus, the initial response of youthful vessels in the hypertensive state. Transient hypertension, which subsides rapidly with rest, is often, if not always, associated with acute glomerulo-nephritis in children. The ophthalmoscopic appearance of the retinal arteries was observed during and after the hypertensive phase. Not all cases of acute glomerulo-nephritis were hypertensive when first seen, but, when high blood pressure was present, it could be distinguished in the fundus by diffuse constriction of the retinal arterioles, which appeared unduly straight and narrow, with a blood column of diminished colour intensity (Fig. 19, opposite).

Congestion of the veins distal to some of the arterio-venous crossings was observed, but in no case of transient hypertension in children was there concealment of the vein at such a crossing. Repeated observation over a period of days or weeks in these cases showed that the arterioles regained normal colour, width, and sinuosity, after the hypertensive phase had passed.

Similar fundus appearances are not uncommon in young adults, and occur, for example, in hypertensive cases of acute glomerulo-nephritis and toxaemia of pregnancy (Fig. 20, opposite).

Arterioles which are well-coloured, wide, and sinuous in health, become straight, diffusely narrow, and less intense in colour, and are related to a youthful fundus background in the hypertensive state. There is no concealment of the veins at arterio-venous crossings, but congestion of the vein is present distal to some crossings.

Thus, the initial response to hypertension in the case of youthful vessels agrees with the expected picture of diffuse hypertonus. This arteriolar picture of hypertension in young vessels closely resembles the vascular picture in healthy elderly persons with involutionary sclerosis but without hypertension. In young people, arterioles which are pale, straight, and narrow, are abnormal against a youthful fundus background, and congestion of the distal portions of the veins will be observed at one or more of the arteriovenous crossings. Tension alone, of short duration, does not appear to reduce the translucency of the arteriolar wall.

Histological investigations have not been possible in this group.

Group 6. Fulminating Hypertension.-This type of picture is usually called " malignant " hypertension; the term " fulminating " is to be preferred. It need not be construed as a separate type of hypertension, but rather as an acutely progressive disorder resulting from a very severe hypertensive stimulus acting upon relatively youthful arterioles which are undefended by fibrosis.

Although it is probable that a phase of simple hypertonus occurs as a brief prelude to the development of fulminating hypertension in the retina, this transition has not yet been observed. 


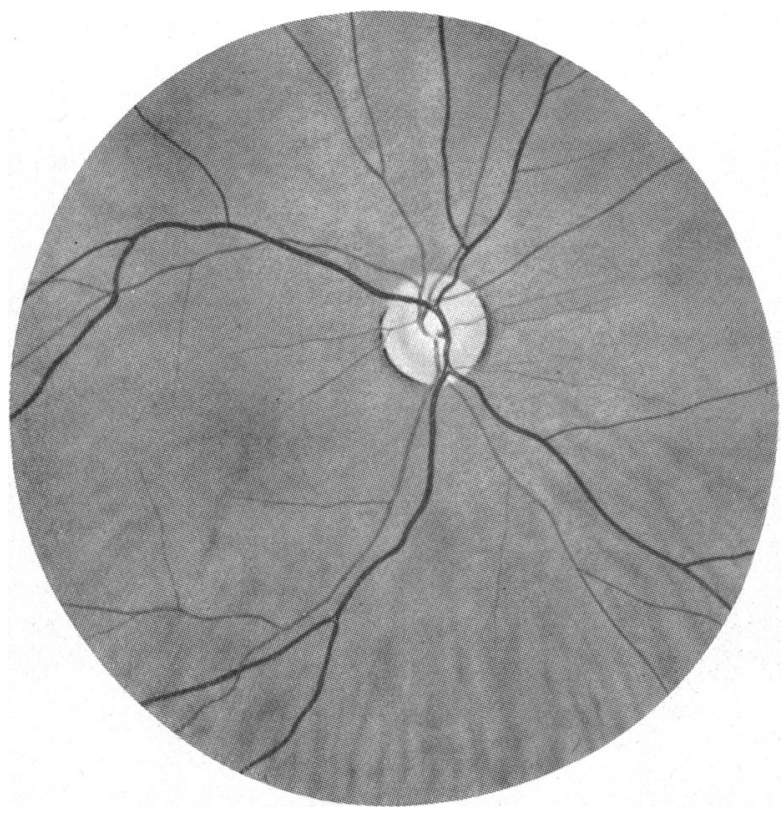

Fig. 19.-Drawing of fundus in transient hypertension in a child. The retinal arterioles are diffusely narrow, straight, and pale in colour. There is congestion distal to some of the arteriovenous crossings, but no concealment of the vein.

Fig. 20.-Drawing of fundus in early hypertension in a young adult. The retinal arterioles are diffusely narrow, straight, and pale in colour. There is congestion at some of the arterio-venous crossings, but no concealment of the vein.

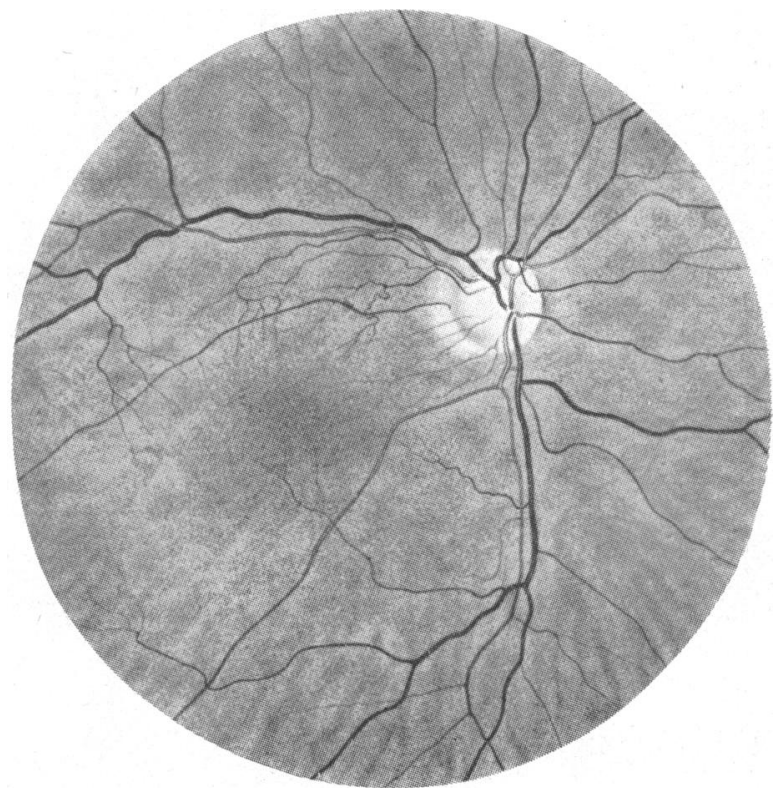


In fulminating cases (Fig. 21), papilloedema and retinopathy are usually present when the fundus is first examined. The papilloedema is always well marked and is often associated with oedema of the retina. Although the arteries may approximate to normal calibre near the disc, they are always diffusely pale. In some portions the pallor is accentuated and the calibre of

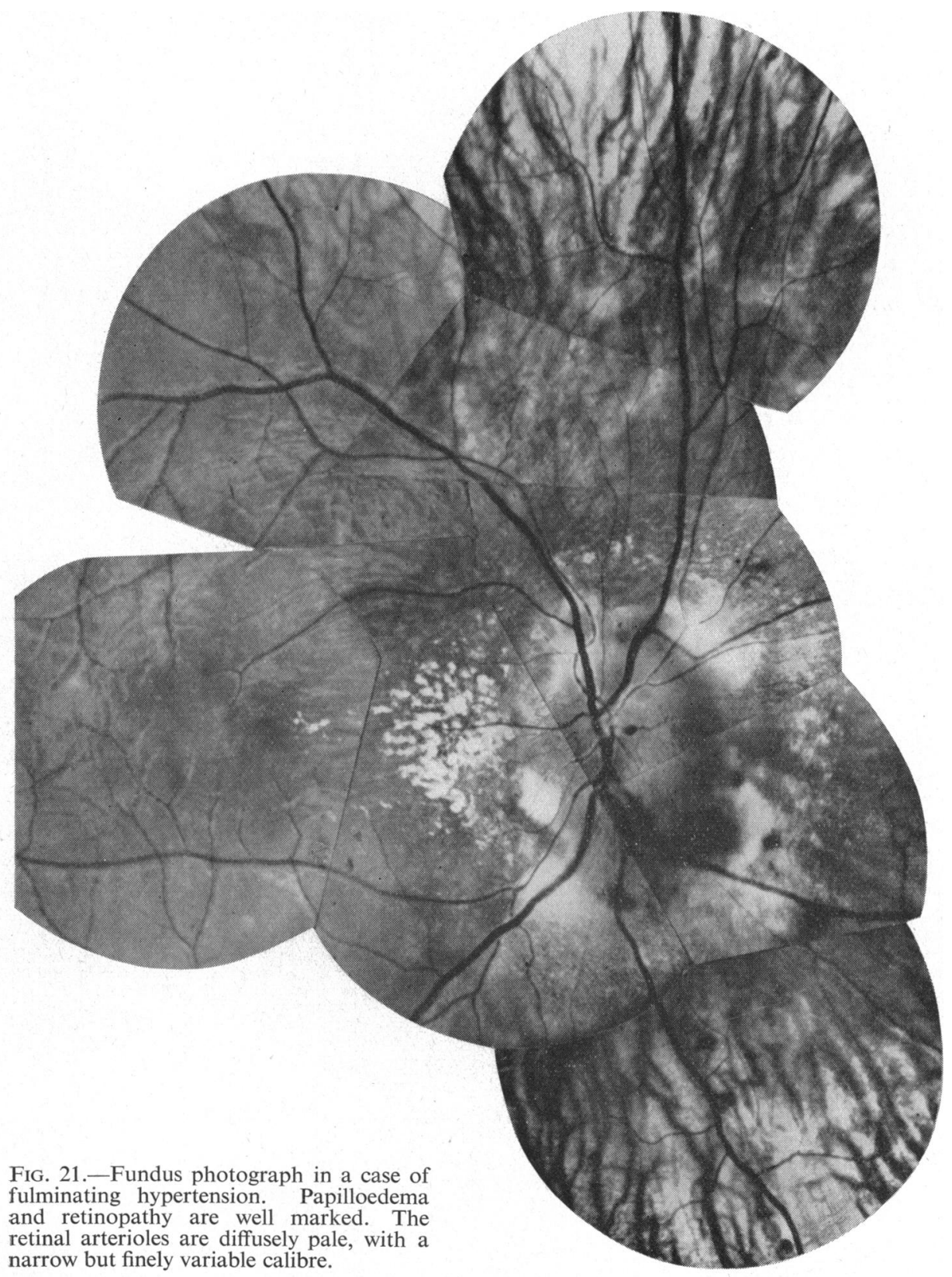


the blood column is reduced and finely variable. Broad parallel zones of concealment of the vein are seen at many of the arterio-venous crossings and are more marked if that segment of the artery shows increased pallor. These appearances suggest arteriolar walls which are thickened, but not necessarily rigid. They will be shown to be associated with hyperplasia in the vessel walls. The thickened arteries near the disc sometimes appear to yield to the lateral pressure, the blood column expanding diffusely in width but remaining diluted in colour; in the greater part, however, the arteries remain excessively pale, diffusely narrow, but finely variable in calibre, and become tortuous in three dimensions suggesting elongation; small vessels may be straight and narrow, suggesting hypertonus.

Small, round, hard-edged, white exudates are usually numerous around the disc and sometimes in the central area of the fundus. Superficial patches of "cotton-wool" exudate may be scattered here and there over the fundus, and such patches usually lie in close relation to a small arteriole. The clinical course of both types of exudate will be discussed later. Clusters of small haemorrhages which appear to have a capillary origin may be present in the fundus, and occasionally a larger haemorrhage may be seen radiating from a small straight arteriole with a thrombotic blood column.

Arteriolar thrombosis occurs in severe cases of hypertension and can be recognized with the ophthalmoscope (Fig. 22). A mass of haemorrhage appears around a retinal arteriole. The vessel is invisible distal to the haemorrhage but the proximal part is nearly filled with blood which is of deeper colour than arterial blood. The thrombotic blood column is straight and extends proximally almost to the parent artery, or, in other cases, to the nearest branch or branches, which are also thrombotic. The vessel then continues as a fine white thread until it joins the parent artery. In the course

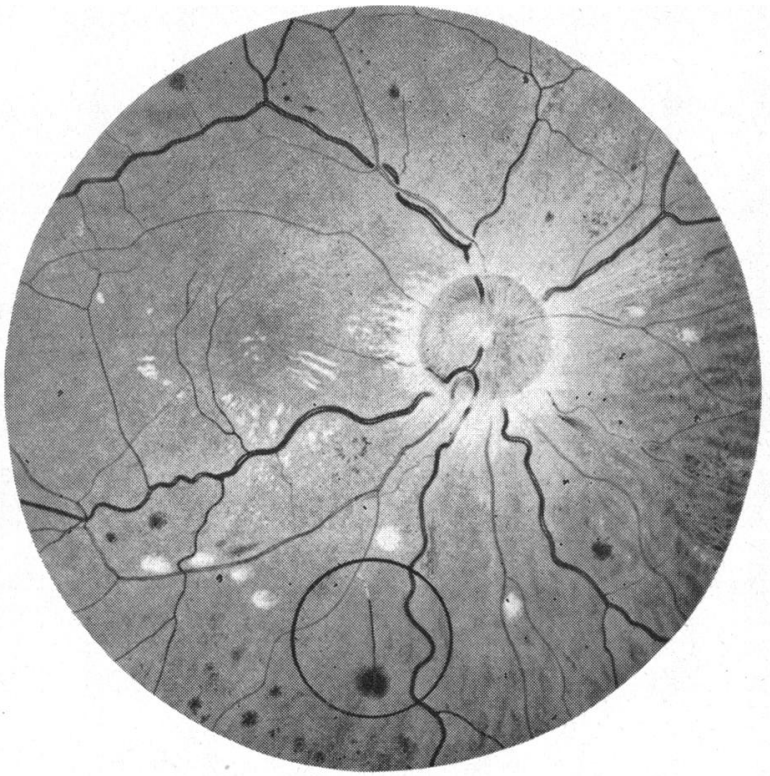

of a week or two the haemorrhage occupies a slightly larger area and appears less dense, and the blood vessel may be seen within it as a white ribbon;

Fig. 22.-Drawing of fundus in a case of fulminating hypertension showing arteriolar thrombosis. A haemorrhage radiates from a small arteriole (ringed). Distal to the haemorrhage the vessel is invisible. Proximal to the haemorrhage the blood column is straight, and deeper in colour than arterial blood; it is joined to the parent vessel by a thin white thread. 
the thrombotic blood column gradually disappears, the distal portion first. The white thread at the origin of the vessel persists indefinitely and may ultimately remain as the sole indication that there has been a thrombotic arteriole in this situation (Fig. 23).

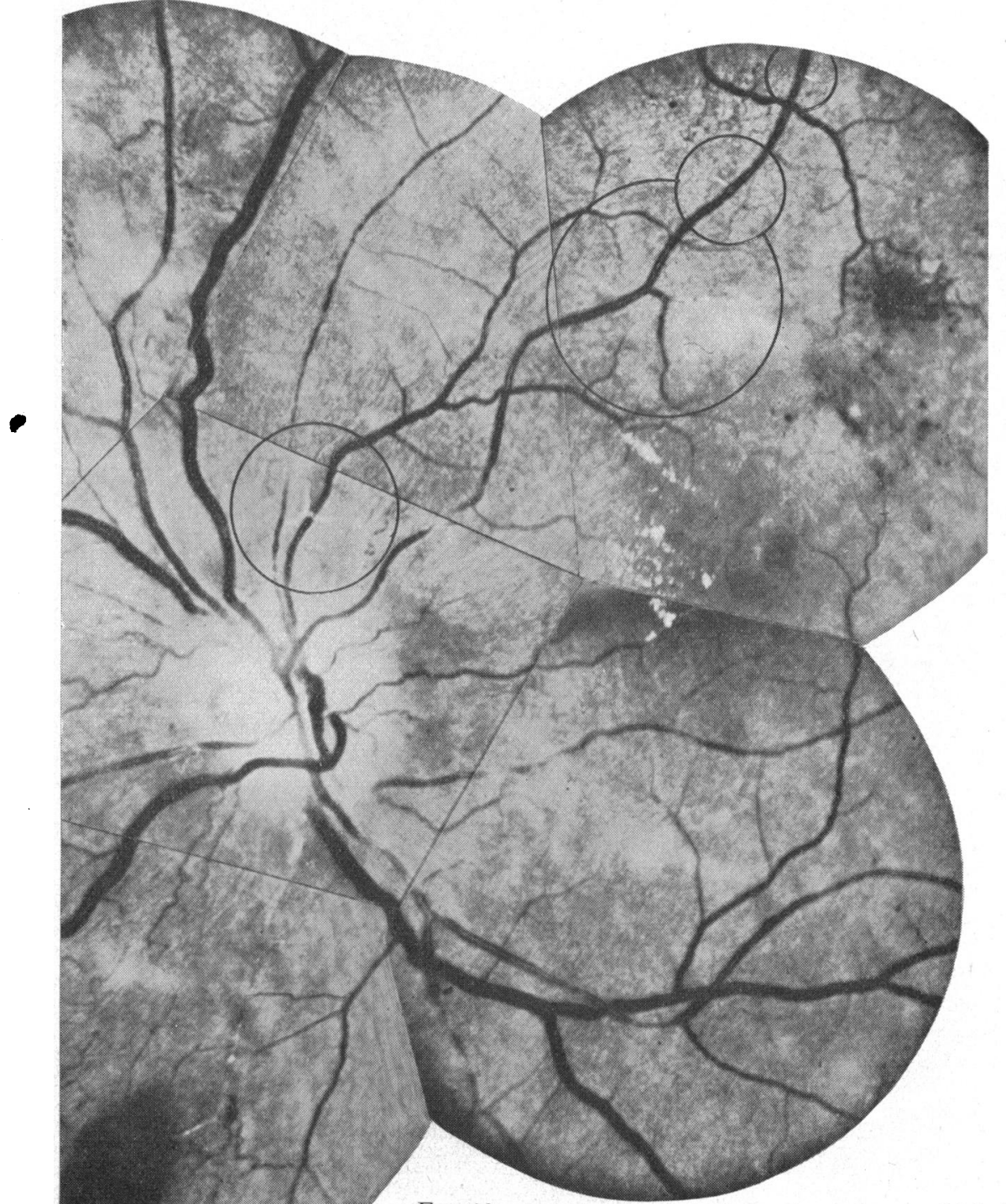

FIG. 23.-Fundus photograph in a treated case of fulminating hypertension; necrotic arteriolar remnants are seen as fine white threads (ringed).

This picture probably results from focal necrosis in the arteriolar wall, and may well be associated with similar lesions in the vessels of other organs. It has been seen in cases of severe hypertension with reactive sclerosis as well as in fulminating cases. 
The pathological features of fulminating hypertension are illustrated by the following case:

Case 2, fulminating hypertension, spinster, aged 50 years. Course of illness, 2 years; 5 months before death, B.P. $260 / 150$.

A few hours before death, B.P. 260/170. Massive pericardial effusion, heart failure. General and histological post-mortem findings confirmed the clinical diagnosis of fulminating hypertension.

The youthful nature of the fundus background is confirmed by the absence of colloid bodies in the choroid and retina (Fig. 24).

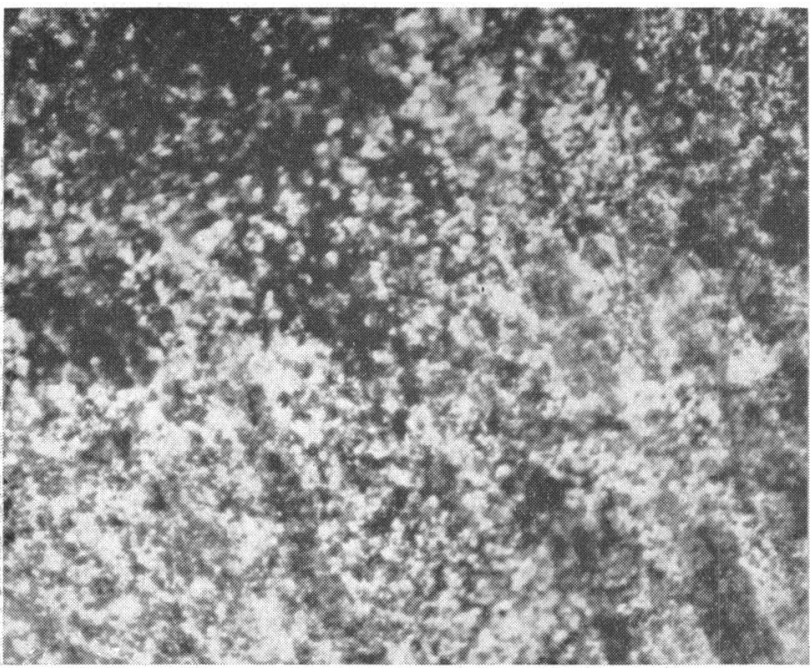

FIG. 24.-Case 2, flat preparation of retina and choroid, showing absence of colloid bodies. $\times 100$.

The walls of the arteries and arterioles are diffusely thickened; the lumen is diffusely narrow, and finely variable (Fig. 25).

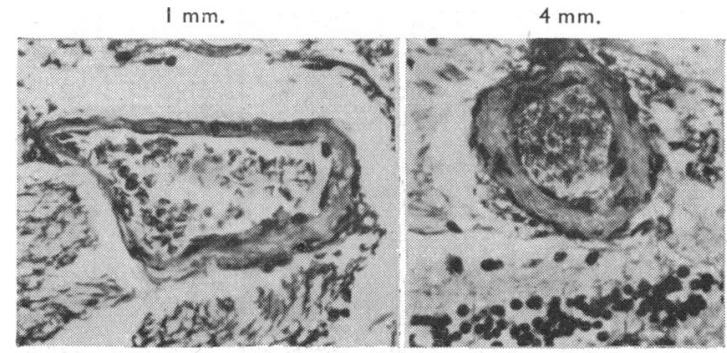

FIG. 25.-Case 2, serial sections of retinal arteriole in fulminating hypertension. Distance from optic disc shown in millimetres. The wall of the arteriole is diffusely thick; the lumen is diffusely narrow but finely variable. $\times 250$.

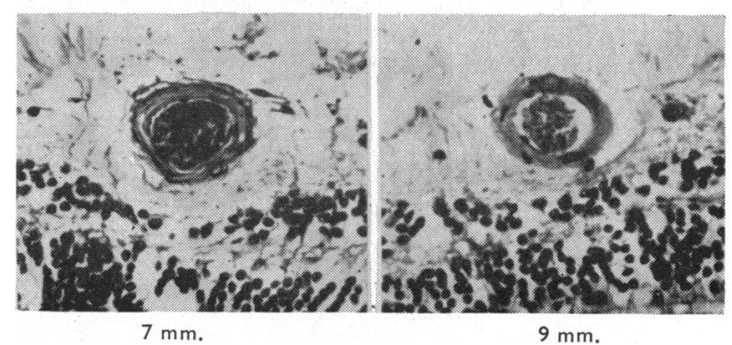


The thickening of the wall is partly due to swelling of the supporting fibres, but there is also evidence of hyperplasia, an excessive number of elastic fibres being present at all levels in the thickened wall (Fig. 26). Cells are not excessively numerous but are often swollen and faintly stained, and occasionally the nuclei are fragmented. The cell changes and hyperplasia are most marked where the swelling is greatest, and there the lumen is most narrowed. No proliferation of endothelium has been observed in the arteries or arterioles. The capillaries are usually widely dilated, although the calibre varies, and the endothelial cells are often greatly swollen (Fig. 27).

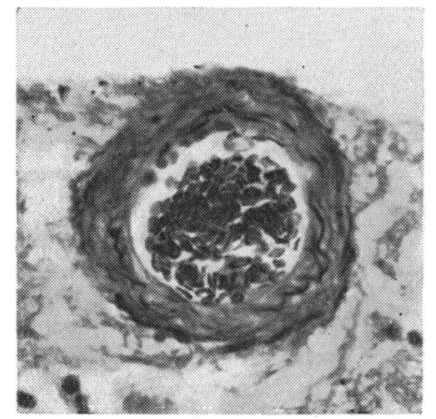

FIG. 26.-Case 2, section of retinal arteriole $6 \mathrm{~mm}$. from the optic disc in fulminating hypertension. Elastic fibres are present at all levels in the thickened wall. $\times 200$.

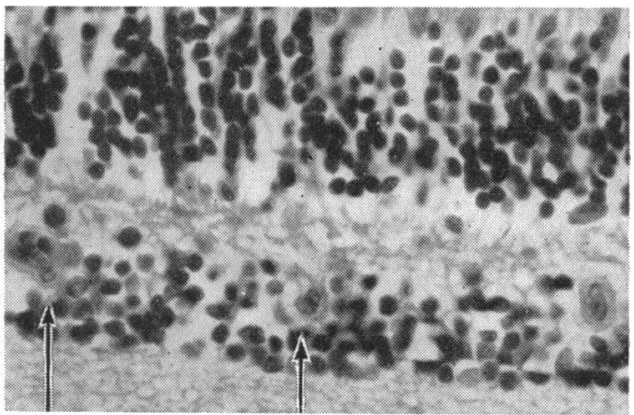

FIG. 27.-Case 2, section of retina showing capillary bed in fulminating hypertension. The capillaries are widely dilated and contain many red blood corpuscles; the endothelial cells are swollen. $\times 167$.

Hypertensive arteriolar necrosis occurs, and has a focal distribution, in fulminating hypertension. A necrotic arteriole is shown in Fig. 28, the lumen is empty and the greatly thickened wall contains a dissecting haemorrhage.

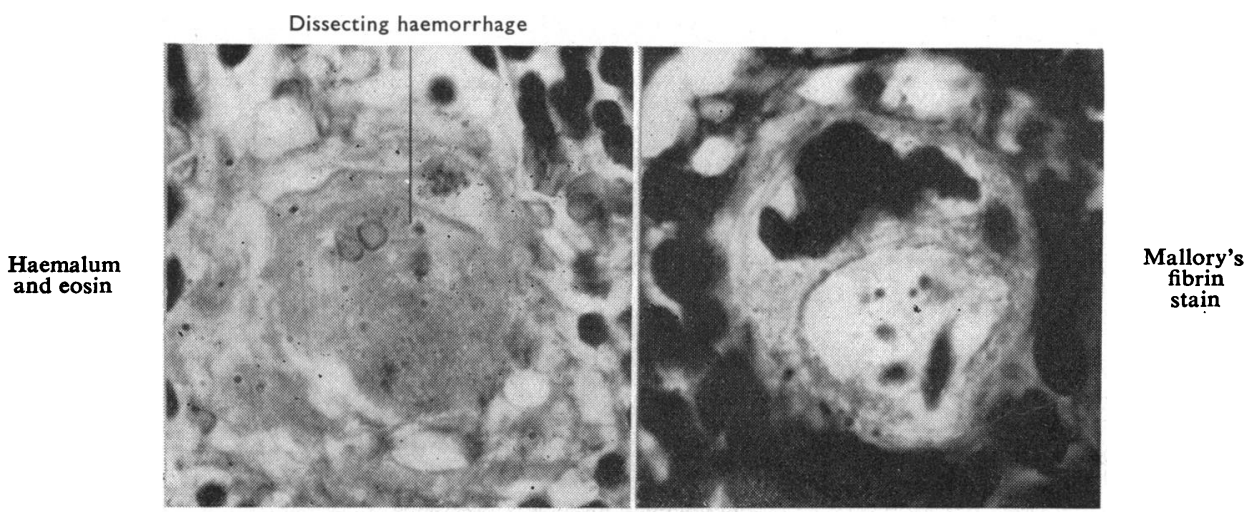

Fig. 28.-Case 2, adjacent sections from a necrotic arteriole in fulminating hypertension. The greatly thickened wall contains a dissecting haemorrhage. $\times 600$.

Fig. 29 (opposite) shows an arteriolar wall which is hyperplastic and swollen, part of the wall is excessively thick, and here two wall-cell nuclei show fragmentation, and a red thrombus lies against the necrotic wall. 


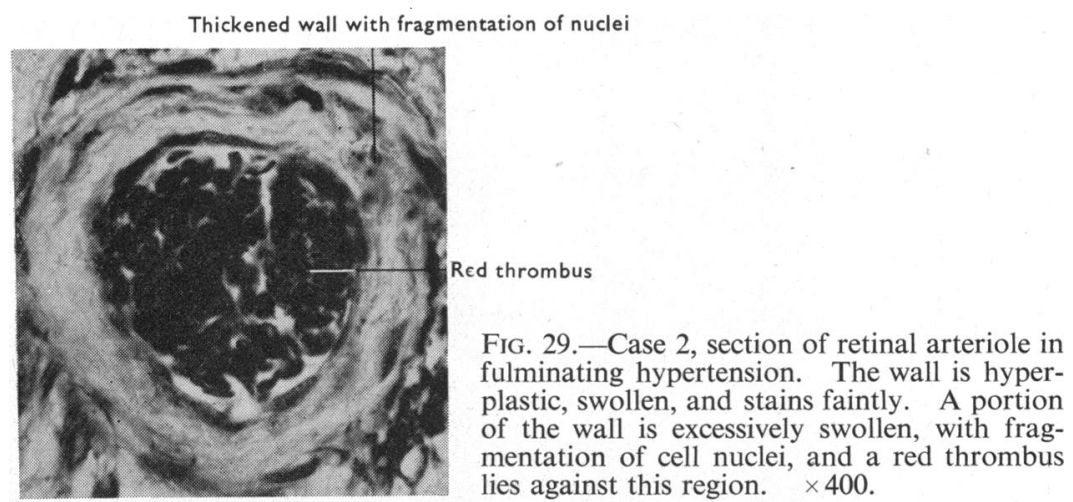

Thus, acute arteriolar necrosis is seen to occur in the vessels of the retina. Such lesions are known to be highly focal in distribution in other organs such as the kidney, and they are less likely to be numerous in the flat vascular system of the retina.

Fulminating hypertension is stated to occur in the younger age groups; it might be more accurate to say-in younger arteries, since it has been observed in a man of 70 years of age with a fundus picture of youthful type. Failing vision is one of the early symptoms, and the fundus picture is usually well established at the first examination. The diastolic blood pressure reaches very high levels $( \pm 160 \mathrm{~mm}$. $\mathrm{Hg})$. The ophthalmoscopic and histological features suggest that youthful arterioles, subjected to a very severe hypertensive stimulus, react strongly by hypertonus which is soon fortified by hyperplasia, as evidenced by the appearance of much new-formed elastic tissue; the tense cellular wall opposes mounting lateral blood pressure until overtaken by acute cell necrosis, arteriolar thrombosis, and vascular failure; this takes place before there has been time to develop defence by replacement fibrosis. Fortunately, cases of this severity are few in number.

Group 7. Severe Hypertension with Reactive Sclerosis.-The fundus picture in this group appears to represent a severe hypertensive stimulus in youthful vessels resulting in reactive changes; the reaction is progressing by way of hypertonus, and hyperplasia, into replacement fibrosis, and some degree of defence by sclerosis has been achieved.

Senile stigmata are absent from the fundus background (Fig. 30, overleaf). The disc is normal, or has a white haze which does not obscure the vessels on its surface. The arterioles are, in general, more tortuous than normal, and there is impediment to venous outflow at arterio-venous crossings. The veins are deeply congested, appearing full and markedly tortuous distal to the crossings. Small arterioles, and the more distal portions of the larger ones, are straight and narrow, indicating hypertonus. Some portions of the larger arterioles cause broad parallel zones of concealment as they cross the veins; and in thick-walled portions of the arterioles the colour of the blood 


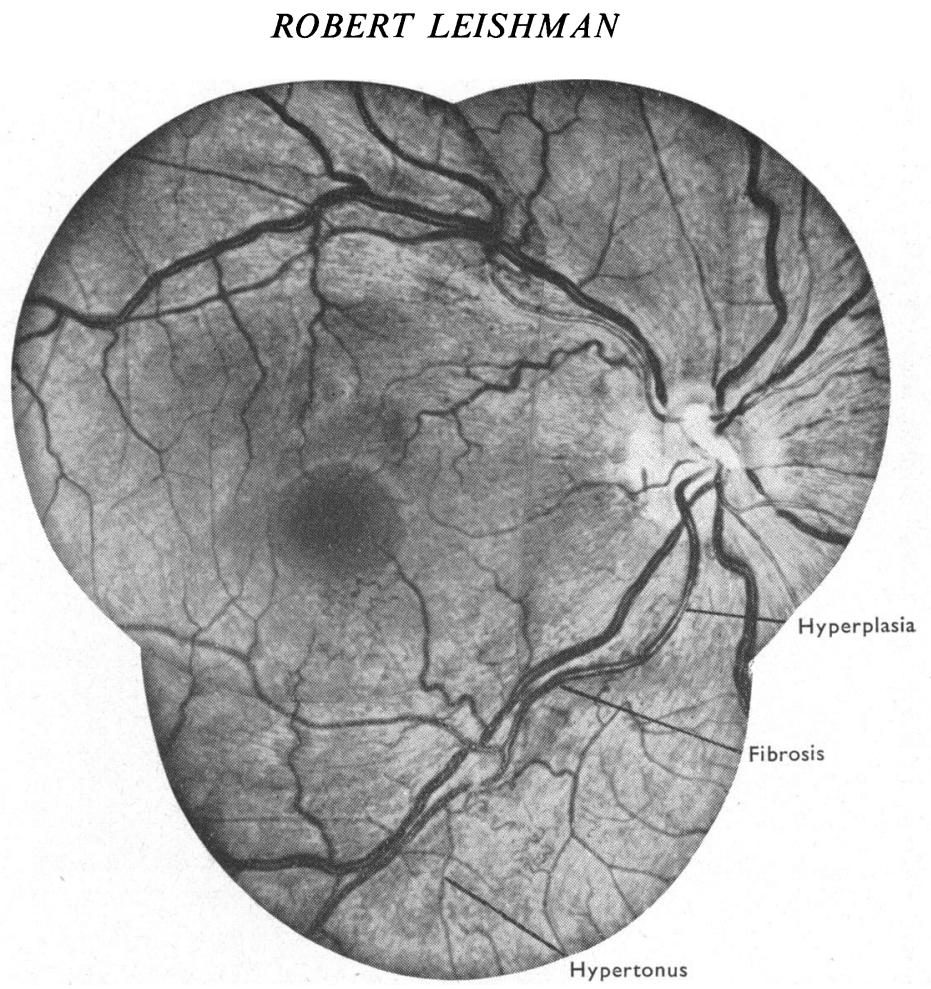

FIG. 30.-Fundus photograph in a case of severe hypertension with reactive sclerosis. Fibrosis, hyperplasia, and hypertonus are present in different portions of the same retinal arteriole.

column is excessively pale and its width finely variable but narrow, indicating hyperplasia. In other portions, the larger arterioles appear red and full, suggesting fibrosis.

In this type of case, by hypothesis, hypertension develops in vessels which are still of youthful rather than of aged type. The hypertensive disorder is not overwhelming but is persistent, and the diastolic pressure is maintained at a moderately high level since the peripheral vessels are reactive. Hypertonus is followed by hypertrophy and hyperplasia in some regions of the arteriolar wall, and in other regions these changes are superseded by replacement fibrosis. Ophthalmoscopically, the features of fibrosis, hyperplasia, and hypertonus, are observed concurrently in different parts of the same arteriole and its branches.

The following case illustrates the histological features of the condition, although the patient's age is well above the mean age of the group:

Case 3, male, aged 74 years. The left kidney was removed 25 years ago because of pyelonephritis. He was found to have hypertension 18 months ago, B.P. 270/130.

The right eye was excised on account of a malignant melanoma situated nasal to the disc. Fibrotic, hyperplastic, and hypertonic portions of the superior temporal artery were labelled on a fundus sketch before excision of the eye. The retina was photographed on the flat and later subjected to histological study. 
The fundus sketch (Fig. 31) made before excision of the eye is closely comparable with the photograph of the same artery in the cleared specimen of retina in bulk (Fig. 32). The same dilated and narrowed portions are identifiable in both illustrations. Parts of the arteriole which are dilated and presumably rigid in life remain wide in death; other parts where the wall is thickened by hyperplasia and the lumen is narrow and finely variable remain so in death; hypertonic portions which are straight and narrow in life are similar in death.

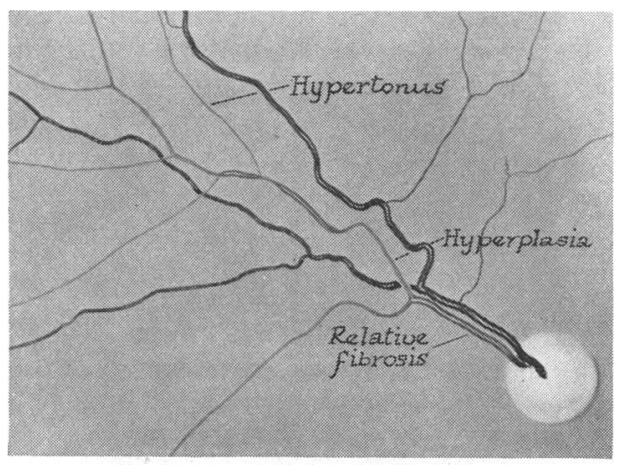

Fig. 31. Case 3, diagram of retinal arteriole in a case of severe hypertension with reactive sclerosis. Fibrotic, hyperplastic, and hypertonic portions were labelled before excision of the eye.

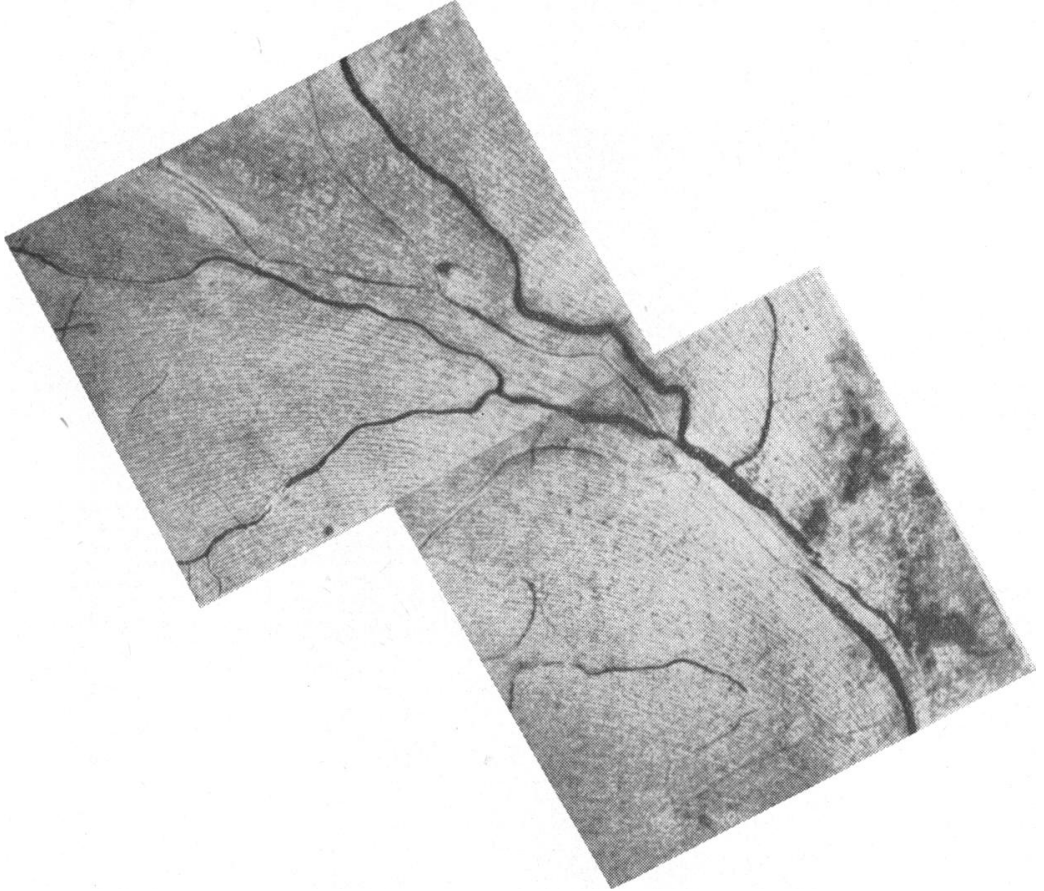

FIG. 32.-Case 3, flat preparation of retinal arteriole shown in Fig. 31, after excision of the eye. The dilated and narrowed portions are identifiable in both illustrations.

The histological sections of a segment of the artery labelled "relative fibrosis" in life had the following characteristics (Fig. 33, overleaf). The lumen was wide 
and the wall relatively thin. There was a relative diminution in the number of wall-cells. The wall was mainly collagenous, although elastic fibres were distributed throughout the collagen. The presence of multiple elastic fibres in the walls of a retinal arteriole at a point several millimetres from the disc is abnormal and suggests that the wall of the vessel has gone through a phase of reactive hyperplasia to reach the stage of relative fibrosis.

In segments labelled "hyperplasia" (Fig. 34), the lumen was more narrow and circular, and the wall thicker. The wall contained many new elastic fibres amongst the collagen; a considerable number of nuclei was present. All elements stained fairly well in contrast to the appearance in fulminating cases with hyperplasia of the supporting elements.

In segments labelled "hypertonic" in life, the lumen was fairly narrow and circular (Fig. 35). The wall was of normal thickness and cell content. A few elastic fibres were present even in relatively small vessels.

Apart from the early cases with generalized constriction of the retinal arterioles, and occasional cases showing fulminating features, the great majority of middle-aged people with hypertension fall into this group. Such patients cannot be classified as suffering from fulminating hypertension, yet the condition is more severe than the elderly fibrotic type. They usually give a history of 4 or more years' duration, and often the high blood pressure proves to be related to previous renal disease or hypertension of pregnancy. In hypertensive subjects, if given time, healthy vessels go through a process of adaptation from physiological hypertonus, by way of hypertrophy and hyperplasia, to partial replacement fibrosis. The appearance of these vascular changes of mixed type in the same retinal arteriole in hypertensive cases is consistent with moderately severe elevation of the diastolic pressure $( \pm 130 \mathrm{~mm} . \mathrm{Hg})$. This fundus picture is associated with a severe degree of hypertension, and usually occurs in young and middle-aged subjects whose vessels are reactive at the onset of hypertension. If, however, the stigmata of old age are present in the fundus of a relatively young person, the picture is that of involutionary sclerosis with hypertension, as already described.

A further extension of this picture may be conceived in which sclerosis has extended diffusely throughout the larger arterioles. In this way the patient might reach a state of acquired benign hypertension resulting from reactive sclerosis; the expected fundus picture would bear a close resemblance to, and might even be indistinguishable from, that of advanced involutionary sclerosis with benign hypertension, although differing from the latter in pathogenesis.

The hypothetical fundus pictures integrating hypertension and arteriosclerosis have been put to the test of clinical ophthalmoscopy, and it has been possible to segregate cases of these cardiovascular disorders into their respective groups by ophthalmoscopic examination alone in the great majority of cases. Some of the appearances are not capable of histological proof; but in others the test of histological examination has been applied when possible, and the resulting evidence, although insufficient to constitute 
(a)

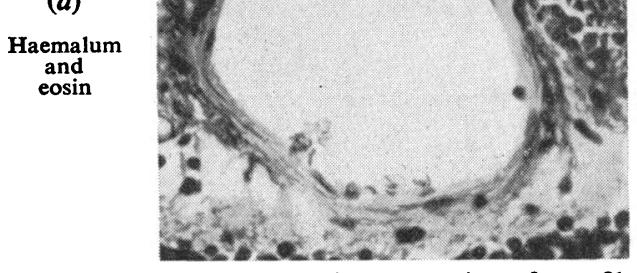

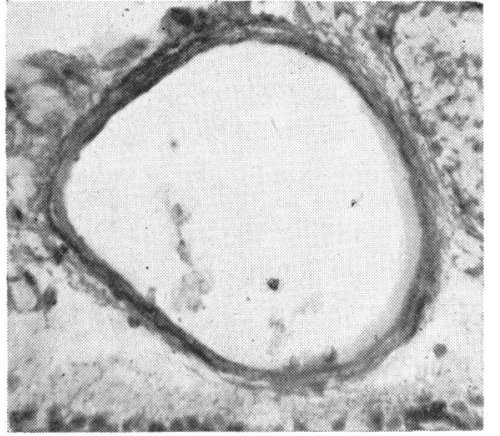

(b)

Weigert's

elastic

stain

FIG. 33.-Case 3, adjacent sections from fibrotic portion of retinal arteriole shown in Fig. 31.

The wall is thin and the lumen wide. The collagenous wall contains many elastic fibres. $\times 300$.

(a)

Haemalum and eosin

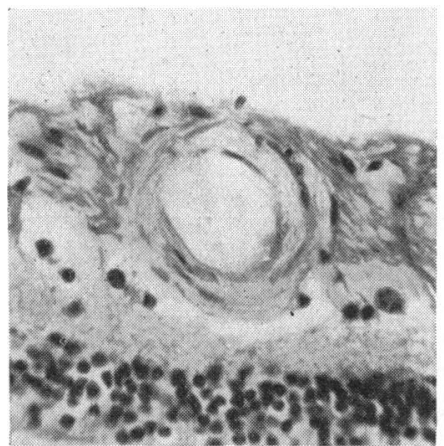

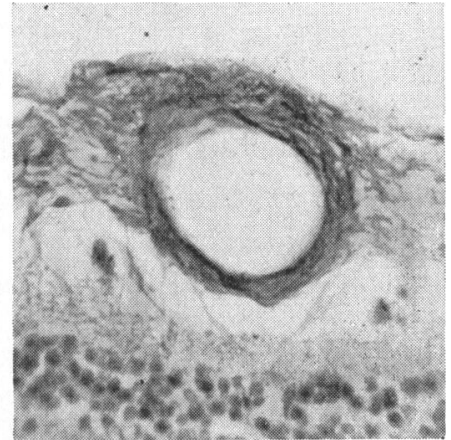

(b)

Weigert's

elastic

stain

FIG. 34.-Case 3, adjacent sections from hyperplastic portion of retinal arteriole shown in Fig. 31 . The wall is thick and the lumen relatively narrow. The thickened wall contains numerous elastic fibres. $\times 300$

(a)

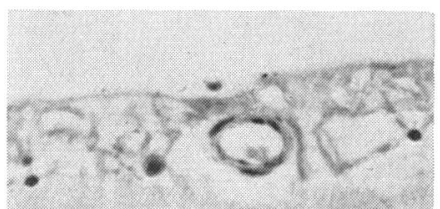

Haemalum and

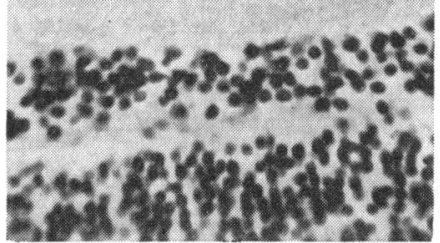

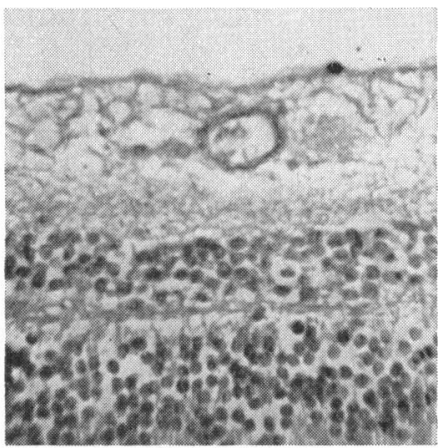

(b)

Weigert's

elastic

stain

Fig. 35.-Case 3, adjacent sections from hypertonic portion of retinal arteriole shown in Fig. 31 . The wall is of normal thickness and cell content, with a narrow lumen. Elastic fibres are present even in this small vessel. $\times 300$.

a proof, offers support to the hypothesis. The ophthalmoscopic interpretations derived from these studies and stated in basic terms of physiology and pathology, appear to have some meaning with regard to the assessment of the general medical condition of the patient. 
A further method of testing the hypothesis is to examine the frequency distribution of cases in the various groups determined by ophthalmoscopy in relation to general medical experience.

\section{Analysis of Clinical Data}

The population in these studies was drawn from cases of hypertension treated as in-patients in a general hospital and from other cases attending the ophthalmic department of the same hospital as out-patients. A sample of 122 consecutive cases of hypertensive disease was selected at random for statistical analysis. This sample included cases of senile involutionary sclerosis, with raised systolic but normal diastolic pressure. The fundus picture was the criterion of classification in all the cases, without reference to age or blood pressure readings.

The frequency distribution of the cases shows a fair measure of agreement with general medical experience (Table II). Benign hypertension (Groups 2 and 3-involutionary sclerosis with hypertension) accounts for the largest number. Next comes severe hypertension with reactive sclerosis (Group 7). These groups are regarded by physicians as a single homogeneous group (benign essential hypertension), in which the conditions have a common pathological basis and differ only in respect of the severity of the illness. This conception is not borne out in the present studies, since the groups present entirely different ophthalmoscopic features and the histological evidence suggests fundamental differences in pathogenesis. Fulminating hypertension is much less common; even so, the frequency shown in this series may be excessive, since cases of fulminating hypertension tend to become concentrated for treatment in hospital wards. There is a preponderance of females in all the groups of the sample.

TABLE II

ANALYSIS OF 122 CASES OF HYPERTENSION

\begin{tabular}{|c|c|c|c|c|c|c|c|c|}
\hline \multirow{3}{*}{$\begin{array}{l}\text { Ophthalmoscopic } \\
\text { Grade }\end{array}$} & \multirow{3}{*}{$\begin{array}{l}\text { Number } \\
\text { Cases of } \\
\text { in Grade }\end{array}$} & \multicolumn{2}{|c|}{ Sex } & \multicolumn{4}{|c|}{ Age (yrs) } & \multirow{3}{*}{$\begin{array}{c}\text { Mean } \\
\text { Age of } \\
\text { Cases in } \\
\text { Grade }\end{array}$} \\
\hline & & \multirow{2}{*}{ Male } & \multirow{2}{*}{ Female } & \multicolumn{2}{|c|}{ Male } & \multicolumn{2}{|c|}{ Female } & \\
\hline & & & & Range & Mean & Range & Mean & \\
\hline Involutionary Sclerosis & 14 & 5 & 9 & $46-76$ & 62 & $38-79$ & 65 & 64 \\
\hline $\begin{array}{lcc}\text { Involutionary } & \text { Sclerosis } & \text { with } \\
\text { Hypertension } & . & . .\end{array}$ & 62 & 25 & 37 & $39-83$ & 65 & $30-83$ & 64 & 64 \\
\hline Early Hypertension .. & 11 & 4 & 7 & $24-48$ & 36 & $27-62$ & 41 & 39 \\
\hline Fulminating Hypertension ... & 8 & 2 & 6 & $38-51$ & 45 & $26-58$ & 46 & 45 \\
\hline $\begin{array}{l}\text { Severe Hypertension with } \mathrm{Re}- \\
\text { active Sclerosis } \quad . .\end{array}$ & 27 & 11 & 16 & $50-69$ & 58 & $34-66$ & 50 & 53 \\
\hline Totals .. & 122 & 47 & 75 & & & & & \\
\hline
\end{tabular}


A perusal of the mean age is rewarding. The mean age of patients with involutionary sclerosis without hypertension is identical with that of patients with involutionary sclerosis with hypertension (64 years). Thus, although the characteristics of the retinal arterioles are in some respects opposite in the two groups, the cases seem to be drawn from the same age group of the population. This strongly supports the validity of the hypothetical fundus picture of hypertension in aged vessels. Furthermore, the mean age (64 years) shows that these cases of benign hypertension (Groups 2 and 3) are drawn from a different age group than the cases designated early hypertension in young vessels (mean age 39 years). It is commonly held amongst ophthalmologists and physicians that the most constant and characteristic sign of raised tension is a narrowing and straightening of the retinal arteries; this is certainly the case in young people, but not in elderly benign hypertensives, the group accounting for the majority of hypertensive cases. Again, the difference in mean age between the patients in Groups 2 and 3 (benign hypertension and involutionary sclerosis with hypertension: mean age 64 years), and Group 6 cases (severe hypertension with reactive sclerosis: mean age 53 years) supports the hypothesis that these groups are not homogeneous, but have different natural histories and different pathological bases. An interesting and unexplained feature of the cases in Group 7 (severe hypertension with reactive sclerosis) is that the mean age of the males is significantly higher than that of the females. In the males of this group hypertension may frequently be related to previous renal disease, whereas in a high proportion of females the hypertension has been noted first in a previous pregnancy. The maximum and minimum ages recorded in all the groups show that there is a wide range of individual variation with regard to the incidence of hypertension and arteriosclerosis.

While, therefore, these figures appear to be in close agreement with general medical experience, and give further support to the ophthalmoscopic method of grading such cases, they also reinforce the criticism that current medical classifications of hypertension and arteriosclerosis are inadequate.

\section{CONSECUTIVE OBSERVATIONS}

The ophthalmoscopic, histological, and statistical evidence so far adduced has in no respect revealed a need for reconsideration of the original working hypothesis; on the contrary, it has tended rather to confirm the reasoning. The next stage in the investigation is to assume the validity of the argument in order to test it further by determining whether or not it will help in elucidating other abnormal phenomena observed in the fundus.

\section{ATHEROMA}

The delineation of the composite fundus pictures resulting from hypertension and sclerosis has been completed without having had to consider the question of atheroma, although this is a very common type of arteriosclerosis known to occur sometimes, but not always, in association with hypertension. 
Atheroma has been defined as a patchy, degenerative lesion affecting arteries of all sizes, and characterized by swelling of sub-endothelial cells, with subsequent fatty deposition and associated fibrosis. The fibrosis is usually considered to be a secondary result of the lesion in the intima.

Fibrotic subjects often show a large atheromatous plaque in the central retinal artery in the optic nerve (Fig. 36) and this type of lesion presents some interesting features. There is always evidence of considerable replacement fibrosis throughout the outer coats of the arteries. In histological preparations, these outer coats retain a circular cross section which suggests rigidity. The endothelium is intact and encloses a circular but eccentric lumen. The lesion occupies the space between the endothelium and the rigid outer tunics. The distribution of the collagen in the outer coats suggests a primary replacement fibrosis rather than a reactive fibrosis secondary to the atheromatous lesion. At the same time, however, there is an excess of collagen in the subendothelial region occupied by the lesion. These appearances suggest that an intact and resilient endothelium has retracted from a previously rigid outer wall in order to contain a blood column of diminished volume, with resultant loosening of the sub-endothelial reticulum, swelling of sub-endothelial cells, deposition of fat, and formation of new collagen (Fig. 37).

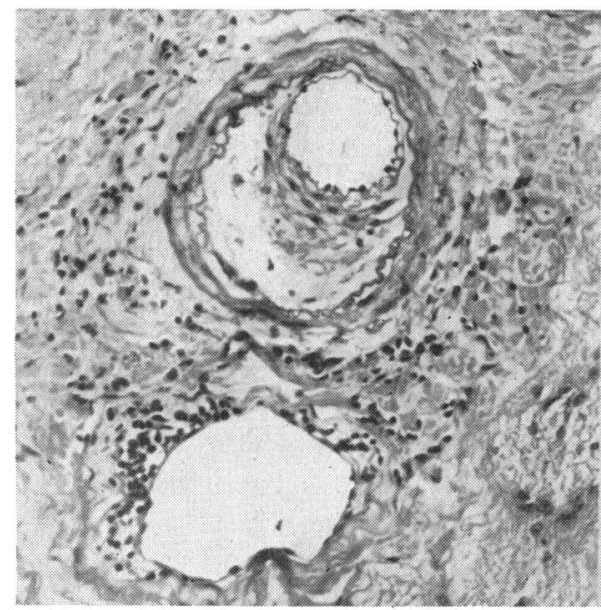

Fig. 36.-Section of central artery and vein in the optic nerve in a case of involutionary sclerosis with hypertension. The outer coats of the artery show a circular cross section. The endothelium is intact enclosing a circular but eccentric lumen. An atheromatous lesion occupies the space between the endothelium and the rigid outer tunics. The vein wall shows marked cellular hyperplasia. $\times 129$.

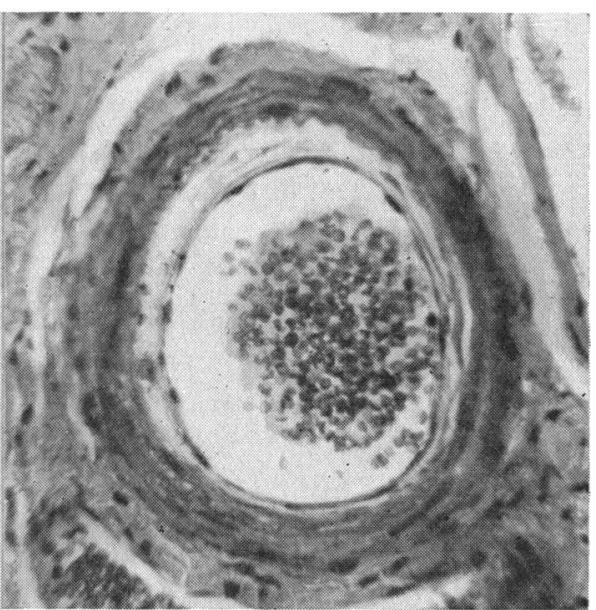

FIG. 37.-Section of central artery in the optic nerve in a case of involutionary sclerosis with hypertension: The outer tunics show replacement fibrosis and are circular in cross section. The intact endothelium is retracted from the rigid outer wall. Large subendothelial cells are present between the endothelium and the outer wall. $\times 172$.

In fulminating hypertension, the central artery in the optic nerve shows no evidence of involutionary sclerosis and remarkably little evidence of reactive change; it appears that the muscle coat is able to withstand extremely high diastolic pressures for a time without the appearance of significant com- 
pensatory changes in the vessel wall. In such a case, however, a lesion was observed which must be considered in this connexion. This lesion, which was found in a main retinal branch on the nerve head, is illustrated in Fig. 38. The outer part of the vessel wall describes a wide circle, although, since fibrosis is absent, it is unlikely to be rigid. The intact endothelium is retracted from a portion of the outer wall, and the lumen is narrowed and eccentric. The space between the endothelium and the main part of the wall is occupied by two enormously swollen cells. One is tempted to think that this is an acute type of atheromatous lesion resulting from a fortuitous and anatomically determined rigidity of the outer wall of the artery at the lamina cribrosa, and induced by a terminal fall of blood pressure in the vessel.

Developmentally, the essential part of an artery is the endothelial tube, and

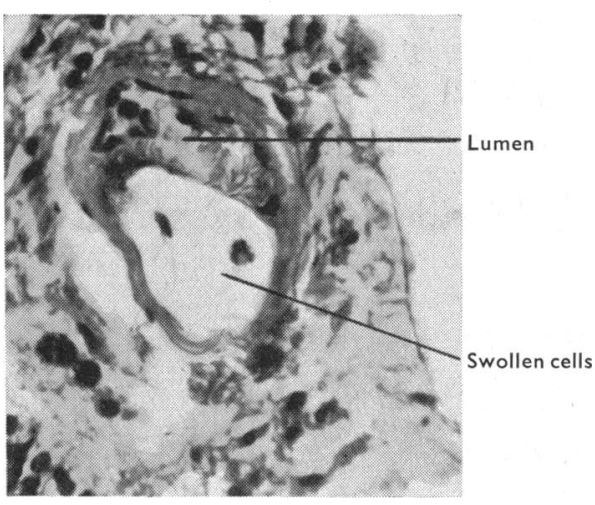

FIG. 38.-Section of retinal arteriole in a case of fulminating hypertension. The outer tunics are circular but not fibrotic. The intact endothelium is retracted from a portion of the outer wall. The lumen is narrowed and eccentric. The space between the endothelium and the outer wall is occupied by two swollen cells. $\times 400$.

its function is to contain the blood column; the outer coats of the wall are added later. The endothelial tube can be conceived to retain its resilience when the outer wall has become rigid from fibrosis, and, if the pressure and volume of the contained blood were reduced for a significant time, the endothelium might attempt to contain the diminished blood column by partial retraction from the rigid outer wall of the vessel. This would result in cloudy swelling of sub-endothelial cells, reversible at first with return of increased blood pressure and volume, but later irreversible after deposition of fat and formation of new collagen fibres around the retracted endothelium. If this reasoning is correct, fibrotic rigidity of the outer wall of the vessel becomes the predisposing factor, and relative diminution of blood pressure and volume an exciting factor, in the production of the atheromatous lesion. Such a lesion, once established in a small vessel, will tend to shut off more distal portions of the vessel from the full systemic pressure and will allow a further "seeding" of the atheromatous process in other fibrous portions in a distal direction.

In the course of the ophthalmoscopic studies already described, it was frequently observed that fibrotic elderly vessels are prone to lesions causing partial occlusion. In some cases the lesion is small; it is usually of sudden onset, and may be of transient duration, lasting only a few hours or days. The occlusion is usually partial, and, distal to the lesion, the arteriole runs a straighter course and the colour of the blood column soon approximates to that of venous blood, suggesting lower pressure and deficient oxygenation. 
Such an obstruction has been observed to resolve, the arteriole regaining the width, colour, and tortuosity of a fibrotic hypertensive vessel; and, after some weeks, a permanent small atheromatous patch appears at the site of the original lesion. This type of lesion, since it occurs in fibrotic portions of a vessel, cannot be attributed to spasm; nor, with resolution of the initial lesion, has an embolus been found lodged in more distal branches.

At the site of such a permanent atheromatous patch in a retinal arteriole, an opaque white mass is seen to erode or even obscure a portion of the blood column; in cases with a more extensive lesion the appearance may be that of pipe-stem sheathing. The mass is bounded laterally by straight parallel edges which are continuous with the outer limits of the blood column at either end of the lesion, so that it appears to be plugging the vessel. This has led to the description of the condition as an "embolism", a misleading conception in most cases. True embolism does occur, but usually in young subjects with bacterial endocarditis. Most cases of so-called embolism of the retinal arteries occur in elderly sclerotic patients who have no cardiovascular lesions likely to produce emboli; the appearance is much more often the herald of peripheral circulatory failure.

If these lesions are due to failure of peripheral pressure and volume in rigid vessels, then therapy should aim at promoting gentle cardiovascular stimulation, and the use of vasodilators would be contraindicated.

The effects of the persistent partial atheromatous occlusion of a fairly large retinal arteriole may be seen in Case 4 (Fig. $39 a$ and $b$ ), which was

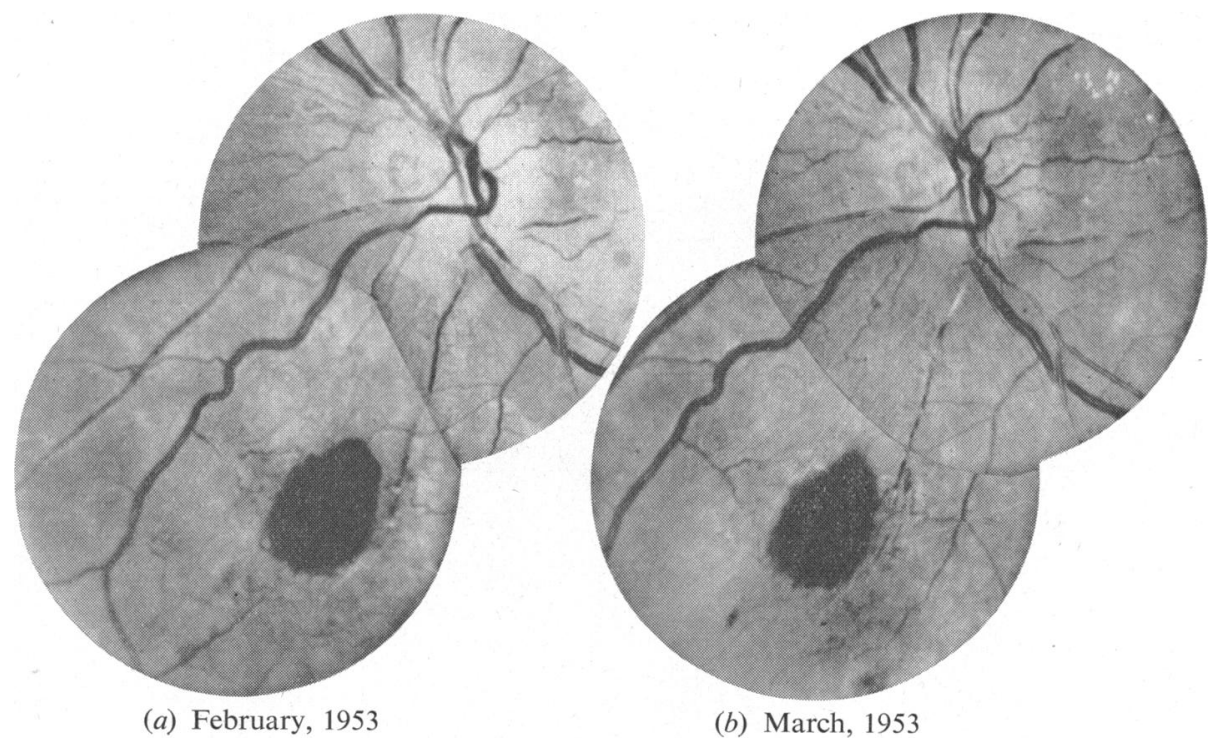

Fig. $39(a$ and $b)$.-Case 4 , fundus photographs in treated fulminating hypertension, taken at an interval of 6 weeks. A large haemorrhage has resulted from partial atheromatous occlusion of a retinal arteriole. Seeding of the atheroma in the rigid vessel with low pressure can be seen distal to the initial lesion. 
photographed repeatedly during a period of 8 months to observe the progress of the lesion.

Case 4, female, aged 50, fulminating hypertension when first seen (B.P. 260/170). Partially controlled by hexamethonium therapy. Segments of the retinal arterioles became fibrotic in appearance over the course of 2 years. An attack of cerebral thrombosis occurred 18 months after onset of symptoms and hypertension.

A large haemorrhagic mass appeared in the lower nasal quadrant of the retina; a main retinal vein lay on each side of the mass. A vessel containing venous blood ran from the mass towards the disc and, on tracing this vessel centrally, short white threads were noted to arise from it at intervals. These were recognized as the visible remnants of thrombotic arterioles, and the main vessel now containing venous blood must therefore have been an arteriole. This vessel was traced centrally to a white atheromatous patch near its point of origin from the inferior temporal artery. The veins on either side of the haemorrhagic mass were distended and their venules lying towards the mass were also distended and varicose.

The interpretation of this lesion appears to be that an atheromatous patch had formed near the origin of a fibrotic arteriole, causing a severe degree of partial occlusion of the vessel. The blood flow was sufficiently maintained to prevent thrombosis, but the pressure in the fibrous and partially occluded vessel was low. This resulted in a "stuffing-in" of blood from the veins on both sides of the area of arterial supply, even to the extent of varicosity and rupture of terminal venules and capillaries, an appearance which may be interpreted as a red infarct. It is of interest that further atheromatous lesions appeared later in the distal portion of the arteriole in which the tension was low.

By analogy, partial occlusion of the central retinal artery within the optic nerve could produce a picture of incipient venous thrombosis, a picture which is probably due to atheroma, and which may also be interpreted as a red infarct of the retina as in Case 5 (Fig. $40 a$ and $b$, overleaf):

Case 5, male, aged 40 (B.P. 160/95). The fundus picture in the right eye is that of involutionary sclerosis; hypertension is minimal. At first sight, the left eye shows a picture of incipient venous thrombosis, but defective filling of the retinal arteries is a striking feature.

It is conceivable that such a picture could occur in fibrotic vessels, whether the fibrosis was of involutionary or reactive type. The advent of papilloedema and retinal haemorrhages in a case of severe hypertension with reactive sclerosis, cotton-wool patches in the fundus, and moderately high diastolic pressure, could easily be taken to herald the onset of the "malignant phase" of hypertension, if fibrotic changes in the retinal arterioles were not taken into account.

This fundus picture of incipient venous thrombosis is not uncommon in elderly benign hypertensive cases in whom the heart is failing; it has also been observed in sclerotic subjects, no longer hypertensive, but known to have suffered previously from high blood pressure.

$43+$ 
Fig. 40 ( $a$ and $b$ ).-Case 5, fundus photographs of both eyes in the same patient. The right eye shows involutionary sclerosis. The left eye shows partial occlusion of the central retinal artery with oedema of the disc and venous congestion with haemorrhages.

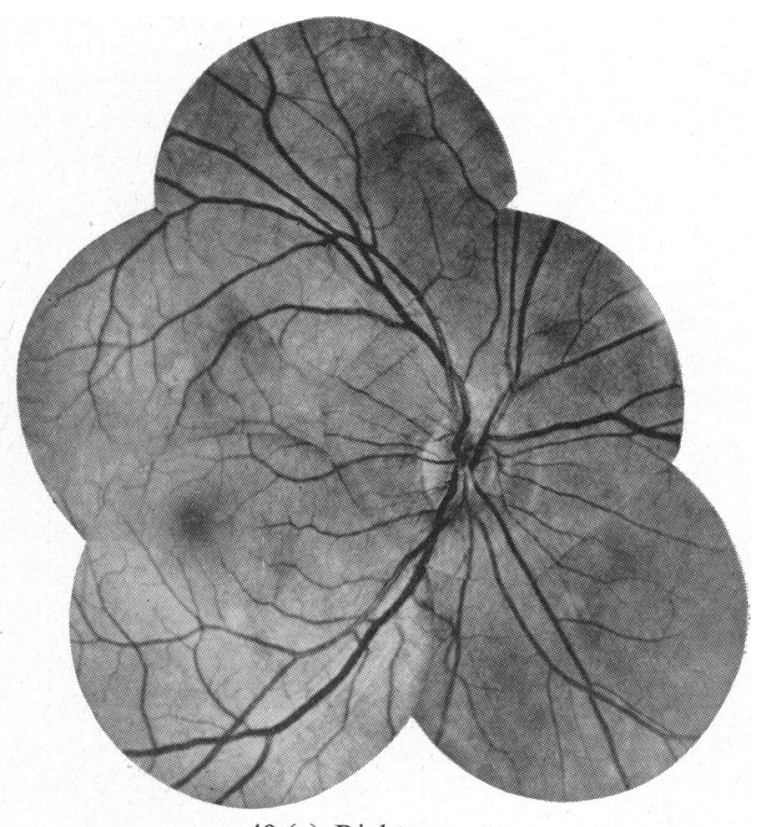

40 (a) Right eye

Not infrequently, in elderly benign hypertensive patients, the fundus picture in one eye is that of involutionary sclerosis with hypertension; the arteries show fibrotic dilatation without retinopathy, and the systemic blood pressure is of the order of $200 / 120 \mathrm{~mm}$. $\mathrm{Hg}$. The second eye shows a degree of optic atrophy; the retinal arteries are relatively pale, straight, and narrow, with disseminated patches of atheroma, and there may also be numerous small retinal haemorrhages and hard white exudates. Some of these features are illustrated by Case 6 (Fig. $41 a$ and $b$, overleaf):

Case 6, male, aged 67, benign hypertension for 6 months, treated with phenobarbitone. While on holiday, he received a course of hypotensive therapy on account of headache and visual disturbance. Vision deteriorated rapidly, and when he was seen in hospital 4 days later, a fundus picture of central artery occlusion was present in the right eye, and there was no perception of light. The picture in the left eye was that of involutionary sclerosis with minimal hypertension; 2 months later an atheromatous patch had developed in the inferior temporal artery of the right eye.

Hypotensive therapy appeared to precipitate the loss of vision in this case. This was discontinued, and replaced by a regime of moderate activity and controlled anticoagulant therapy. There has been slight recovery of vision in the right eye and no venous complications have arisen during 6 months. The fundus pictures in the two eyes may be compared in photographs taken 6 months after 


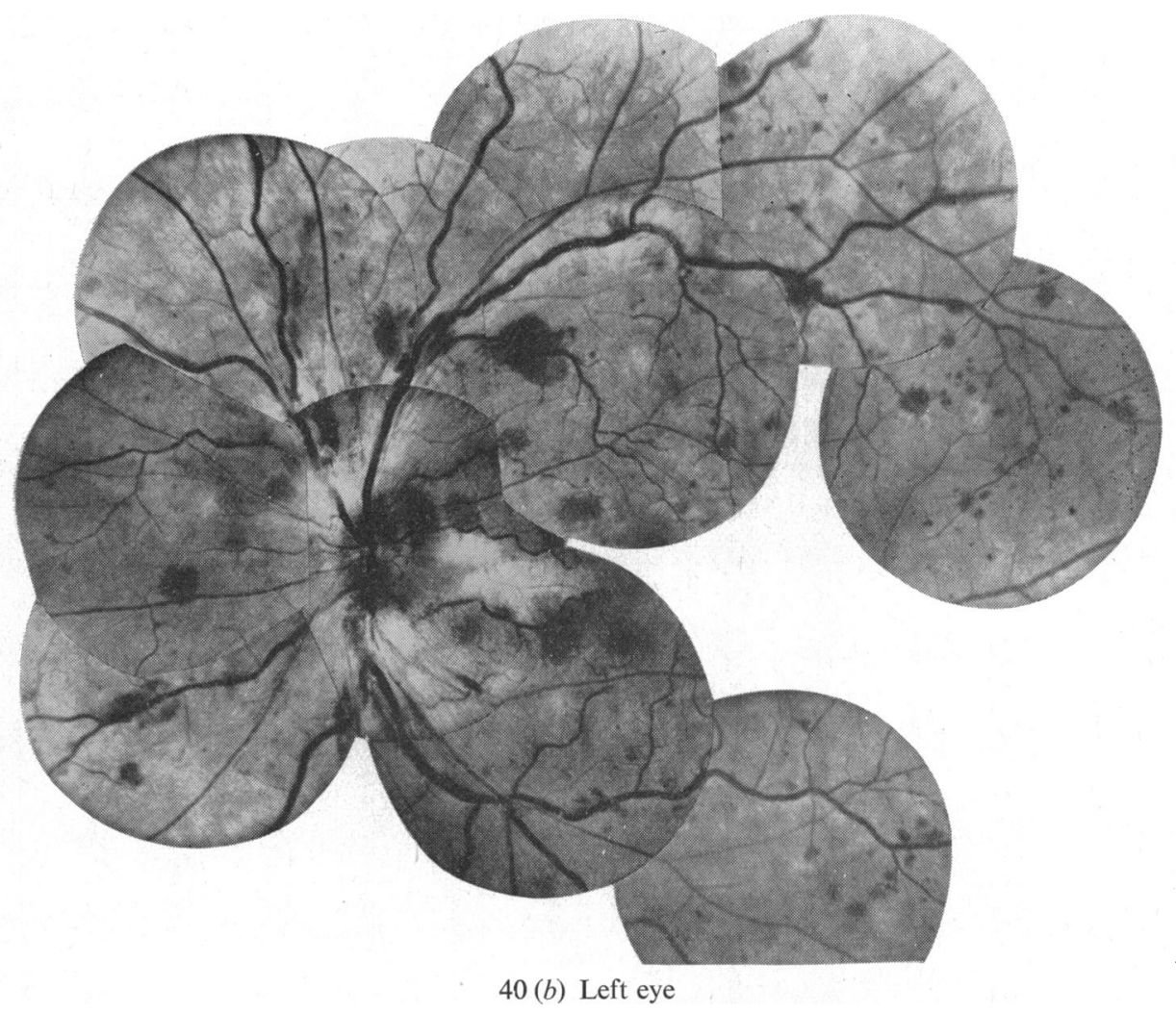

the occlusive incident (Fig. $41 a$ and $b$, overleaf). The appearance of the right eye is consistent with partial atheromatous occlusion of the central retinal artery in the optic nerve. The systemic blood pressure appears to be shut off so that there is a diminished pressure and volume of blood in the retinal vessels of the affected eye; atheromatous lesions have subsequently developed distal to the initial lesion in arteries known to be fibrous and now containing low pressure.

\section{VenOUS ABNORMALITIES}

Central Retinal Vein.-A striking feature associated with atheroma of the central artery in the optic nerve is a marked cellular hyperplasia which occurs in the walls of the accompanying veins (Fig. 36). The vein wall adjacent to the arterial lesion is thickened and contains numerous cells of endothelial appearance which, however, do not show mitosis (Fig.42, overleaf).

On casual inspection the cells are readily taken for lymphocytes, but on closer examination they are found to resemble endothelial cells. Clumps of these cells lie in the tissues of the nerve between the veins and the arterial lesion (Fig. 43, overleaf), as if they were migrating from the vein towards the lesion. Similar cells can also be identified within the lesion in the artery wall, and, here and there, one attains enormous size and appears as a foamy phagocytic cell (Fig. 44, overleaf). It seems as if the atheromatous lesion 


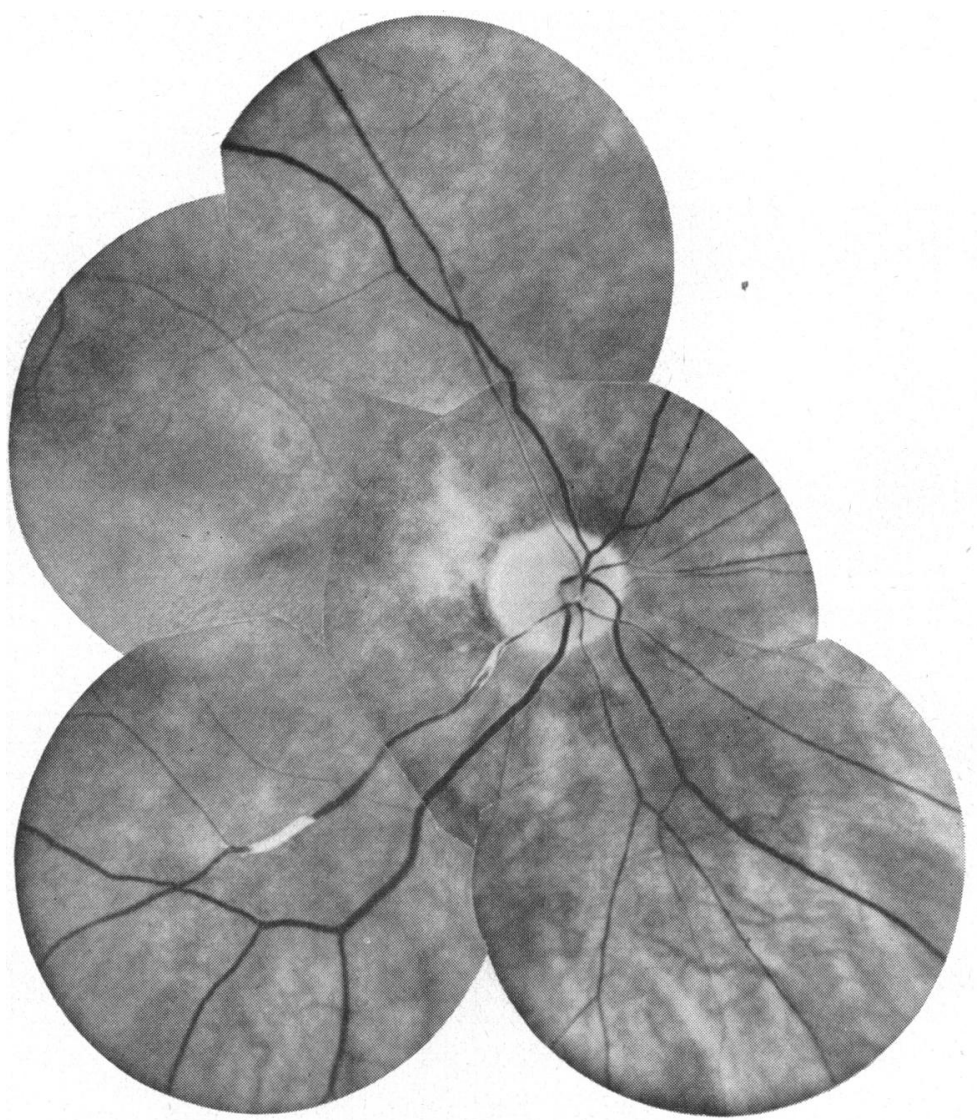

41 (a) Right eye

FIG. $41(a$ and $b$ ).-Case 6, fundus photographs of both eyes in a case of involutionary sclerosis with hypertension. In the right eye, occlusion of the central retinal artery was followed by the development of atheromatous lesions in a fibrous retinal arteriole.

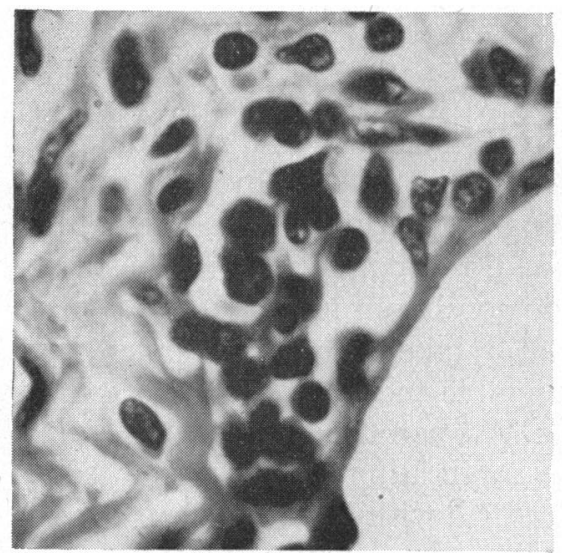

FIG. 42.-Section showing detail of endothelial proliferation in wall of vein shown in Fig. 36. $\times 600$.

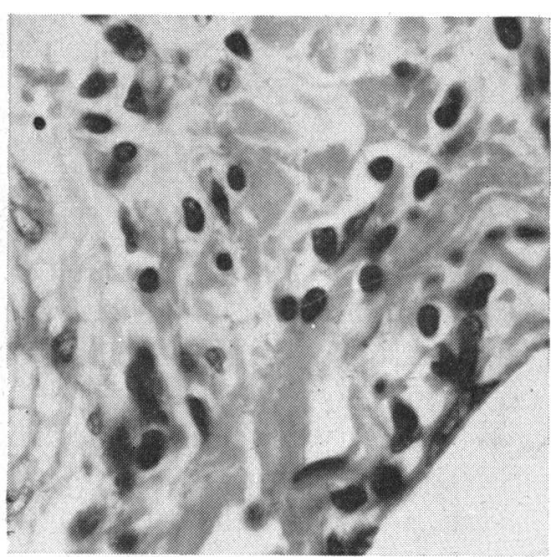

FIG. 43.-Section showing detail of migration of venous wall-cells shown in Fig. 36. $\times 600$. 


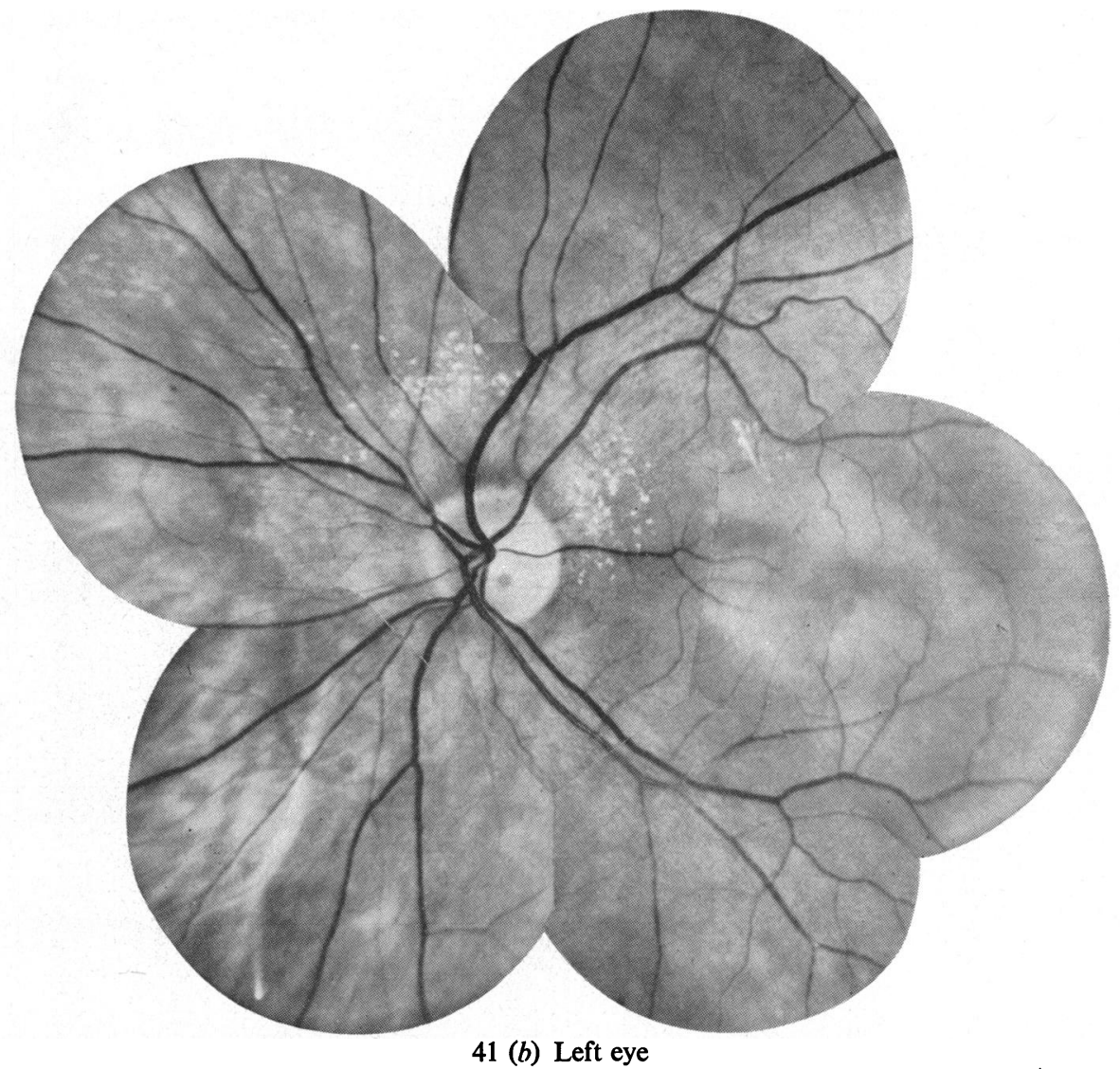

(a)
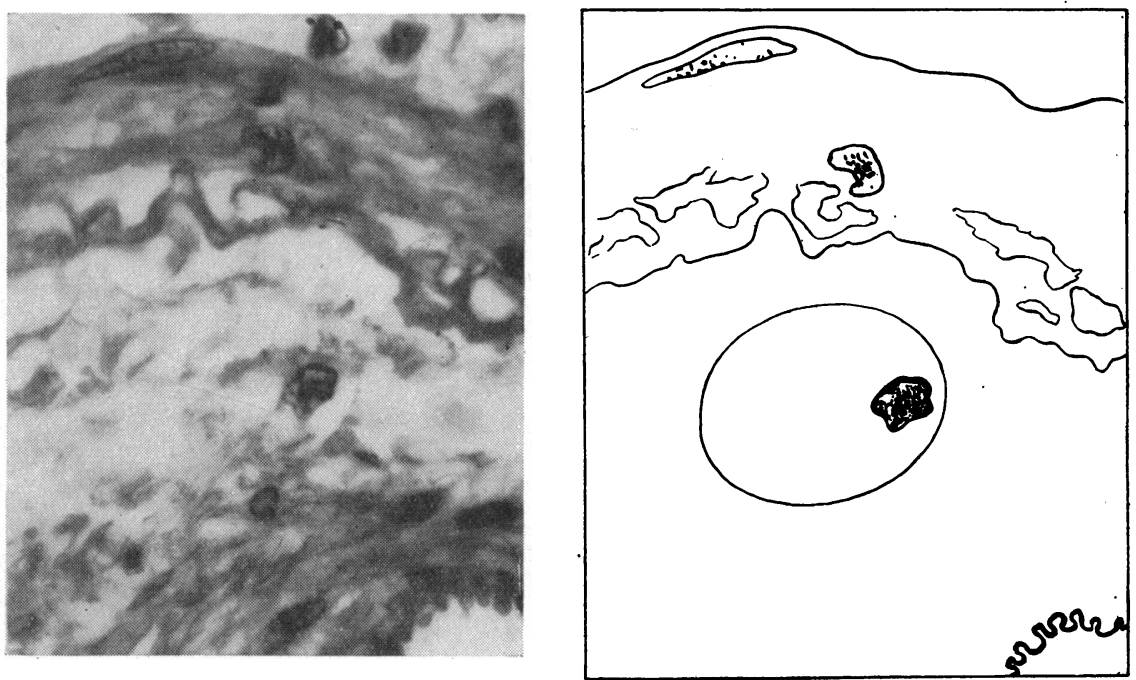

(b)

FIG. $44(a$ and $b$ ).-Section and key diagram showing foamy phagocytic cell in the atheromatous lesion seen in Fig. $36 . \quad \times 600$. 
calls forth a vigorous response on the part of the endothelial-like venous wall cells, which increase in number and appear to migrate towards the artery in an attempt to buttress and scavenge the degenerate region of the wall.

It has been suggested that narrowing of the lumen of the central artery in the optic nerve by atheroma occurs as a result of retraction of the endothelium from the rigid outer wall of the vessel. It is conceivable that increments of such retraction over a period of time would result in gross narrowing of the arterial lumen. In consequence, the cellular hyperplasia which occurs in the walls of adjacent veins would be greatly stimulated and, since outward expansion would be restricted by the surrounding nerve tissue, the prolific increase in cells would tend to reduce the lumen of the vein with corresponding obstruction to venous outflow (Fig. 45).

FIG. 45.-Section of central retinal artery and vein in the optic nerve, showing an advanced atheromatous lesion in the artery and consequent cellular hyperplasia in the vein wall with marked reduction of the lumen. $\times 100$.

The foregoing investigations into the behaviour of the central retinal artery and vein within the optic nerve emphasize the close relationship between arterial ischaemic and venous congestive disturbances arising in that situation, and the inferences drawn from them indicate a line of reasoning which appears to elucidate the pathogenesis in cases such as the following.

Three cases have been observed in which incipient venous thrombosis followed cataract extraction. In each case, the lens had been removed intracapsularly with peripheral iridectomy. Convalescence was apparently normal, and, about 4 weeks after operation, the visual acuity with correcting lenses reached $6 / 9$ and N5. Over a period of 2 to 4 weeks this gradually deteriorated to 6/60. Papilloedema and signs of incipient venous thrombosis appeared in the fundus. There was no apparent explanation for the thrombosis.

When this occurrence is re-examined in the light of the foregoing investigations, a probable interpretation of the pathogenesis can be offered. The ocular section and enforced inactivity could be conceived temporarily to diminish the peripheral pressure in the retinal arteries, precipitating transient atheromatous occlusion in the central artery and embarrassment in the venous circulation. With recovery of intra-ocular pressure and an ambulant 
regime, adequate circulatory pressure would be restored, although a permanent atheromatous lesion had become established in the central artery. This would initiate a hyperplastic response in the central vein, and would lead after a latent period, to mechanical narrowing of the lumen and to the development of a fundus picture of incipient thrombosis.

If this reasoning is valid, treatment should be directed towards maintaining peripheral pressure by a regime of moderate physical activity and towards preventing venous thrombosis by controlled anticoagulant therapy. Following this line of treatment, the visual acuity recovered steadily in all three cases to 6/9 and N5, and has been maintained at this level in the first case for 18 months. Although vision is still good, in two cases pallor has developed in the optic disc and the retinal arteries are attenuated.

Retinal Veins.-In hypertensive and sclerotic cases abnormal features are also observed in the retinal veins. A large vein passing close to a mass of hard-edged white exudates, may show a fusiform dilatation. The portion nearest to the mass of exudates may show a region of dense white sheathing in its wall. Unlike an atheromatous patch which appears to erode the blood column of a rigid arteriole, the white patch in the vein seems to lie in a thickened wall encrusting the blood column. The white material lies irregularly in the vein wall, and in disposition bears a close resemblance to the nodes of pigment which appear round the veins in retinitis pigmentosa. Such a lesion was observed during a period of more than 12 months in a case of severe hypertension with reactive sclerosis, and photographs were taken at frequent intervals (Fig. 46, overleaf). The hard white exudates were seen gradually to clear from the retina, and at the same time the sheathing around the vein became more dense. After the retina was free from exudates, the white node in the vein wall was observed to diminish in size, to decrease in density, and ultimately to disappear.

In histological sections taken from cases of severe and fulminating hypertension, the venous picture is often more striking than the arterial picture. The veins usually show deep congestion, and in places there may be a great increase in the number of wall cells (Fig. 47, overleaf). There is no evidence of mitosis in such a region, and on cursory examination these accumulations of cells in the wall might be dismissed as "lymphocytic infiltrations". On detailed examination the cells appear to be endothelial in type. Time and again, the impression has been gained that such cells are multiplying without mitosis in situ in the vein wall, and this appearance has come to be regarded hypothetically as hyperplasia of the wall cells. Sometimes in the lumen of a vein, and adhering to the hyperplastic portion of the wall, a large mass of fused platelets indicating a pale thrombus is observed (Fig. 48, overleaf). The hyperplastic reaction may or may not involve the whole circumference of the vein, and usually extends for a limited distance along its length, the wall thereafter becoming normal again in appearance and cell content. The histological picture suggests a node of cellular hyperplasia. 


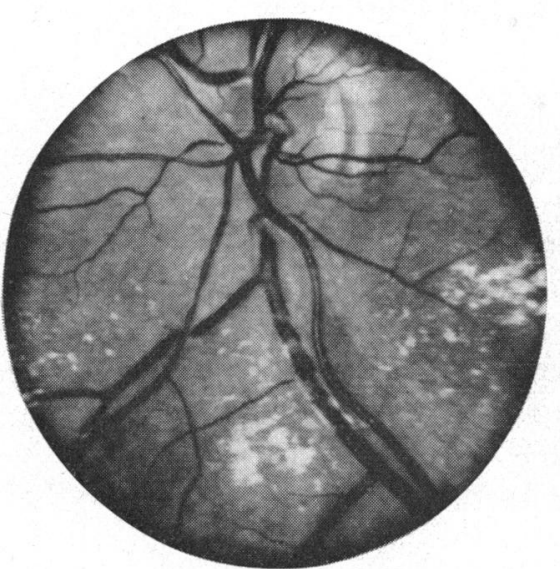

(a) January, 1953

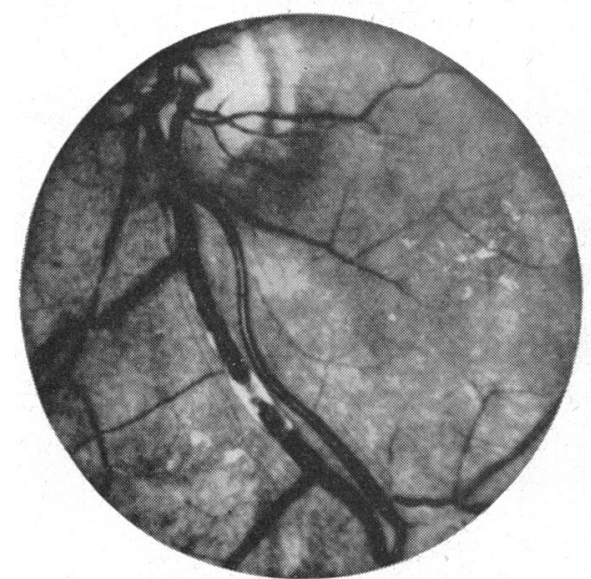

(c) June, 1953

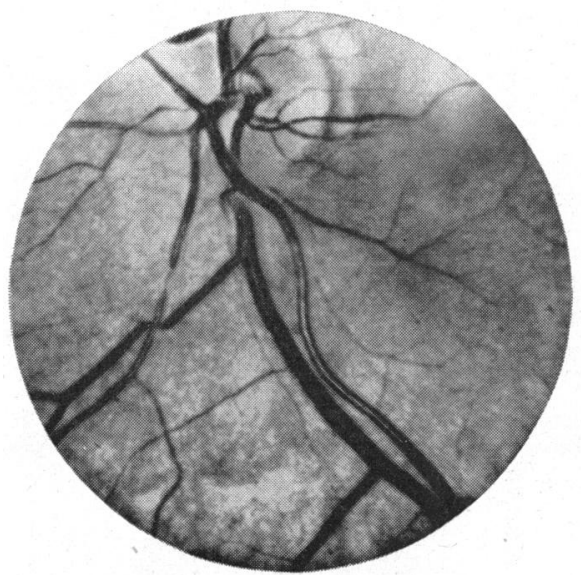

(e) October, 1954

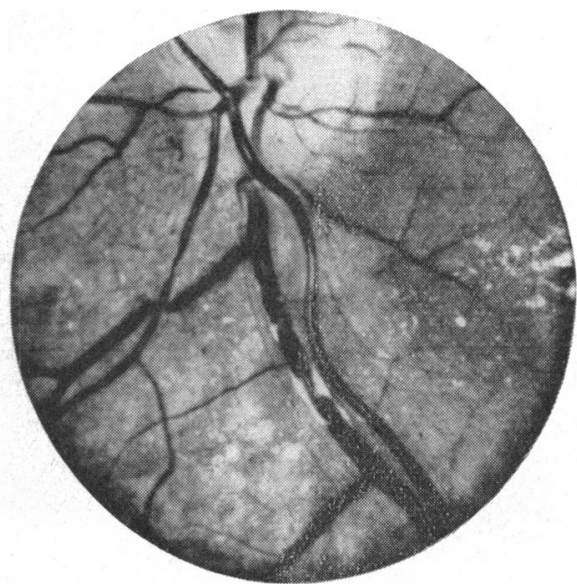

(b) April, 1953

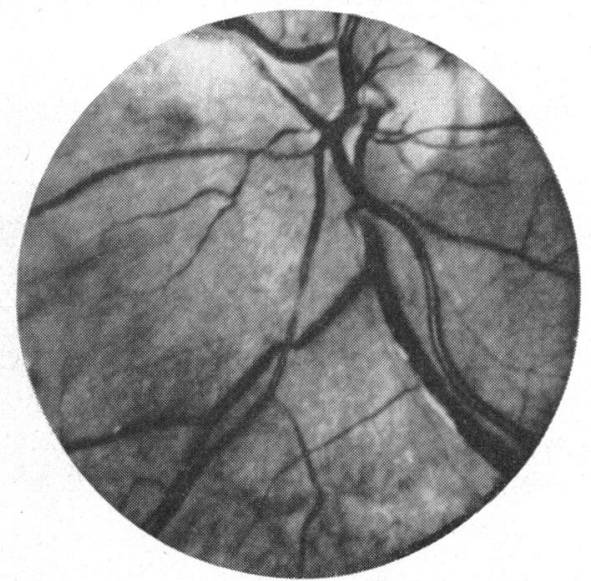

(d) December, 1953

FIG. 46.- Serial fundus photographs at intervals of 2 to 9 months in a case of severe hypertension with reactive sclerosis. A patch of hard white exudate lies near a large vein which shows white sheathing. As the exudate clears from the retina, the sheathing around the vein becomes more dense. After the retina is free from exudate the white node in the vein wall diminishes in size and ultimately disappears. 


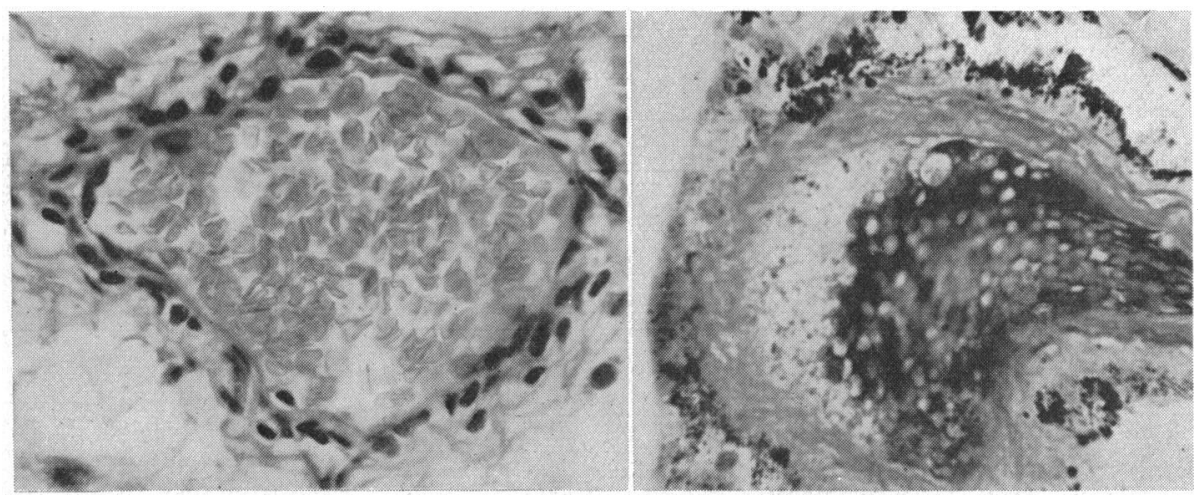

Fig. 47.-Section of retinal vein in a case of severe hypertension with reactive sclerosis. The vein shows marked hyperplasia of the cells of the wall. $\times 400$.

Fig. 48.-Section of retinal vein in same condition as Fig. 47. A mass of fused platelets forms a pale thrombus lying against a hyperplastic portion of the vein wall. $\times 400$.

Serial sections have revealed interesting features in such nodes of hyperplasia. Throughout a short length of the wall, the wall-cells are excessive in number and are separated to contain a wide irregularly-branching intramural channel. This channel is lined by wall cells which are large and rounded in appearance. The cytoplasm of these cells lining the channel contains granules of pigment (Fig. 49, overleaf). Investigations showed that the pigment was not haemosiderin, but was probably melanin.

It is suggested that in life this intra-mural channel had contained material phagocyted from deep retinal effusions, and that this feature in the vein wall represents a node for disposal of the transported material. The cells lining the channel are large and rounded, suggesting that the active phase was still in progress at the time of death, but the material which had occupied the channel must have been removed in processing the section. The node in the vein wall is comparable in extent to the regions of white sheathing already described to occur in the wall of a large vein near a patch of hard white exudates in severe cases of hypertension.

\section{RETINOPATHY}

In the preceding pages it has been possible to recognize and classify the fundus pictures due to hypertension and sclerosis in the retinal vessels without taking much account of the degenerative changes which occur within the retina itself in some of these cases. Sooner or later, however, haemorrhages and exudates appear in addition to the features of vascular hypertension and sclerosis, and the term retinopathy has been given to such a fundus picture.

Clinically, exudates are either of the small, round, hard, white variety, lying deep in the retina, or of the large, soft-edged, white type, described as "cotton-wool" patches, which often lie superficially in the retina obscuring the vessels. Haemorrhages and hard and soft exudates tend to appear early in fulminating or severe cases of hypertension in youthful vessels, and their appearance is usually associated with levels of diastolic pressure greater than $130 \mathrm{~mm}$. Hg. In fibrotic cases, these features usually appear at a late 

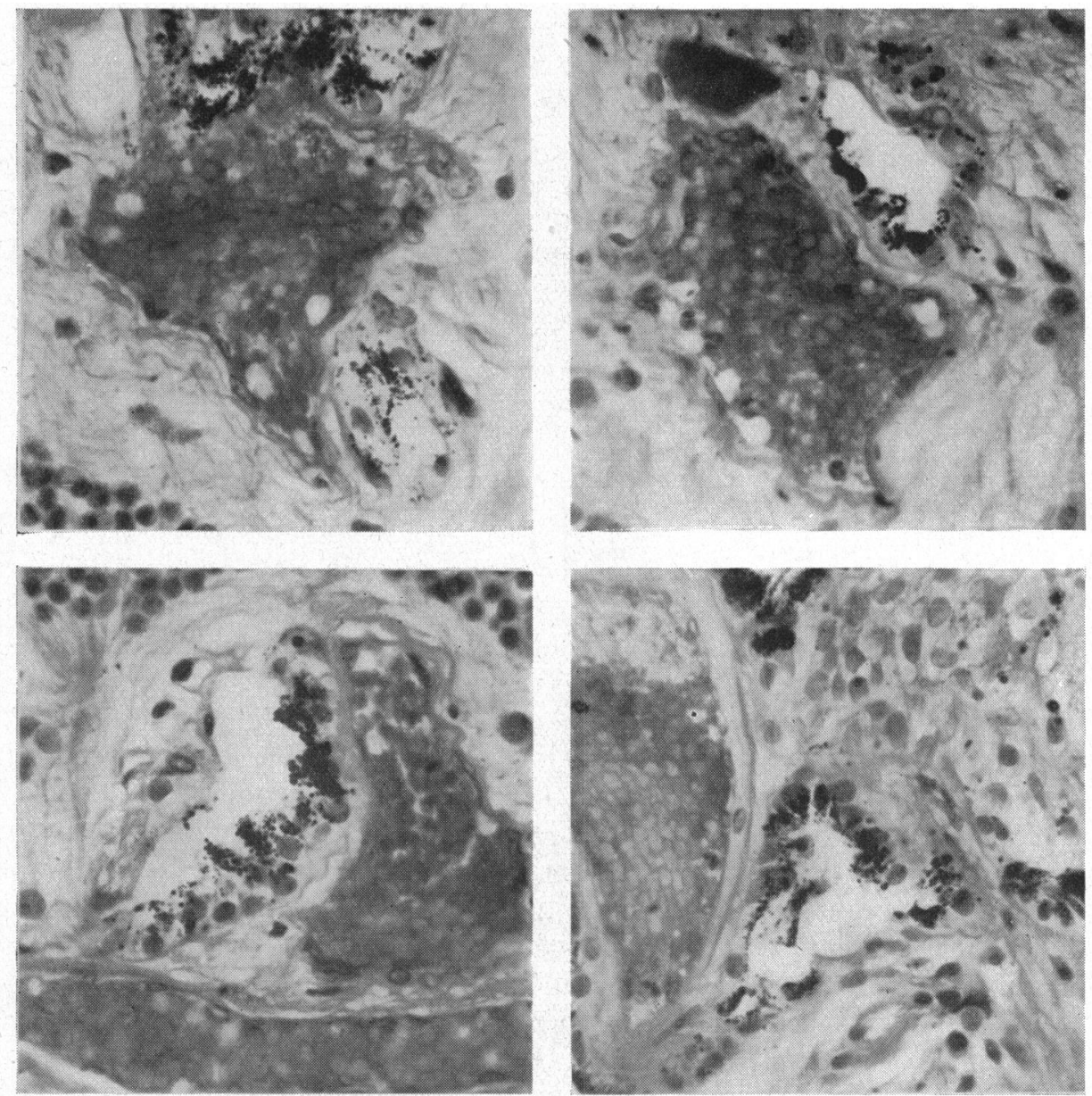

FIG. 49.- Serial sections of retinal vein in a case of severe hypertension with reactive sclerosis. The vein wall shows hyperplasia. The wall cells are separated to contain a wide irregularlybranching intra-mural channel. The cells lining the channel are large and rounded, and their cytoplasm contains melanin pigment. $\times 530$.

stage of the illness, and their appearance is to be related to relative failure of peripheral pressure and volume in rigid vessels; the relative absence of retinopathic changes in fibrotic cases with diastolic pressures of $\pm 140 \mathrm{~mm}$. $\mathrm{Hg}$ is significant.

The cause and meaning of these retinopathic changes are, however, still far from clear, since the correlation of the ophthalmoscopic appearances with the histological features has proved most difficult. Duke-Elder (1940) has classified the vascular retinopathies into the following five groups:

(1) Arteriosclerotic retinopathy.

(2) Renal retinopathy.

(3) Toxaemic retinopathy of pregnancy.

(4) Malignant hypertensive retinopathy.

(5) Diabetic retinopathy. 
The same author stresses that it is the exception rather than the rule for these clinical pictures to be present in simple uncomplicated form, since it is usual for one or more types to be combined. The most significant observation which emerges is that all of these pictures are associated with hypertension or arteriosclerosis, or with both together; and the two conditions appear to be so closely related that it is often impossible to determine which is responsible for a particular feature of the fundus picture. This is admitted, in the case of "Angiospasm" by Wagener and Keith (1938), and yet, on this very appearance of "angiospastic retinitis", is based their ocular classification of hypertensive disease which is in general use among physicians and surgeons.

Retinal Haemorrhages and Hard White Exudates.-In cases of fulminating and severe hypertension in reactive vessels, moderately large star-shaped haemorrhages have been observed to arise from a necrotic arteriole (Fig. 22). Apart from this type of lesion, which occurs only in association with high levels of diastolic pressure, all other retinal haemorrhages appear to have a capillary origin.

With the exception of the macular region, the anatomical distribution of the retinal vessels permits the differentiation of the retina in depth into two zones:

(1) The superficial vascularized zone.

(2) The deep avascular zone.

When haemorrhage occurs from the retinal vessels, free blood may be found in either zone. It is rapidly removed from the superficial vascularized zone, but remains much longer in the deep avascular zone where it is forced into the retina, forming large clefts in the cells and fibres of the outer layers.

There are no blood vessels at the macula, but haemorrhages commonly occur from surrounding retinal vessels. The effused blood tends to thrust deeply towards the macula, forming clefts which radiate from the fovea (Fig. 50).

Fig. 50.-Flat preparation of the retina in a case of severe hypertension with reactive sclerosis. In the macular region, superficial haemorrhages follow the nerve-fibre pattern, while deep haemorrhages radiate from the fovea. $\times 27$.

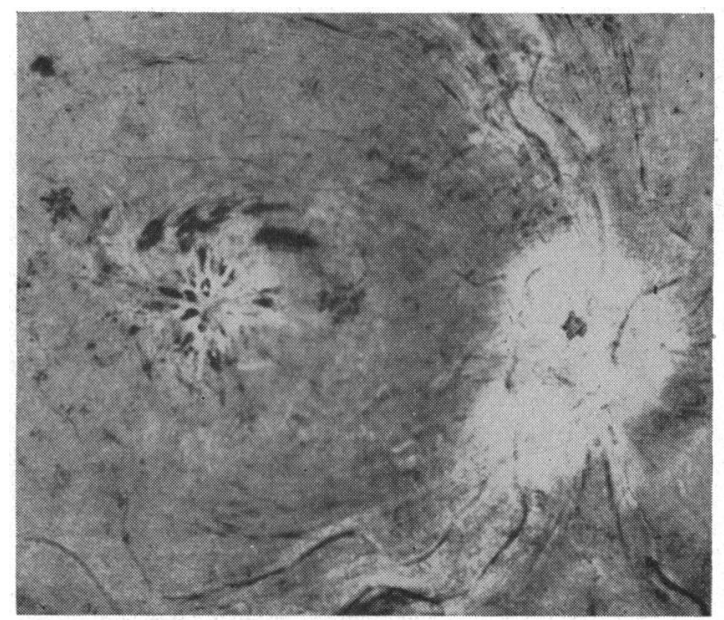


The fate of blood constituents lying in the superficial layers of the retina is uncertain. The red blood cells of a haemorrhagic focus at this level are usually discrete and of normal appearance and give the impression that the haemorrhage is of recent origin.

When blood is effused into the deep avascular layers of the retina, the red cells may be discrete and lie in considerable masses or they may occupy the centre of a mass surrounded by coagulated plasma (Fig. 51). The cell envelopes become distended and the cell substance breaks up into fragments. The envelopes disappear leaving a mass of hyaline fragments and granules which resembles red cells in its staining affinities.

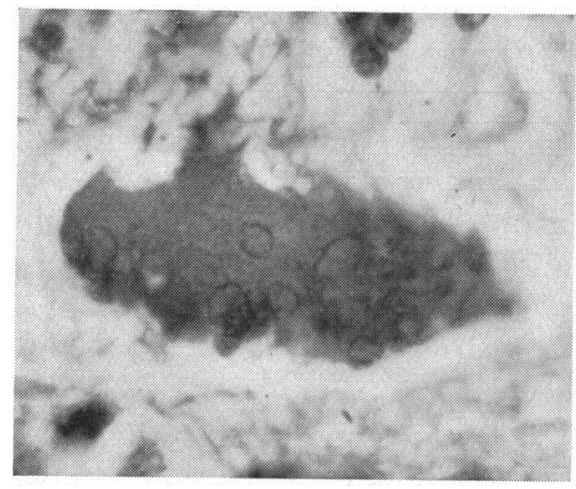

Fig. 51.- Section of retina in a case of severe hypertension. A haemorrhagic effusion in the avascular zone, shows large red blood cells in a mass of hyaline fragments. $\times 600$.

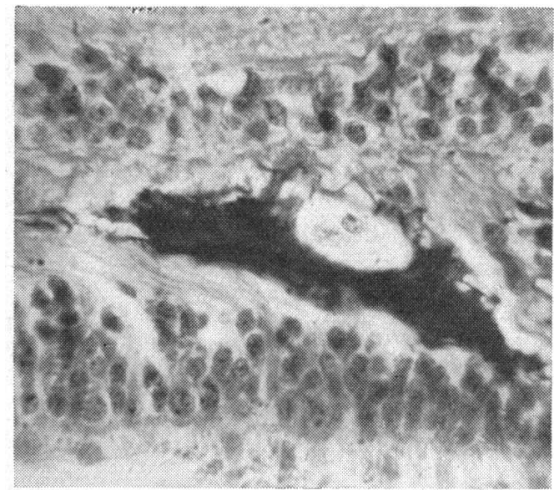

FIG. 52.-Section of retina in a case of severe hypertension. A large phagocyte is seen to be eroding a haemorrhagic mass in the avascular zone. $\times 450$.

Large crescentic erosions are often observed at the edges of the effused masses (Fig. 52), and these cavities are found to be occupied by large phagocytic cells, the nuclei of which resemble those of venous wall cells. The phagocytic cells penetrate deeply into the masses, and, in serial sections, may be shown to form continuous chains connecting the mass to the nearest capillary wall (Fig. 53, opposite). These characteristic phagocytic cells have not been observed within the lumen of adjacent veins or venules, but have been seen lying along the walls of such vessels.

It seems, therefore, that masses of blood effused into the deep layers of the retina are invaded by chains of cells having phagocytic properties. These cells arise from the walls of neighbouring capillaries and venules, and the cells of the chain have nuclei resembling those of venous wall cells. The phagocytic cells do not appear to pass through the vessel walls and enter the blood stream. Presumably the phagocyted material is passed up to the wall of the capillary or vein where it is then disposed of by the cells of the vessel wall. 


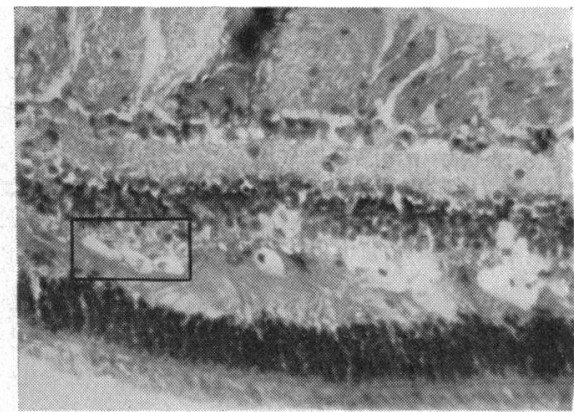

(a) Chains of phagocytic cells lie in the outer layers of the retina. $\times 150$.

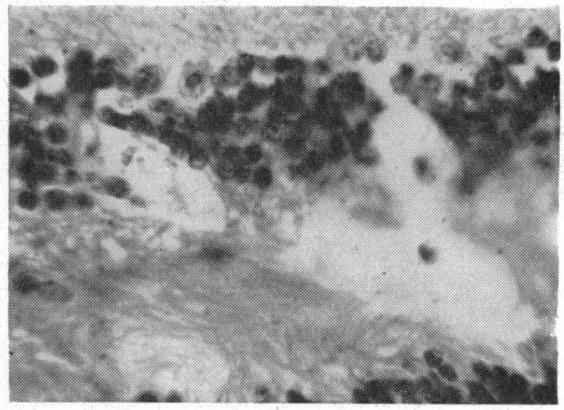

(c) Chains of phagocytic cells lead to the walls of nearby capillaries. $\times 450$.

FIG. $53(a-d)$.- Section of retina in a case of severe hypertension.

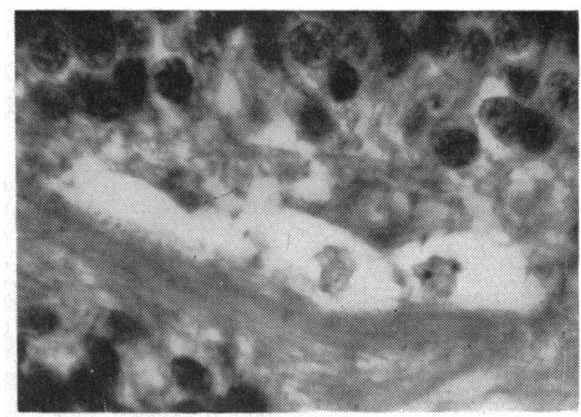

(b) Detail of chain of phagocytic cells shown in $(a) . \quad \times 600$.

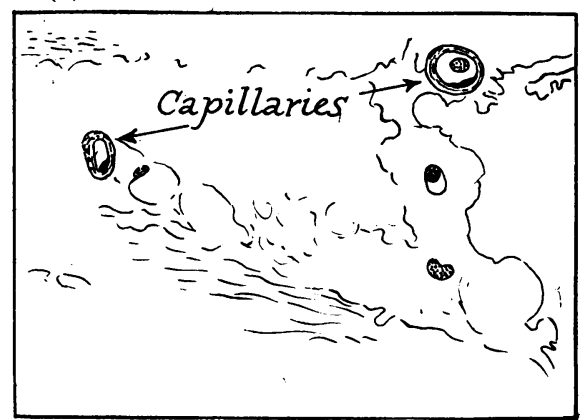

(d) Diagram showing detail of $(c)$.

Case 7, female, aged 37, was an ambulant case of severe hypertension. A fundus photograph was taken, but she did not attend again for repeated observation. 4 months later she was admitted to hospital with intercurrent disease, and died soon after admission. The posterior segment of the eye which had previously been photographed was obtained for histological examination.

The fundus photograph taken in life shows a large mass of superficial and deep haemorrhage near the disc, and beyond this lies a patch of cotton-wool exudate (Fig. 54).

FIG. 54.-Case 7, fundus photograph in a case of severe hypertension, showing a large mass of haemorrhage in the retina.

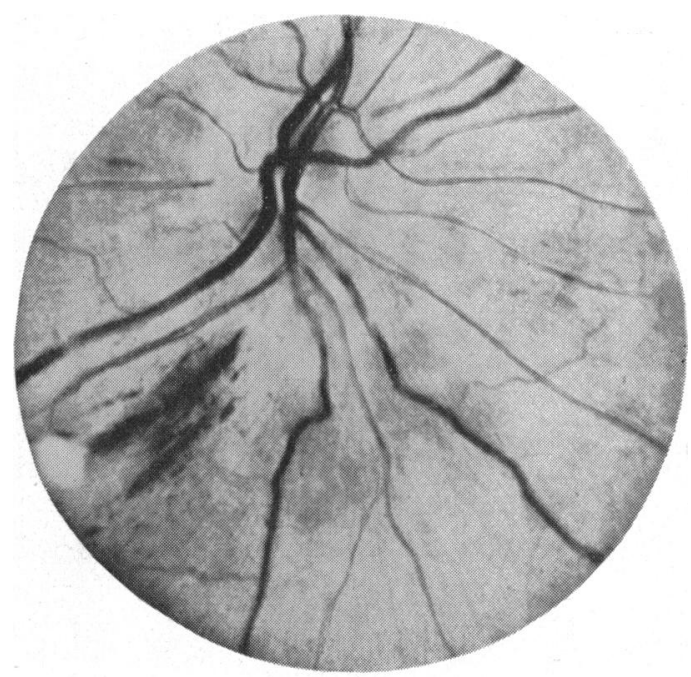


When the posterior segment of the eye was examined macroscopically and by slit-lamp 4 months later, after death, no trace of these lesions could be detected in the retina. When the retinal tissue was laid on the flat there was still no evidence of a lesion, but as the retina cleared in glycerin it was possible only by minute comparison with the photograph taken in life, to detect a slight increase in the density of the retinal tissue of the affected area when it was examined by direct light. There was no evidence of red blood in this area, and there would almost certainly have been no evidence of haemorrhage on ophthalmoscopic examination. When, however, the retina was examined in bulk by transmitted light, it was obvious that the tissues were of abnormal density in the region of the original haemorrhage (Fig. 55); the photograph, however, presents a very much exaggerated picture of the phenomenon. Serial sections were taken through this area. They show deep clefts in the external retinal layers, and these clefts contain the usual masses consisting of coagulated plasma and obvious red blood cells (Fig. 56).

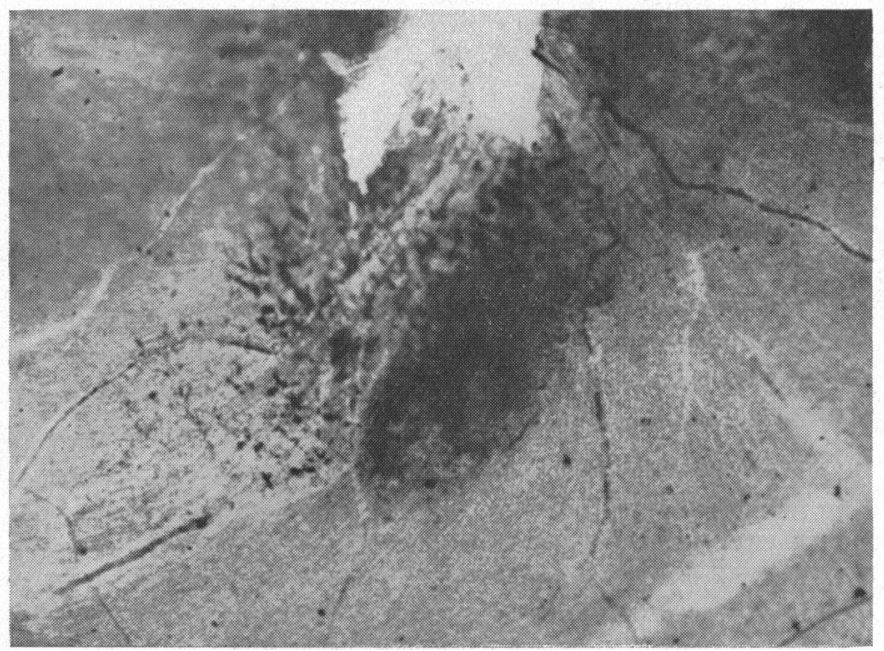

Fig. 55.-Case 7, flat preparation of retina from the same case as shown in Fig. 54. The decolorized haemor- $\varrho \overrightarrow{0}$ rhagic mass is shown by of transmitted light. $\times 40$

FIG. 56.-Case, 7, section of retina taken through decolorized haemorrhagic area shown in Fig. 55. Deep clefts in external layers contain masses of coagulated plasma and red blood cells. $\times 129$.

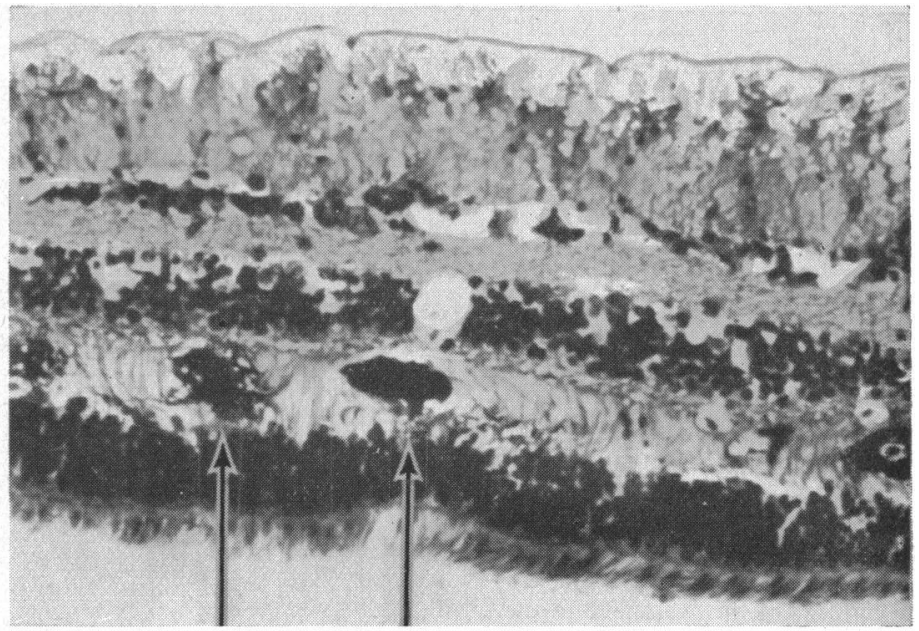


It appears that masses of effused blood lying in the deep avascular layers of the retina are soon decolorized, and may not be recognizable with the ophthalmoscope, although, after staining, they still appear as characteristic red cells in histological section. These invisible masses in the avascular layers of the retina are then slowly eroded and cleared away by chains of phagocytic cells arising from the nearby venules and capillaries of the superficial vascularized retinal layers. It is probable that the small, round, hardedged white exudates seen in the retina are ophthalmoscopic evidence of phagocytosis of old decolorized effusions lying in the deep layers of the retina.

In the case shown in Fig. $57(a-c)$, a large mass of haemorrhage was present in the retina; 4 months later the haemorrhage had disappeared but there, was some disturbance of retinal pigment in the region it had occupied. After a further 7 months, hard white exudates were observed throughout the same area.

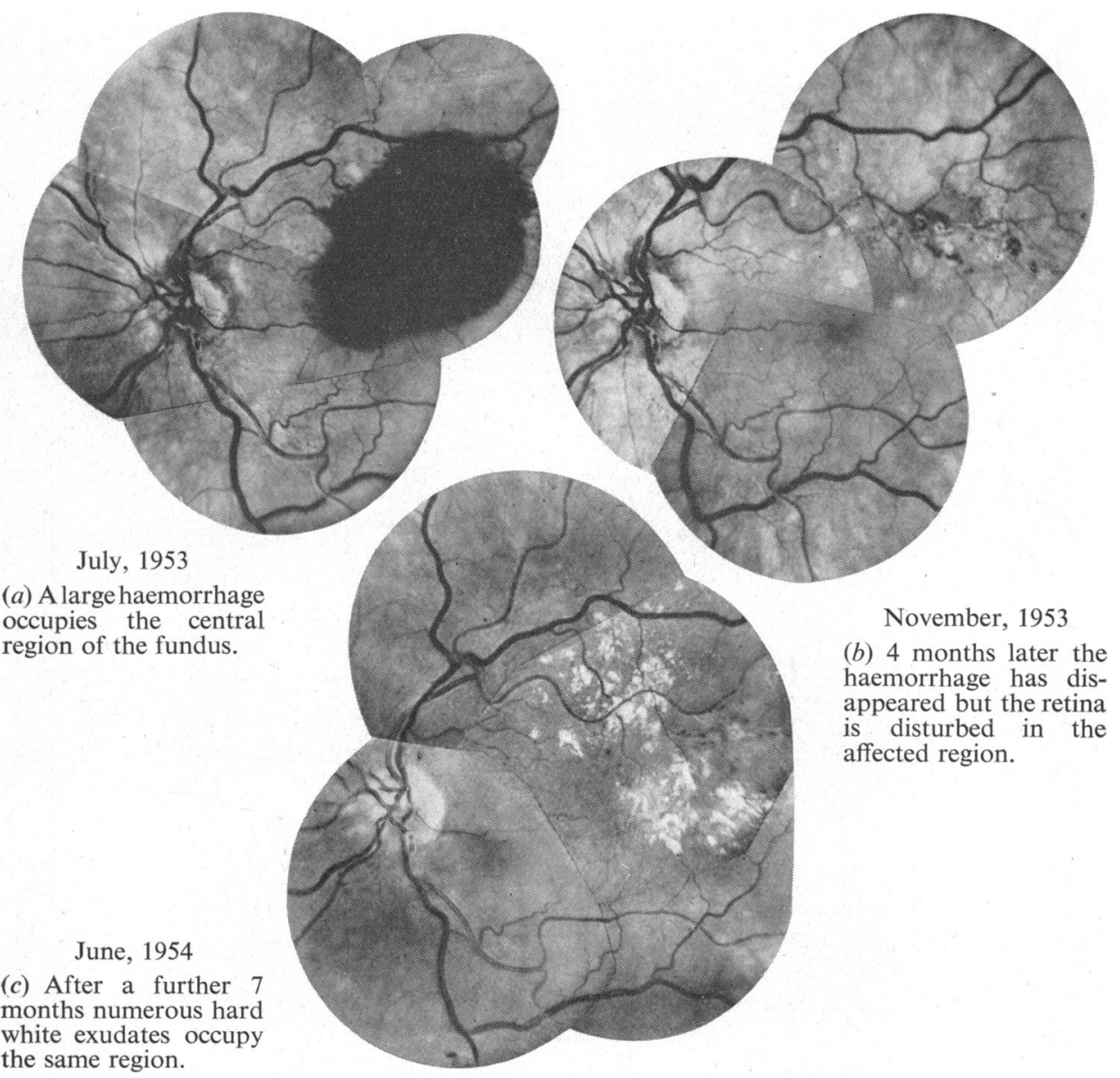

FIG. $57(a-c)$.- Serial photographs in a case of severe hypertension with reactive sclerosis. 
Soft White Exudates.-Large, soft-edged, cotton-wool exudates are a feature of both severe and fulminating cases of hypertension. They appear suddenly in the superficial layers of the retina, obscuring the vessels, when the arterioles are of young and reactive type, and the diastolic pressure is 130 $\mathrm{mm}$. $\mathrm{Hg}$ or more. Repeated observations show that they clear rapidly, often disappearing without trace in 4 to 6 weeks, and that they are always related to a small thrombotic arteriole (Fig. 58, $a-d$ ).

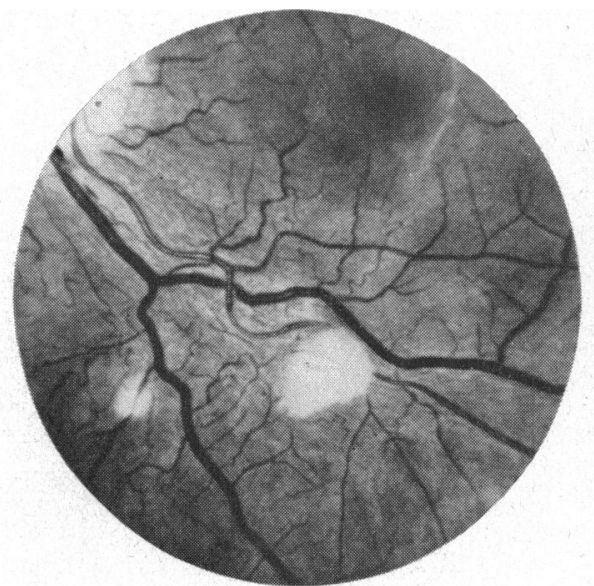

(a) January, 1953

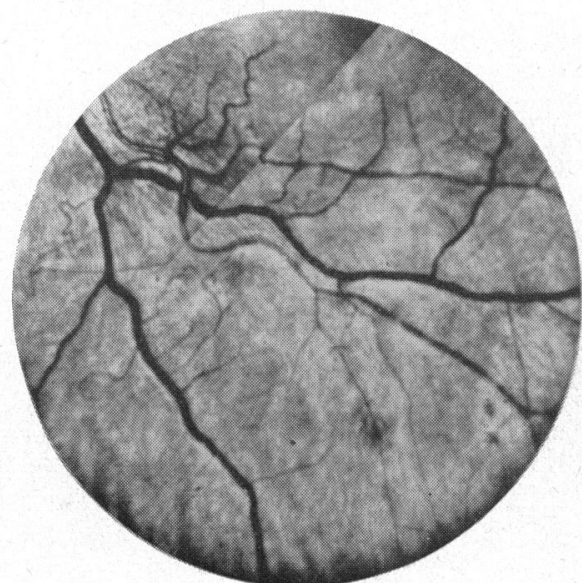

(c) March, 1953

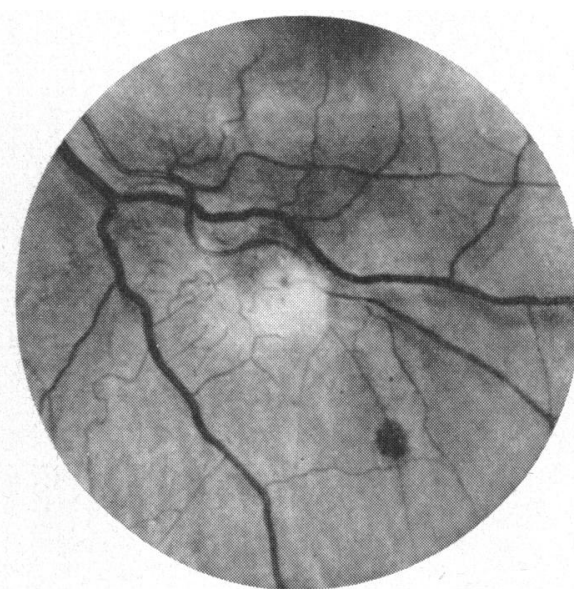

(b) February, 1953

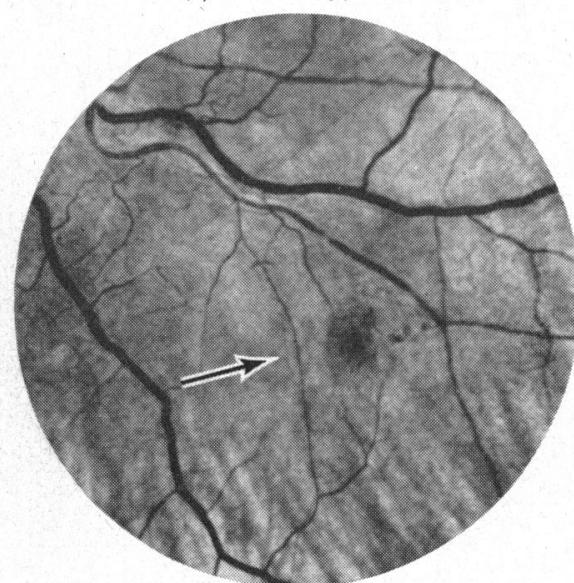

(d) December, 1953

FIG. $58(a-d)$.- Serial fundus photographs in a case of severe hypertension with reactive sclerosis. A patch of cotton-wool exudate, lying superficial to the retinal vessels, is seen to clear during a period of 6 weeks. A thrombotic arteriole, hidden by the exudate, becomes visible as the patch clears, and in $(d)$ it is seen to anastomose with a nearby venule.

It appears therefore, that the cotton-wool patch in severe cases of hypertension is the result of sudden occlusion of a small arteriole; in other words, it is a pale infarct. The clearing of the cotton-wool patch is analogous to the clearing of the opaque retina after severe occlusion of the central artery. 
After the cotton-wool patch has cleared from the retina, marked lability may be observed in the small vessels of that area. In Fig. $58(d)$ the circulation has become re-established in the arteriole which was previously thrombosed, and it is seen to anastomose with a retinal venule. Fig. 59 shows a region of the retina initially supplied by an arteriole with four terminal branches; a cotton-wool patch suddenly appeared obscuring the vessels, and as the small arterioles again became visible after clearing of the patch, all of them appeared to be thrombosed. The two inner ones subsequently disappeared, and the two outer ones became incorporated into the local circulation as a venous loop.

FIG. 59.-Fundus photograph in a case of severe hypertension with reactive sclerosis. A small arteriole is seen to arise by a fine white thread from the parent vessel. The distal portion of the arteriole is now functioning as a small vein.

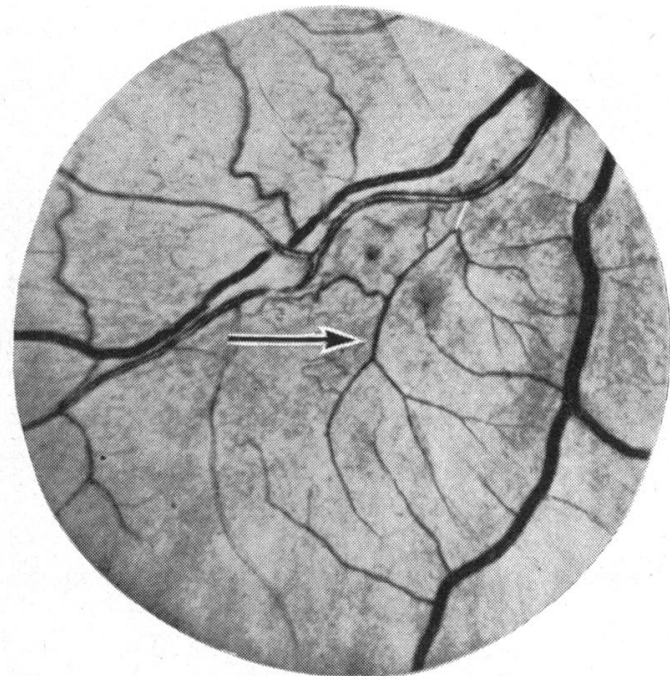

In serial sections of retinal tissue in cases of severe and fulminating hypertension, a patch of cytoid bodies is often found (Fig. 60). The nature of the histological changes responsible for this appearance has been much disputed. The method of serial section has brought into prominence the fact that such a patch of cytoid bodies is always related to a small arteriole in a state of necrosis (Fig. 61). The nearest venules are usually deeply congested. The

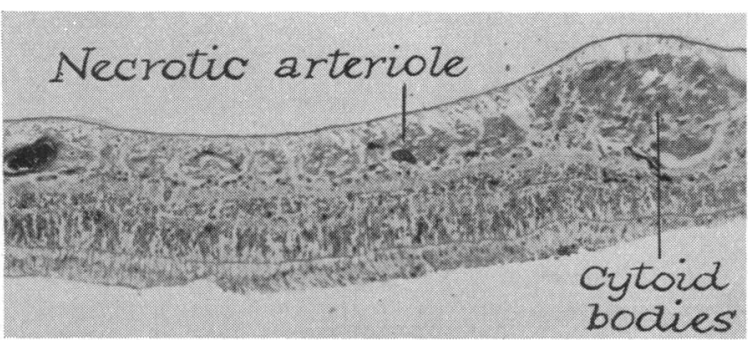

FIG. 60.-Section of retina in a case of severe hypertension showing a patch of cytoid bodies related to a small necrotic arteriole. $\times 60$.

FIG. 61.-Section of retina showing detail of necrotic arteriole seen in Fig. $60 . \quad \times 400$.

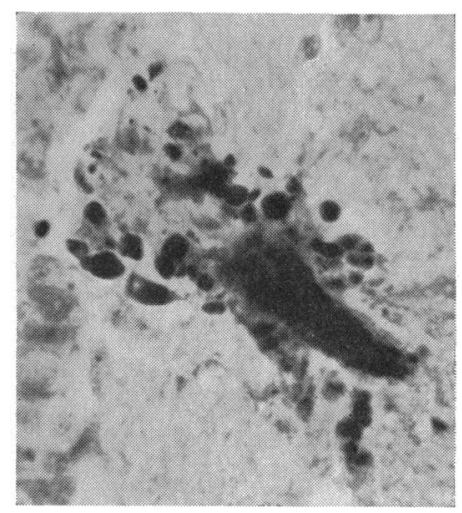


size of the patches of cytoid bodies, their superficial situation, their frequency distribution in the retina, and their constant relation to an occluded small arteriole, have led to the hypothesis that they are the histological counterparts of the cotton-wool patches seen in life.

If superficial cotton-wool patches are due to arteriolar necrosis, and some of them almost certainly are, then it is important to note that their occurrence is not confined to cases of fulminating hypertension, but that they may appear in any case of severe hypertension with reactive vessels in which the diastolic pressure reaches $130 \mathrm{~mm}$. $\mathrm{Hg}$ or more. Thus, post-mortem evidence of arteriolar necrosis is not necessarily pathognomonic of malignant hypertension as is held by some authorities on pathology. Moreover, the absence of cotton-wool patches, even at diastolic pressures of $140 \mathrm{~mm} . \mathrm{Hg}$, in cases of senile involutionary sclerosis with hypertension, is further evidence that the vessels in such cases are non-reactive.

Thus, the features known as retinopathy result from changes which are either reparative or degenerative in nature. The veins appear to mediate the function of repair, since the retina has no lymphatic vessels. The factors of sclerosis and hypertension seem to be concerned in the production of vascular degeneration, and breakdown is seen to occur in two different ways.

(a) Youthful reactive vessels, subjected to mounting diastolic blood pressure, become affected by arteriolar necrosis and thrombosis, causing ischaemic lesions and effusions in the retina, lesions which are prone to occur when the diastolic pressure reaches levels of $130 \mathrm{~mm}$. $\mathrm{Hg}$ or more.

(b) Fibrotic elderly vessels of involutionary type, and even those with reactive fibrosis, can contain diastolic pressures of $140 \mathrm{~mm}$. $\mathrm{Hg}$ without degenerative breakdown; but such vessels seem to have a reduced range of safety with regard to pressure lability and appear to suffer atheromatous occlusion, with retinal ischaemia and effusions, if the pressure levels to which they are adapted become significantly reduced.

Arteriosclerotic Retinopathy.-This appears to depend primarily on the attainment of a state of fibrosis in the retinal vessels. This will usually be reached in elderly people through involutionary sclerosis. Extensive sclerosis eventually results in difficulty in maintaining an adequate pressure and volume of blood in peripheral vessels. The advent of benign hypertension in many cases will operate to keep the rigid vessels well filled, but will also tend with the passage of time to increase their rigidity. When decompensation occurs, for some reason or another, such as the fatigue which follows excessive exertion, the change from an active to a sedentary habit, or perhaps even injudicious hypotensive therapy, a relative diminution in peripheral pressure and volume will ensue, leading to partial atheromatous occlusion. If the peripheral pressure and volume are quickly re-established, the lesion may be reversible, but, if recovery is delayed, the lesion is permanent. In the distal portions of the vessel, fibrosis and low pressure lead to 
further occlusive lesions. Retinal ischaemia, capillary haemorrhages, and their clearing by phagocytosis follow, and the composite picture is that of arteriosclerotic retinopathy.

Much less frequently the same picture can be reached by another route. Moderate, but persistent, hypertension in youthful vessels, would ultimately lead to diffuse reactive sclerosis and advanced rigidity; such a condition could only be attained pari passu with increased blood pressure, and if it did not remain fully compensated would result in occlusive lesions and retinopathy.

Fulminating Hypertensive Retinopathy.-A strong hypertensive stimulus occurring in youthful vessels causes a marked reactive response in the arteriolar wall. Cells increase in number and lay down new elastic fibres at all levels of the thickened wall surrounding a narrowed lumen. Rising diastolic pressure is opposed by constricting forces in the reactive wall, and causes acute necrosis in the cells of the wall, with consequent damage to the endothelium and arteriolar thrombosis; dissecting aneurysms may occur in the vessel wall, which may even rupture. Acute arteriolar occlusion causes pale infarcts in the retina, which appear as patches of cotton-wool exudate. Haemorrhagic effusions from the dilated capillary bed frequently occur, and, in process of clearing by phagocytosis, produce patches of hard white exudate. The retinal oedema has a clear quality suggesting transudation. In these cases the retinopathy does not appear till diastolic pressures of about $130 \mathrm{~mm}$. $\mathrm{Hg}$ have been reached. The whole picture suggests an acute degenerative process due to high pressure, and the ophthalmoscopic features indicative of arteriolar fibrosis are absent from the vessels.

Renal Retinopathy.-Transient hypertension associated with acute glomerulo-nephritis in children does not produce renal retinopathy; nor does the hypertension which occurs in acute glomerulo-nephritis in adults. On the other hand, a history of previous acute glomerulo-nephritis is often obtained in cases showing a fundus picture of severe hypertension with reactive sclerosis. Such cases do not, however, present the typical features of advanced renal retinopathy; the fundus appearances indicate no more than severe hypertension with incipient reactive sclerosis in vessels of youthful type. Cases of nephrosis in children present a characteristic fundus picture; the arterial pattern is normal, but the veins are markedly dilated, tortuous, and cyanotic, and the background appearances suggest low-grade oedema of the disc and retina; there is no hypertension and no retinopathy. Again, in a case of total anuria lasting 23 days, before a fatal termination, associated with severe nitrogen retention but without hypertension, the fundus was examined on the 23 rd day and was found to be normal in all respects; there was no amaurosis in this case. Thus, hypertension appears to be necessary for the production of renal retinopathy, but is not the sole factor responsible for its appearance.

Elevation of blood pressure is often early and transient in Type I nephritis, 
but in approximately 10 per cent. of cases there is continued hypertension with reactive sclerosis which may ultimately proceed to an acute retinopathy of hypertensive rather than of renal type.

Type II nephritis is typically without hypertension, unless in the late stages, and then only in some cases. It is in these cases that typical renal retinopathy is observed. The mode of degeneration is acute, with papilloedema, oedema of the retina, numerous cotton-wool patches, haemorrhages, and hard white exudates featuring in the picture. The oedema has an opaque appearance suggesting a plasmoid quality, and the features of acute vascular breakdown usually occur at lower levels of diastolic pressure $( \pm 115 \mathrm{~mm}$. $\mathrm{Hg})$, than in cases of fulminating or severe hypertensive retinopathy $( \pm 130$ $\mathrm{mm} . \mathrm{Hg}$ ). It may be that, while factors such as nephrosis and severe nitrogen retention cannot alone produce retinopathy, they may lower the threshhold for, and increase the severity of, acute hypertensive degeneration.

Two cases of acute retinopathy in children, observed concurrently, present interesting and contrasting features:

Case 8, girl, aged 10 (Fig. 62). Pyelonephritis of one kidney. Specimens of urine from the opposite ureter were normal. The diseased kidney was removed and found to have a bifid ureter. Only one branch and its related renal tissue was affected by pyelonephritis. Hypertension persisted after operation and reached high levels (B.P. 260/170), although specimens of urine from the remaining kidney were still normal. Death occurred in terminal uraemia 20 months after the onset of the symptoms. The course of the illness may have been prolonged by treatment with hexamethonium bromide.

The fundus picture was one of fulminating hypertension when first seen, about one year from the onset of symptoms. The background was pale and the normal brilliance of the child fundus was lacking. The retina was oedematous along the course of the main vessels; the disc was grossly oedematous, but the oedema had a clear quality which did not obscure the small vessels on its surface. The arterioles were diffusely pale, and although sometimes approaching normal calibre, were, in general, diffusely narrow; they were unexpectedly sinuous for a hypertensive child, and the loops occupied all three dimensions of the retinal space. Portions of the arteriolar walls were thickened and less translucent (hyperplasia of the wall), and in these portions the blood column was even more pale, narrowed, and finely variable in calibre. Broad parallel zones of concealment were observed where these portions crossed a vein. The veins distal to such crossings were congested. The arterial branchings were wide-angled and back-curving. Small, round, hard-edged, white exudates were numerous around the disc, and in the central area where there was a well-defined macular star. Many small haemorrhages were present and were seen to come and go during a year's observation.

Case 9, girl, aged 10 (Fig. 63). Pyelonephritis was bilateral from the beginning. Death occurred in uraemia 3 years later. Hypertension was severe for her age but the diastolic level was never over $115 \mathrm{~mm}$. $\mathrm{Hg}$.

The fundus picture was drawn about one year before death and is therefore comparable with the previous case. It presents certain contrasting features. 


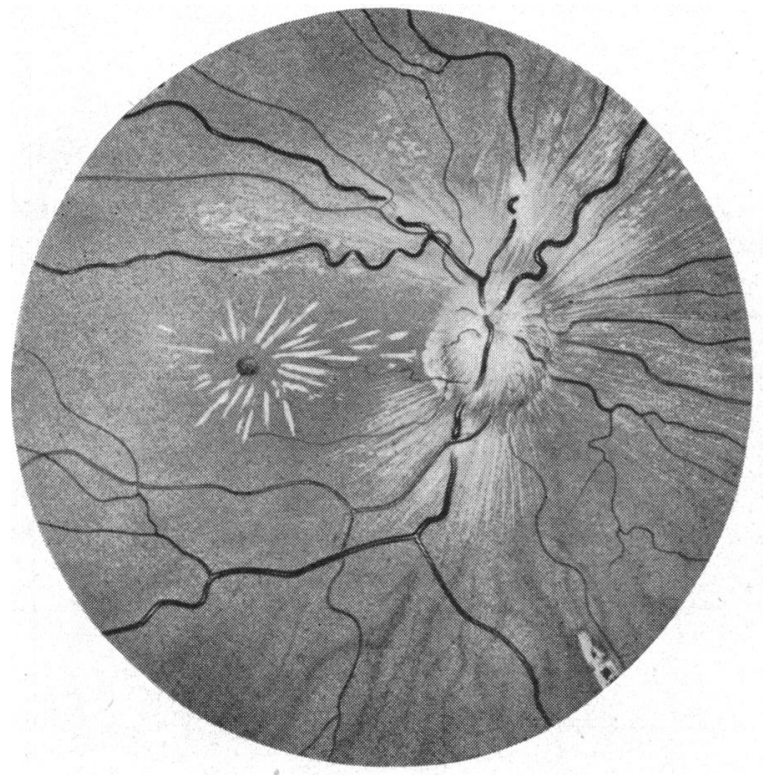

Fig. 62.-Case 8, drawing of fundus in a case of fulminating hypertension in a child. The disc and retina are oedematous; the oedema has a clear quality which does not obscure the small vessels. The arterioles are diffusely pale and narrow, but are unexpectedly sinuous for a hypertensive child. Broad zones of concealment are shown at some of the arterio-venous crossings, and the veins are congested distal to the crossings. Hard white exudates are numerous.

FIG. 63.-Case 9, drawing of fundus in a case of renal retinopathy in a child. The disc and retina are oedematous; the oedema has a cloudy quality which obscures the vessels in places. The arterioles show regions of fibrosis, in addition to hyperplasia and hypertonus. Concealment and congestion of veins are present. Cotton-wool exudates are numerous.

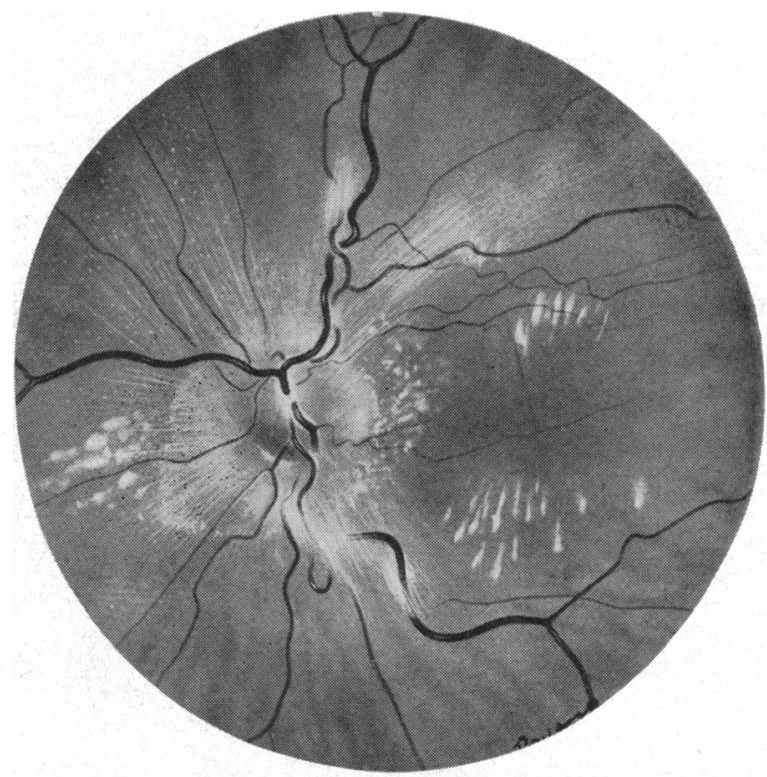

There was gross oedema of the disc and some oedema of the retina, but in this case the oedema was of a cloudy nature. Hard-edged, white exudates were minimal, but patches of opaque "cotton-wool" exudates were frequent. In many places the vascular pattern was obscured by oedema or exudate which prevented consecutive study of a vessel along its length. Proximal portions of the artery, however, were seen to be red and wide (fibrosis); other portions in the mid-zone were markedly pale, narrow, and finely variable in calibre, and showed broad 
bands of concealment at crossings (hyperplasia); distal portions were pale, straight, and narrow (hypertonus). Veins were congested distal to arterio-venous crossings.

The first case was regarded as one of fulminating hypertension of renal origin in which renal failure was but a brief terminal episode. The appearances of the arterioles suggested hypertonus and hyperplasia, but the high levels of diastolic pressure had produced arteriolar degeneration before there was time for significant fibrosis.

In the second case renal disease dominated the picture and was complicated by severe hypertension. Very high levels of diastolic pressure were never recorded, and the retinal arterioles showed long segments of fibrosis in addition to other portions showing hyperplasia and hypertonus. The appearance of the retinal oedema suggested a "plasmoid" quality which contrasted with the "serous" quality of the former case.

Toxaemic Retinopathy of Pregnancy.-The fundus pictures of hypertension which have been observed in pregnant women do not differ from the pictures of hypertension occurring in the general population; they fall into the same two basic groups:

(1) Benign hypertension in prematurely fibrotic vessels.

(2) Potentially severe hypertension in young vessels.

The prognosis with regard to hypertension in the former group appears to be good, and in the latter group it appears to be more favourable than usual, since the termination of pregnancy may diminish or remove the hypertensive stimulus. At the same time, however, pregnancy appears to be a fruitful exciting cause of severe hypertension with reactive sclerosis in later life.

If retinopathic degeneration occurs, it is of acute type and, as in renal retinopathy, severe breakdown tends to appear at relatively lower levels of diastolic pressure than in fulminating hypertensive retinopathy.

Several cases of transient complete amaurosis of pregnancy have been examined during the amaurotic phase and, in all cases, the retinal arterioles were found to be of normal appearance. There was no evidence of hypertonus; and it is probable that the amaurosis is of central origin in these cases. Similarly, in a case of transient complete amaurosis due to quinine poisoning, the retinal vessels were normal during the amaurotic phase.

Diabetic Retinopathy.-Diabetic patients, without retinopathy but with hypertension, show the fundus pictures of hypertension. But when retinopathy is present in hypertensive diabetic subjects, the hypertensive and arteriosclerotic features described above are so altered that classification of the fundus picture from these aspects is unreliable. This is a stimulating finding, since diabetic retinopathy is so far the only condition in which the observed picture has shown no relation to the expected picture. 


\section{DisCUSSION}

\section{General Considerations}

It is currently held that the state of hypertension depends upon increased peripheral resistance, and that this in turn is due to generalized arteriolar hypertonus. It seems to be tacitly assumed by most authors that the arterioles are universally capable of diffuse increase in tonus. This conception, based on animal experiments which take no account of arteriosclerosis, has been carried into the fields of experimental and clinical medicine. It has been shown that arteriosclerosis does not cause hypertension, but the possibility that it may play a part in modifying the course of the disorder appears to have been disregarded. Consequently, while much attention has been paid to studying and attempting to classify the behaviour of the arterial blood pressure, little consideration appears to have been given to an equally important factor, the condition of the arteriolar wall with regard to sclerosis.

Normal ageing processes in the walls of arteries are either omitted or dismissed too lightly by most authorities on the subject of pathological arteriosclerosis. Allbutt (1915) clearly stressed the difference between involutionary and reactive sclerosis in the clinical field, but his distinctions have been largely unheeded to the present day. With the exception of Monckeberg's sclerosis, which is a gross disorder involving calcification of the media, the types and intensity of sclerosis in the vascular tree are difficult to estimate by clinical methods, and this may be why the sclerotic element is usually disregarded in the medical investigation of hypertensive cases.

Current medical classifications of hypertension are usually based on aetiological factors. Cases secondary to renal, endocrine, and other disorders are satisfactorily segregated, leaving a large group of cases termed primary essential hypertension. The cases within this group are far from homogeneous, but further classification has proved difficult. A few cases run a fulminating course, but the remainder, designated benign essential hypertension, show great variation in the course and severity of the illness. The present studies appear to elucidate some of these anomalies, and indicate that much of the difficulty now being experienced in classifying hypertensive disease is due to failure to appreciate the arteriosclerotic factor. For instance, two patients may have identical blood pressure levels, say 250/140 $\mathrm{mm}$. $\mathrm{Hg}$, and yet present completely different clinical pictures. The younger patient may be desperately ill; fundus shows severe hypertension with reactive sclerosis, fibrosis is minimal, and acute degenerative breakdown is wellmarked. The other elderly patient may show advanced involutionary sclerosis, with hypertension but without retinopathic breakdown, and yet be apparently well and resentful at being confined to hospital.

The combined ophthalmological and medical classification of Wagener and Keith (1937), which is in general use amongst physicians and surgeons at the present time, depends on the recognition of the presence or absence of 
angiospasm in the retinal arterioles. In the course of the present work there has been clinical evidence that hypertonus persists during phases of high blood pressure, and disappears in transient cases after the pressure falls, but there has been no evidence to suggest that sudden extreme changes, such as are implied in the use of the term "angiospasm", occur in the retinal arteries in hypertensive disease. The classification of hypertension and arteriosclerosis made by Duke-Elder (1940) integrates the theoretical with the ophthalmoscopic data, and divides the known clinical pictures into two main classes-vascular sclerosis and vascular retinopathy. The indications arising from the present studies are in broad general agreement with the theoretical aspect of this classification, although differing in the delineation and interpretation of the clinical detail of the various types of fundus picture.

The ophthalmoscopic method of classifying hypertensive and arteriosclerotic cases described in the preceding pages has been applied in routine clinical work during the past 5 years. It has proved to be practicablein most cases, with regard both to the recognition of the hypertensive state, and to the grouping of cases with relation to arteriosclerosis. The method shows a high degree of correlation with clinical blood pressure readings, but differentiation on aetiological grounds into primary essential and secondary hypertension cannot be made with the ophthalmoscope. The fundus pictures have been seen to fall into seven main groups and, although interchange of cases between some of the groups has been observed, time will be required before the details of these transitions can be established. For instance, the sequence from involutionary sclerosis (Group 1) into involutionary sclerosis with hypertension (Group 2) has not yet been observed. Group 2 cases have, however, been seen to change into advanced involutionary sclerosis with hypertension (Group 3). Interchange has frequently been observed between the appearance in normal youth (Group 4) and that in diffuse hypertonus (Group 5). The hypertonic fundus picture (Group 5) has not yet been seen to change into fulminating hypertension (Group 6), although in a few cases partially controlled by hypotensive therapy, Group 6 cases have been observed to change into severe hypertension with reactive sclerosis (Group 7). The development of Group 7 cases from Group 5 cases is common, and it is possible that a few cases from Group 7 may survive to develop a fundus picture closely similar to that seen in Group 3, although such a transition has not yet been identified.

It has been stated by ophthalmologists, and is currently accepted, that the most constant and characteristic sign of hypertension is a straightening and narrowing of the retinal arterioles. But this is the normal appearance in elderly subjects with involutionary sclerosis uncomplicated by hypertension. In young persons, in both early and severe cases of hypertension, the retinal arterioles are certainly abnormally straight and narrow, but these cases are not very numerous. The reverse is the rule in involutionary sclerosis with hypertension, the most benign and common form of high blood pressure. 
The striking feature in this condition is that the retinal arterioles are unexpectedly wide and curvilinear in their more fibrous portions when viewed against an aged fundus background.

The question whether "malignant" hypertension is a separate type or merely a phase of essential hypertension is still disputed. The presence of papilloedema is usually regarded as one of the chief criteria by which a diagnosis of malignant hypertension may be made. It has been seen that papilloedema may occur in cases of partial atheromatous occlusion of the central retinal artery in cases of hypertension associated with fibrosis. The following sequence of events can readily be conceived in a case of severe hypertension with reactive sclerosis. After a course of several years, the fundus picture will show fibrosis, hyperplasia, and hypertonus in different parts of the same retinal arteriole, together with occasional patches of cottonwool exudate resulting from arteriolar necrosis. Finally, papilloedema may occur with increased retinal haemorrhages, giving the impression that the condition has entered a malignant phase. It is possible, and even probable, that the advent of papilloedema in cases showing established fibrosis in the retinal arterioles is to be interpreted merely as evidence of partial atheromatous occlusion of a fibrotic central retinal artery. True fulminating or "malignant" hypertension is a relatively rare form of hypertension occurring in a young vascular system, and papilloedema is always an early feature; the retinal arterioles do not have time to compensate beyond the stage of hyperplasia before acute arteriolar degeneration appears, and they do not show ophthalmoscopically the features of fibrosis.

\section{THERAPEUTIC CONSIDERATIONS}

It is axiomatic, in hypertensive disease as in other illnesses, that if the cause of the condition can be found it should be removed. Unfortunately, this course is seldom applicable. In most cases of hypertension the problem is one of management, and a choice must be made between alternative lines of treatment. The more reasoned and accurate the diagnostic grouping of cases, the more rational will be the selected regime.

If we assume the validity of the main hypothesis and the ophthalmoscopic grouping of cases resulting from this hypothesis, certain therapeutic principles become apparent. Benign hypertension, in the strict sense of primary involutionary sclerosis with hypertension, is predetermined by the presence of arteriolar age-fibrosis before the onset of hypertension. The patient is partially protected by his fibrotic vessels, which limit the severity of the increase of peripheral resistance and the levels of diastolic pressure attained in the hypertensive state. The arteries and the arterioles are partially degraded by relative fibrosis, and to this extent have lost reactivity and are proportionately less likely to undergo acute degenerative change. Very high diastolic pressures $( \pm 170 \mathrm{~mm}$. $\mathrm{Hg})$ are not observed in this group, and high diastolic pressures $( \pm 130 \mathrm{~mm}$. $\mathrm{Hg})$ do not have the same proclivity to cause 
arteriolar necrosis; relatively low but raised diastolic pressures $( \pm 115 \mathrm{~mm}$. $\mathrm{Hg}$ ) are the rule, but do not carry any serious prognostic significance. On the other hand, so long as the heart muscle is sound, all these benign cases show elevation of the systolic pressure, and this may reach very high levels.

The fundamental weakness in the vascular physiology of cases of involutionary sclerosis is the difficulty of maintaining adequate peripheral circulation in vessels which are relatively rigid. Hence, slight degrees of systemic hypertension may even be beneficial to the involutionary sclerotic patient provided the pressures are fairly constantly maintained. While the upgrading of the systemic pressure resulting from hypertension appears to be well tolerated by subjects of sclerotic type, it seems to reduce the safe range of pressure lability in such persons, as is evidenced by their proclivity to atheromatous degeneration if the pressure, for some reason or other, is again downgraded. It has also been shown that continued moderate elevation of the diastolic blood pressure is associated with thinning of the walls of the arterioles where they are affected by involutionary sclerosis. Such vessels stand up well to continued high levels of systolic pressure, but can be conceived to rupture readily in the presence of a sudden systolic "tidal wave".

This group accounts for the majority of hypertensive patients. Since they appear to sustain the usual slight elevations of pressure without harm, active measures to promote hypotension seem to be contraindicated and may even be dangerous. At the same time, these patients must be protected against sudden significant elevations of systolic pressure. Over-exertion and excessive fatigue should both be avoided, and the patient should be encouraged to live actively a life of moderation in all things. Even rest should be taken in moderation for a short period daily, and prolonged periods of rest in bed are best avoided. Mild sedation may be required to control nervous or physical over-activity, but hypotensive drugs are contraindicated; operative surgical measures designed to reduce pressure may be dangerous. In this connexion it is well to remember that although the mean age of these cases falls in the decade of 60 to 70 years, the condition of primary involutionary sclerosis with hypertension has been observed in patients less than 30 years old.

Symptoms are seldom pressing in this group, and may be alleviated by simple measures as they arise. Diplopia is usually transient under a regime of moderate activity and mild sedation. Cases of partial arterial occlusion appear to respond much better to moderate activity than to prolonged rest in bed. The latter course may prove catastrophic to the eye as the result of further diminution of the peripheral circulation.

"Malignant" hypertension is much more rare than the occurrence of papilloedema in hypertensive subjects, and the main differentiating feature in such cases is the absence of arteriolar fibrosis. If, as is usually the case, the source of hypertension cannot be eradicated, the main indication, imperative in its urgency, is to control the pressures at such a level that they cease to be a danger, and to permit time for the arterioles to undergo compensatory 
reactive changes. When first seen, the patient is often extremely ill. Rapidity of control is essential, and complete rest in bed is a simple step in this direction. The choice of treatment then lies between vascular surgery and medical control by prolonged rest and hypotensive drugs. It is not within the province of the ophthalmologist to adjudicate between those alternatives; success has resulted from either course. It is legitimate, however, and may be helpful, to mention some of the theoretical principles which appear to influence the type of case known as fulminating hypertension.

In order to attain and contain such pressures as $\pm 260 / \pm 160 \mathrm{~mm}$. $\mathrm{Hg}$, the arterial system must in general, and in the first instance, be of youthful type. It is well known that young persons living an active outdoor life may suffer from very localized atheroma and rigidity of the aorta and its deep branches. Theoretically, there may be a large localized plaque of atheroma in a main renal artery near its origin, while the peripheral vascular system is young and healthy. A main renal artery may thus be locally rigid in malignant hypertension, and may therefore be predisposed to the development of atheroma as a result of falls in pressure. Such a patient might well appear to benefit in the first instance from operation or hypotensive therapy, only to relapse on account of the establishment of a vicious cycle excited by renal ischaemia. The fundus picture would not help in the segregation of such cases. The prognosis in untreated fulminating hypertension is so bad, however, that measures designed to reduce the pressure rapidly should not be withheld, provided there is no indication of vascular fibrosis in the retina or elsewhere. Recovery with complete reversal of the fundus picture has been observed in cases in which a firm diagnosis of fulminating hypertension had been made on a basis of the general medical and ophthalmological features. Some of these patients have been well enough to return to work as a result of intensive hypotensive therapy and subsequent control of blood pressure. In other cases the treatment has sufficed only to reduce the pressure to safer levels. The fundus picture has changed from one of fulminating hypertension, with arteriolar hypertonus and hyperplasia, to one of severe benign hypertension with the appearance in the arterioles of long segments of fibrosis in addition to hyperplasia and hypertonus; these cases, however, seem to have a reduced range of safety with regard to pressure lability, and are prone to develop atheromatous occlusion.

Severe hypertension is regarded as synonymous with continued hypertension with secondary arteriosclerosis. These cases are benign in so far as time to develop arteriolar fibrosis can be allowed, but they are severe in that initially the arterioles are sufficiently young to produce moderately high levels of diastolic pressure $( \pm 130 \mathrm{~mm} . \mathrm{Hg})$. They are severe because the arterioles are reactive and liable to become necrotic or thrombotic at moderate diastolic pressures; yet their course is not fulminating and malignant because they develop sufficient fibrosis to determine moderate, rather than very high, diastolic pressures. Hypertension of pregnancy and renal disease appear 
with suggestive frequency in the aetiology of this group. The principles of treatment for such cases are more difficult to define. The presence of arteriolar fibrosis weighs as a contraindication to extreme measures of hypotensive therapy. At the same time it is desirable to control the diastolic pressure at approximately $120 \mathrm{~mm}$. $\mathrm{Hg}$. This is probably below the necrotizing level, yet not so low as to precipitate atheromatous occlusion. Maintenance of the diastolic pressure at a safe level should allow time for further protection by replacement fibrosis. Prolonged inactivity in bed seems inadvisable, if it can be avoided; many cases prosper as ambulant patients on a regime of moderate activity, combined with the exhibition of hypotensive drugs in the minimum dosage necessary to control the diastolic pressure at a safe level. The indications with regard to treatment may therefore be summarized as follows:

(1) To reduce the diastolic pressure below the level at which high pressure degenerative lesions may occur.

(2) To prevent the diastolic pressure falling to a level at which low pressure degenerative lesions may occur.

(3) To gain time for further protection by secondary replacement fibrosis.

\section{SUMMARY}

Cases of hypertensive disease may be recognized and classified by the fundus appearances into two main groups:

(1) Those associated with retinal evidence of senile or premature involutionary sclerosis;

(2) Those without such features.

In the first group, hypertension occurs in vessels already largely defended by involutionary sclerosis. The fibrotic portions of the vessels become dilated with increased pressure, while vasoconstriction is limited to peripheral portions and smaller branches. This picture is associated with true benign hypertension.

In the second group, early cases show arteriolar hypertonus in the retina. A few cases present with advanced retinopathy of malignant type, the vessels having the features of hyperplastic change and hypertonus. Many other cases, however, present a fundus picture in which fibrosis, hyperplasia, and hypertonus are found in different portions of the same retinal artery and its branches. Although these latter cases develop more rapidly than the elderly involutionary type, they do not run the course of malignant hypertension; the fundus appearances are associated with a severe degree of benign hypertension and the arteriosclerosis is of a reactive or secondary type.

The ophthalmoscopic classification is supported by evidence adduced from 
statistical and histological studies, providing a method of recording the ocular appearances directly in terms of general pathology.

This work has been carried out mainly at the Tennent Institute of Ophthalmology, University of Glasgow. I should like to thank Professor W. J. B. Riddell for his constant encouragement and for laboratory facilities during the past 10 years. I am indebted to my colleagues in the Departments of Medicine, Pathology, and Ophthalmology, in the Western Infirmary, the Royal Hospital for Sick Children, the Royal Maternity and Women's Hospital, the Southern General Hospital, and the Victoria Infirmary, Glasgow, for generous access to cases and helpful cooperation. I wish to thank Dr. R. L. Richards for his critical interest and help in the formative period; and also Dr. J. A. W. McCluskie and his staff for collaboration in the clinical application of the method. I am indebted to Mr. John Watt for the preparation of the histological material and for the photographic records throughout the work. I should like to thank Miss Anne Douglas for her careful and painstaking labours in constructing the composite retinal photographs. Finally, I am especially indebted to Mr. Gabriel Donald of the Department of Medical Illustration, not only for his excellent fundus drawings and his accurate and detailed observations by means of fundus sketches and diagrams, often made under difficult conditions in which no other method of recording would have succeeded, but also for his close collaboration with me in all aspects of the work, and for the many invaluable criticisms and suggestions he has contributed at all stages to these studies.

\section{REFERENCES}

AllbutT, C. (1915). " Diseases of the Arteries". Macmillan, London.

DUKE-ELDER,.S. (1940). “Text-Book of Ophthalmology", vol. 3, p. 2696. Kimpton, London.

LovatT Evans, C. (1941). "Starling's Principles of Human Physiology", 8th ed., p. 176. Churchill, London.

WAGENER, H. P., and KeITH, N. M. (1937). XV Concilium Ophthalmologicum, 1, 1.

WILSON, C., and BYROM, F. B. (1939). Lancet, 1, 136. 